\title{
NONSIMPLE, RIBBON FIBERED KNOTS
}

\author{
KATURA MIYAZAKI
}

\begin{abstract}
The connected sum of an arbitrary knot and its mirror image is a ribbon knot, however the converse is not necessarily true for all ribbon knots. We prove that the converse holds for any ribbon fibered knot which is a connected sum of iterated torus knots, knots with irreducible Alexander polynomials, or cables of such knots. This gives a practical method to detect nonribbon fibered knots. The proof uses a characterization of homotopically ribbon, fibered knots by their monodromies due to Casson and Gordon. We also study when cable fibered knots are ribbon and results which support the following conjecture.
\end{abstract}

Conjecture. If a $(p, q)$ cable of a fibered knot $k$ is ribbon where $p(>1)$ is the winding number of a cable in $S^{1} \times D^{2}$, then $q= \pm 1$ and $k$ is ribbon.

\section{INTRODUCTION}

Casson and Gordon [4] introduced the notion of "homotopically ribbon" for a knot in a homology sphere, which is an analog of "ribbon" for a knot in $S^{3}$, and proved that a fibered knot in a homology sphere is homotopically ribbon if and only if its closed monodromy extends over a handlebody. (A ribbon knot is homotopically ribbon.) This reduces the study of ribbon fibered knots to that of automorphisms of a handlebody. In this spirit Casson and Long [5] developed an algorithm to determine if a surface automorphism is nullcobordant, and found a new example of an algebraically slice but nonribbon fibered knot. (Also see Bonahon [3].) In this paper we study ribbon fibered knots in light of the theorem of Casson-Gordon, but by a method different from Casson and Long's. Our method is suitable for detecting nonsimple nonribbon fibered knots, while theirs is restricted to simple ones. (A knot is said to be simple if every incompressible torus in the knot exterior is boundary parallel.)

A closed monodromy $f$ of a ribbon fibered knot $K$ preserves a certain "pattern" $h$ (a union of 2-submanifolds) induced by a decomposition of the knot exterior of $K$. Now $f$ extends over a handlebody $H$. If $(H, h)$ admits a characteristic submanifold in the sense of Johannson, then it is kept invariant by the extension of $f$. We thoroughly study the position of the characteristic submanifold in $H$ to see the relation among the prime summands of $K$. Our working question is as follows:

Received by the editors June 20, 1991.

1991 Mathematics Subject Classification. Primary 57M25; Secondary 57N60.

Key words and phrases. Ribbon, homotopically ribbon, fibered knot, monodromy, Johannson's characteristic submanifold. 
Question 1. Suppose that $K_{1}, \ldots, K_{n}$ are prime fibered knots in homology spheres and $K_{1} \# \cdots \# K_{n}$ is homotopically ribbon. Under what circumstances can we pair $\{1, \ldots, n\}$ into $\left\{i_{s}, j_{s}\right\}_{s=1}^{m}$ (so that $2 m=n$ ) such that $K_{i_{s}} \cong$ $-K_{j_{s}}$ ?

Theorem 8.6. There is a class $\mathscr{K}$ of fibered knots in homotopy spheres giving an affirmative answer to Question 1 . The class $\mathscr{K}$ contains the iterated torus knots, any fibered knot with an irreducible Alexander polynomial, and any iterated cable about such a knot.

As a result of this theorem the following knots are algebraically sliced but not ribbon. (The algebraically-sliceness is verified by Livingston and Melvin [22] and Kawauchi [20], respectively.)

(1) Example 1 in $\$ 8.1$, the connected sum of four iterated torus knots

$$
(2,13 ; 2,3) \#(2,15) \#(2,-15 ; 2,-3) \#(2,-13) \text {. }
$$

(2) Example 2 in $\S 8.1$, a $(2,1)$ cable of a - amphicheiral fibered knot with an irreducible Alexander polynomial (e.g., the $(2,1)$ cable of the figure eight knot).

By assuming $K_{1} \cong \ldots \cong K_{n}$ Question 1 relates to the famous question: does the classical knot concordance group contain any nontrivial elements of finite order other than 2? Replacing the assumption of sliceness by homotopically ribbon, we ask:

Question 2. Let $K$ be a fibered knot in a homology sphere. If $n K$ ( $n$-fold connected sum of $K$ ) is homotopically ribbon, is $2 K$ homotopically ribbon?

Theorem 5.8. Question 2 has an affirmative answer if every slice knot in a homology sphere bounding a contractible 4-manifold is homotopically ribbon.

Let $J$ be a knot in $S^{1} \times D^{2}\left(\subset S^{3}\right)$, and $k$ a knot in a homology sphere $M$. Then $J(k)$ denotes the knot $f(J)$ in $M$ where $f: S^{1} \times D^{2} \rightarrow N(k)(\subset M)$ is the canonical framing of $k$ which sends the core of $S^{1} \times D^{2}$ to $k$ preserving orientations. The knot $J$ is said to be a $(p, q)$ cable of $S^{1} \times D^{2}$ if $J$ is a $p$ (longitude $)+q($ meridian $)$ curve on $\partial\left(S^{1} \times \frac{1}{2}\right)$.

Theorem 8.5. Let $K$ be a $(p, q)$ cable of a fibered knot $k$ in a homology sphere where $p>1$. If $n K$ is homotopically ribbon for some $n>0$, then (1) so is $K$, (2) $q= \pm 1$; $k$ is homotopically ribbon if $p>2$.

There is a fibered knot $k$ in a homology sphere such that $k$ is not homotopically ribbon, but the $(2,1)$ cable of $k$ is (Example 3 in $\S 8.1$ ), so that the assumption $p>2$ in (2) above is necessary. However, for a cable knot in a homotopy sphere the assumption $p>2$ can be omitted if 3-manifold groups are residually finite (Theorem 8.5 , part 3 ). It is easy to see a $(p, 1)$ cable of a homotopically ribbon fibered knot is homotopically ribbon. Thus, these results prove that only such a knot is a homotopically ribbon, cable fibered knot in a homotopy sphere if Thurston's geometrization conjecture is true.

Question 3. If a $(p, q)$ cable of a classical knot $k$ is ribbon (resp. slice) where $p>1$, is $k$ ribbon (resp. slice) and $q= \pm 1$ ?

This paper is organized as follows. Sections 1 through 4 are preliminary parts. Section 1 relates homotopically ribbon concordance and compression 
bodies. The precise definition of the monodromy of a fibered knot in a homotopy sphere with unknotted holes is given in $\S 2$. We determine the fibered knots with periodic monodromies also in $\S 2$. Given a fibered knot in a homology sphere, a torus decomposition of the knot exterior breaks the monodromy into periodic or irreducible automorphisms. In $\S 3$, we study the properties of each automorphism, which are used to characterize the action of a closed monodromy on a boundary pattern $h$ in $\S 5.1$. In $\S 4$, a "dual knot" $J^{*}$ is defined for certain fibered knots $J$ in a homotopy sphere with unknotted holes; then $J\left(J^{*}(0)\right)$ is homotopically ribbon. Sections 5 through 7 are the main parts of our work. Theorems 5.2-5.4, stated in $\S 5.1$, are the fundamental results, which are proved in $\S \S 5,6,7$ respectively. All results stated in the introduction are consequences of the fundamental theorems or the arguments used in the proofs. Section 8 deals with the specific cases, e.g., cable knots.

I would like to thank my supervisor Professor Cameron Gordon for helpful conversations and his encouragement. Thanks are also due to Professor Akio Kawauchi who informed me that the $(2,1)$ cable of the figure eight knot is algebraically slice, but possibly nonslice.

\section{HOMOTOPICALLY RIBBON CONCORDANCE}

All manifolds including knots and surfaces are suitably oriented and smooth. For a manifold $M,-M$ is obtained from $M$ by reversing its orientation. All diffeomorphisms are orientation preserving. For an automorphism $f$ of a manifold $X,-f$ denotes the automorphism $f$ on $-X$. The symbol $\cong$ means diffeomorphic. The symbol $\simeq$ means isotopic. Unlabeled homomorphisms are induced by inclusions unless otherwise stated.

Let $K_{i}, i=0,1$, be a knot in a homology 3-sphere $M_{i}$. We write $K_{0} \cong K_{1}$ if $\left(M_{0}, K_{0}\right) \cong\left(M_{1}, K_{1}\right)$. We denote by $K_{0} \# K_{1}$ the connected sum $\left(M_{0}, K_{0}\right) \#$ $\left(M_{1}, K_{1}\right)$. The definitions below are based on a homotopy property of ribbon concordance between classical knots [13, Lemma 3.1]. We shall write $\left(M_{1}, K_{1}\right) \geq\left(M_{0}, K_{0}\right)$ or simply $K_{1} \geq K_{0}$ if there are a 4-manifold $X^{4}$ with the homology groups of $S^{3}$ and an annulus $A$ in $X^{4}$ such that

$$
\begin{aligned}
& \left(\partial X^{4}, A \cap \partial X^{4}\right)=\left(M_{1}, K_{1}\right) \amalg\left(-M_{0},-K_{0}\right), \\
& \pi_{1}\left(M_{1}-K_{1}\right) \rightarrow \pi_{1}\left(X^{4}-A\right) \text { is surjective, and } \\
& \pi_{1}\left(M_{0}-K_{0}\right) \rightarrow \pi_{1}\left(X^{4}-A\right) \text { is injective. }
\end{aligned}
$$

We say $K_{1}$ is homotopically ribbon concordant to $K_{0}$. A knot $K_{1}$ is homotopically ribbon if $K_{1} \geq 0$, the unknot in $S^{3}$.

Let $K$ be a knot in a homology 3-sphere $M$. A knot $K$ is trivial if $K$ bounds a disk in $M$. A nontrivial knot $K$ is prime if $K$ cannot be written as $K_{0} \# K_{1}$ where neither of $K_{i}$ is a trivial knot in $S^{3}$. Let $F$ be a compact connected surface. We say that $K$ is a fibered knot in $M$ with fiber $F$ if there is an automorphism $f: F \rightarrow F$ (the monodromy of $K$ ) such that $\partial F \cong S^{1}$, $f \mid \partial F=\mathrm{id}$, and the mapping torus $F \times I / f(\equiv F \times[0,1] /((f(x), 0) \sim(x, 1)$ for all $x$ in $F)$ ) is diffeomorphic to $\overline{M-N(K)}$ where $x_{0} \times I / f, x_{0} \in \partial F$, is a meridian of $K$. Note that $\overline{M-N(K)}$ is a Haken manifold.

Lemma 1.1. Let $K_{1} \geq K_{2} \geq \cdots$ be a nonincreasing sequence of fibered knots in homology spheres. Then there is some $m$ such that $K_{n}=K_{m}$ for all $n \geq m$. 
Proof. Since the knot exterior of $K_{i}$ is Haken, Lemmas 3.2 and 3.4 in Gordon [13] hold. Thus there is some $m$ such that $\operatorname{deg} \Delta_{m}(t)=\operatorname{deg} \Delta_{n}(t)$ for all $n \geq$ $m$, where $\Delta_{i}(t)$ denotes the Alexander polynomial of $K_{i}$. The commutator subgroup of a fibered knot group is free and hence transfinitely nilpotent [13, p. 157], hence Lemma 3.4 shows that $K_{n}=K_{m}$ for all $n \geq m$.

A compression body $B$ is a cobordism rel $\partial$ between surfaces $\partial_{+} B$ and $\partial_{-} B$ such that $B \cong \partial_{+} B \times I \cup 2$-handles $\cup$ 3-handles, where handles are attached to $\partial_{+} B \times 1$, and $\partial_{-} B$ has no 2-sphere components. A compression body is trivial if $B=\partial_{+} B \times I$.

Lemma 1.2. Let $f, F, K, M$ be as above. Suppose that $f$ extends to an automorphism $\varphi$ of a compression body $B$ so that $\partial_{+} B=F, \partial_{-} B$ is connected and $\varphi \mid \partial F \times I=\mathrm{id}$. Then there is a fibered knot $K^{\prime}$ in a homology 3-sphere $M^{\prime}$ such that $\varphi \mid \partial_{-} B$ is its monodromy and $(M, K) \geq\left(M^{\prime}, K^{\prime}\right)$. Furthermore, $\Delta_{1}(t) \doteq \Delta_{0}(t) f(t) f\left(t^{-1}\right)$ for some $f(t) \in Z[t]$ where $\Delta_{1}, \Delta_{0}$ denote the Alexander polynomials of $K, K^{\prime}$ respectively.

Proof. Glue $S^{1} \times D^{2}$ to the mapping torus of $\varphi \mid \partial_{-} B: \partial_{-} B \rightarrow \partial_{-} B$ along their boundaries so that a simple loop $p \times I / \varphi$ is a meridian of $S^{1} \times D^{2}$ for all $p \in \partial\left(\partial_{-} B\right)$. The resulting manifold $M^{\prime}$ say, is a homology 3-sphere containing a fibered knot $S^{1} \times 0$ whose monodromy is $\varphi \mid \partial_{-} B: \partial_{-} B \rightarrow \partial_{-} B$. Let $K^{\prime}=$ $S^{1} \times 0 \subset M^{\prime}$. Glue $S^{1} \times D^{2} \times I$ to $B \times I / \varphi$ so that for all $t \in I$ and $q \in \partial F$, $S^{1} \times \partial D^{2} \times t=\partial F \times t \times I / \varphi$ and the simple loop $q \times t \times I / \varphi$ is a meridian of $S^{1} \times D^{2} \times t$. Call this 4-manifold $X^{4}$ and let $A=S^{1} \times 0 \times I \subset X^{4}$. Then

$$
\left(\partial X^{4}, \partial A\right)=(M, K) \cup\left(M^{\prime}, K^{\prime}\right) \text {. }
$$

Since $\pi_{1}\left(\partial_{+} B\right) \rightarrow \pi_{1}(B)$ is surjective and $\pi_{1}\left(\partial_{-} B\right) \rightarrow \pi_{1}(B)$ is injective, it follows that $\pi_{1}(M-K) \rightarrow \pi_{1}\left(X^{4}-A\right)$ is surjective and $\pi_{1}\left(M^{\prime}-K^{\prime}\right) \rightarrow$ $\pi_{1}\left(X^{4}-A\right)$ is injective.

The statement about $\Delta_{0}$ and $\Delta_{1}$ follows from the argument in the remark of [1, p. 541]. (Gilmer [10, Proposition 1.4] proves this inequality for ribbon concordance between classical knots.)

Lemma 1.3. Let $M, M^{\prime}$ be as in Lemma 1.2. Suppose that 3-manifold groups are residually finite. Then $\pi_{1}(M)=1$ implies $\pi_{1}\left(M^{\prime}\right)=1$.

Proof. Let $B$ be as in Lemma 1.2 and let $\partial_{-} B=F^{\prime}$; then $B=\left(F^{\prime} \times\right.$ I) $\cup{ }^{m} 1$-handles. Let $y_{1}, \ldots, y_{m}$ be elements of $\pi_{1}(B)$ corresponding to the 1-handles in $B$ and let $x_{1}, \ldots, x_{n}$ be free generators of $\pi_{1}\left(F^{\prime}\right)$, so that $\pi_{1}(B)=\left\langle x_{1}, \ldots, x_{n}, y_{1}, \ldots, y_{m}\right\rangle$. Let $\varphi_{*}$ denote the isomorphism of $\pi_{1}(B)$ induced by $\varphi$. Setting $s_{i}=t u_{i} t^{-1} \varphi_{*}\left(u_{i}\right)^{-1}$ where $u_{i}=x_{i}$ (for $1 \leq i \leq n$ ) or $=y_{i-n}($ for $n+1 \leq i \leq n+m)$, we then have

$$
\pi_{1}(B \times I / \varphi)=\left(\pi_{1}(B) *\langle t\rangle\right) / N\left(s_{1}, \ldots, s_{n+m}\right)
$$

where $N(\cdots)$ is normal closure. Note that $t$ corresponds to a loop $p \times I / \varphi$, $p \in \partial F^{\prime}$.

Defining $X^{4}$ as in the proof of Lemma 1.2, we have the epimorphism from $\pi_{1}(B \times I / \varphi)$ to $\pi_{1}\left(X^{4}\right)$ induced by the quotient map, and have the group presentation

$$
\pi_{1}\left(X^{4}\right)=\left(\left\langle x_{1}, \ldots, x_{n}\right\rangle *\left\langle y_{1}, \ldots, y_{m}\right\rangle\right) / N\left(\bar{s}_{1}, \ldots, \bar{s}_{n+m}\right)
$$


where $\bar{s}_{i}$ is the image of $s_{i}$. Since $\varphi\left(F^{\prime}\right)=F^{\prime}, \varphi_{*}\left(x_{i}\right)$ is a word in $\left\{x_{j}\right\}$, so

$$
\left\langle x_{1}, \ldots, x_{n}\right\rangle / N\left(\bar{s}_{1}, \ldots, \bar{s}_{n}\right) \cong \pi_{1}\left(M^{\prime}\right) \text {. }
$$

Let $G=\pi_{1}\left(M^{\prime}\right)$. We then have

$$
\pi_{1}\left(X^{4}\right)=\left(G *\left\langle y_{1}, \ldots, y_{m}\right\rangle\right) / N\left(\bar{s}_{n+1}, \ldots, \bar{s}_{n+m}\right) .
$$

Since $\pi_{1}(F) \rightarrow \pi_{1}(B)$ is surjective, the first row homomorphism in the commutative diagram below is surjective. (The vertical arrows are induced by the quotient maps.) Hence, the homomorphism $\pi_{1}(M) \rightarrow \pi_{1}\left(X^{4}\right)$ is also surjective. It follows from $\pi_{1}(M)=1$ that $\pi_{1}\left(X^{4}\right)=1$.

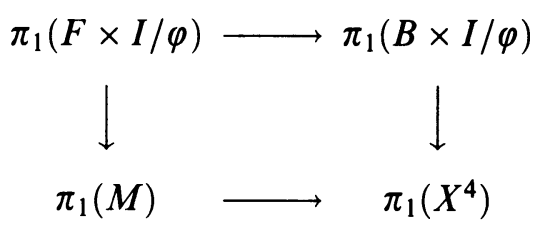

Let us show $G=1$. Since $H_{1}\left(M^{\prime}\right)$ is trivial, $\left\{\bar{s}_{n+i}\right\}_{i=1}^{m}$ is an independent system of equations in $\left\{y_{i}\right\}$. By our assumption $G$ is residually finite. Reference [9] proves that $G$ is a subgroup of $\pi_{1}\left(X^{4}\right)$, thus $G=1$.

Suppose that Thurston's geometrization conjecture holds. Then the Poincare conjecture holds and 3-manifold groups are residually finite. Then, by Lemmas 1.2 and 1.3 the compression of the monodromy (in the way described in 1.2) of a fibered knot in $S^{3}$ gives rise to a homotopically ribbon concordance between classical knots.

\section{FIBERED KNOTS IN HOMOTOPY SPHERES WITH UNKNOTTED HOLES}

For a homotopy sphere $P$ and a nonnegative integer $s$ let $P(s)$ denote the exterior of an $s$-component trivial (possibly empty) link, $\bigcup_{i=1}^{s} l_{i}$, in $P$. We call $P(s)$ a homotopy sphere with unknotted holes. Let $J$ be a simple loop in $P(s)$. We say that $J$ is a fibered knot in $P(s)$ with fiber $F$ if there is an automorphism $f$ of $F$ and a diffeomorphism $g:(F \times I) / f \rightarrow \overline{P(s)-N(J)}$ such that under this identification the following hold.

1. $(\partial F \times 0) \cap \partial N(J)$ is a preferred longitude of $J$ in $P$;

2. $(\partial F \times 0) \cap \partial P(s)$ consists of meridians of $\bigcup l_{i}$ in $P$.

Condition 2 shows that the fibration of $\overline{P(s)-N(J)}$ extends over $\overline{P-N(J)}$, i.e. we can regard $J$ as a fibered knot in $P$. Let $\partial_{*} F$ be the components of $\partial F$ such that $\partial_{*} F \times 0=(\partial F \times 0) \cap N(J)$. Note that the definition of $\partial_{*} F$ depends on $J$. The automorphism $f$ acts on the set of components of $\partial F-\partial_{*} F$ and divide it into orbits. The number of orbits is $s(=|\partial P(s)|)$. Let $\partial_{1} F, \ldots, \partial_{s} F$ be representatives of the orbits and let $m(i)$ be the cardinality of the orbit containing $\partial_{i} F, 1 \leq i \leq s$. We say that $f:\left(F, \partial_{*} F\right) \rightarrow\left(F, \partial_{*} F\right)$ is the monodromy of $J$ if the following hold.

1. $f \mid \partial_{*} F=\mathrm{id}$, and $p \times I / f$ is a meridian of $J$, where $p \in \partial_{*} F$;

2. for $1 \leq i \leq s f^{m(i)} \mid \partial_{i} F=\mathrm{id}$ and the simple loop $\left(\bigcup_{j=1}^{m(i)} f^{j}\left(p_{i}\right) \times I\right) / f$ is a preferred longitude of $l_{i}$ where $p_{i} \in \partial_{i} F$. 
Remark. If $J$ is a fibered knot in $P(s)=\overline{P-\bigcup_{i=1}^{s} N\left(l_{i}\right)}$, then in the sense of Eisenbud and Neumann [8] the multilink $\left(P, 1 J \cup 0 l_{1} \cup \cdots \cup 0 l_{s}\right)$ is fibered. Hence, as is pointed out in [8, p. 34], the fibration is unique up to isotopy. Similarly a fibration for a fibered knot in a homology sphere is unique up to isotopy.

Let $J_{i}, i=0,1$, be fibered knots in homotopy spheres with unknotted holes $M_{i}$. We write $J_{0} \cong J_{1}$ as fibered knots if there is a diffeomorphism from $\left(M_{0}, J_{0}\right)$ to $\left(M_{1}, J_{1}\right)$ which preserves the fibrations. The knot $-J_{i}$ denotes the knot $\left(-M_{i},-J_{i}\right)$.

Lemma 2.1. Let $f_{i}: F_{i} \rightarrow F_{i}$ be the monodromy of a fibered knot $J_{i}$ in a homotopy sphere with unknotted holes $M_{i}, i=0,1$. Suppose that $f_{0} \amalg-f_{1}$ extends to an automorphism $\varphi$ of a 3-manifold $U$ such that

1. $U \cong F_{0} \times I$; this diffeomorphism restricts to $F_{0} \stackrel{\mathrm{id}^{\prime}}{\longrightarrow} F_{0} \times 0$ and $-F_{1} \cong$ $F_{0} \times 1$,

2. $\varphi$ is the identity on $\partial_{*} F_{0} \times I$, and

3. $\partial_{*} F_{0} \times 1=\partial_{*} F_{1}$.

Then $J_{0} \cong J_{1}$ as fibered knots.

Proof. The natural projection from $F_{0} \times 0$ to $-\left(F_{0} \times 1\right)$ induces a diffeomorphism $g: F_{0} \rightarrow F_{1}$ such that $g\left(\partial_{*} F_{0}\right)=\partial_{*} F_{1}$ and $g f_{0} g^{-1} \simeq f_{1} \operatorname{rel} \partial_{*} F_{1}$ [26, Lemma 3.5]. By condition 1 of the definition of monodromy, $g$ induces a diffeomorphism from $\overline{M_{0}-N\left(J_{0}\right)}$ to $\overline{M_{1}-N\left(J_{1}\right)}$ carrying a meridian of $J_{0}$ to that of $J_{1}$. It follows that $J_{0} \cong J_{1}$ as fibered knots.

Lemma 2.2. Let $f_{i}: F_{i} \rightarrow F_{i}, J_{i}, M_{i}, i=0,1$, be as in Lemma 2.1. Let $b$ be a component of $\partial F_{0}-\partial_{*} F_{0}$ and $m$ be the smallest positive integer such that $f_{0}^{m}(b)=b$. Suppose that $f_{0} \amalg-f_{1}$ extends to an automorphism $\varphi$ of $a$ 3-manifold $U$ which satisfies conditions 1 and 2 of Lemma 2.1. Then, $\varphi^{m}$ can be isotoped rel $F_{0} \times\{0,1\}$ so that $\varphi^{m}$ is the identity on $\left(\partial_{*} F_{0} \cup b\right) \times I$.

Proof. Let $x_{0}$ be a point on $b$. By the definition of monodromy $\varphi^{m}$ fixes $x_{0} \times\{0,1\}\left(\subset F_{0} \times I\right)$. To prove the lemma it suffices to show that $\varphi^{m}\left(x_{0} \times I\right)$ is rel $x_{0} \times\{0,1\}$ isotopic to $x_{0} \times I$ in $b \times I$. Let $n$ be the winding number of $\varphi^{m}\left(x_{0} \times I\right)$ rel $x_{0} \times \partial I$ in the annulus $b \times I$. We shall show $n=0$. The natural projection from $F_{0} \times 0$ to $-\left(F_{1} \times 0\right)$ induces a diffeomorphism $g: \overline{M_{0}-N\left(J_{0}\right)}$ to $\overline{M_{1}-N\left(J_{1}\right)}$. Let $T_{0}, T_{1}$ be the components of $\overline{M_{i}-N\left(J_{i}\right)}, i=0,1$, containing $b, g(b)$ respectively. Let $\lambda_{i}, \mu_{i}$ be a longitude-meridian system on $T_{i}$ determined by the trivial link removed from a homotopy sphere. We first assume $g\left(\partial N\left(J_{0}\right)\right)=\partial N\left(J_{1}\right)$, i.e., condition 3 of Lemma 2.1 holds. Then $g$ sends a meridian of $J_{0}$ to that of $J_{1}$, and hence $g$ extends to a diffeomorphism from $M_{0}$ to $M_{1}$. On the other hand $g$ carries $\lambda_{0}$, a longitude of $T_{0}$, to a simple loop $\lambda_{1}+n \mu_{1}$ in $T_{1}$. Since $\lambda_{i}$ is null-homologous (in fact, nullhomotopic) in $M_{i}$, we obtain $n\left[\mu_{1}\right]=\left[\lambda_{1}+n \mu_{1}\right]=\left[g\left(\lambda_{0}\right)\right]=0 \in H_{1}\left(M_{1}\right)$. It follows that $n=0$, proving the lemma.

Next assume $T=g\left(\partial N\left(J_{0}\right)\right) \neq \partial N\left(J_{0}\right)$. Then $g$ sends a meridian of $J_{0}$ to a longitude of $T$, say $\lambda$. Glue $S^{1} \times D^{2}$ to $\overline{M_{1}-N\left(J_{1}\right)}$ along $T$ so that $\lambda$ is null-homotopic in $S^{1} \times D^{2}$. Let $M$ be the resulting manifold; $g$ extends to a diffeomorphism from $M_{0}$ to $M$. Then $\left[\lambda_{1}+n \mu_{1}\right]=\left[g\left(\lambda_{0}\right)\right]=0 \in H_{1}(M)$. We claim that $\lambda_{1}$ is null-homologous in $M$, establishing Lemma 2.2 as above. In 


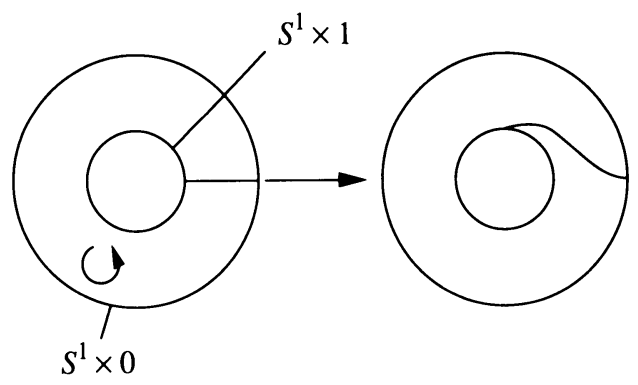

Figure 1

$\overline{M_{1}-N\left(J_{1}\right)}$ the longitude $\lambda_{1}$ of $T_{1}$ is homologous to a multiple of a meridian of $J_{1}$. However, since the fiber $F_{1}$ meets $T$ in a single loop, a meridian of $J_{1}$ is homologous to $\lambda$ in $\overline{M_{1}-N\left(J_{1}\right)}$. Hence the claim follows from the fact that $\lambda$ is null-homologous in $M$.

In the rest of this section we shall describe periodic monodromies of fibered knots in homotopy spheres with unknotted holes. In fact, these monodromies turn out to be those of torus knots in $S^{1} \times D^{2}$ or $S^{3}$.

Let $g$ be a periodic automorphism of a compact surface $G$, and let $x \in$ $G$ be a fixed point of $g$. Then there are a neighborhood $B$ of $x$ and a diffeomorphism $h$ from $B$ to the unit disk of $R^{2}$ such that $h(0)=0$ and $h g h^{-1}$ is the rotation of angle $\theta$ around 0 . Define a fixed point data of $x$ to be $\theta / 2 \pi$, which is determined up to addition of integers. Thus we consider that a fixed point data takes its value in $Q / Z$. [2] or [7] shows that if $G$ is a closed surface and the set of fixed point data of $g^{n}$ for all $n$ are paired to cancel each other, then $g \mid G: G \rightarrow G$ is periodically null-cobordant. However, our monodromies are not necessarily defined on closed surfaces. We need the notion of an $r$-Dehn twist for a rational number $r$ as follows.

Let $A$ be an annulus with $\partial A=\alpha_{0} \cup \alpha_{1}$. An automorphism $\varphi$ of $A$ is an $r$-Dehn twist from $\alpha_{0}$ to $\alpha_{1}$ if there is a diffeomorphism $h: S^{1} \times I \rightarrow A$ such that (see Figure 1):

1. $h\left(S^{1} \times i\right)=\alpha_{i}$ for $i=0,1$;

2. the orientation of $S^{1} \times 0$ induced from $S^{1} \times I$ is counterclockwise;

3. $h \varphi h^{-1}(\theta, t)=(\theta+r t, t)$.

Lemma 2.3. Let $f: F \rightarrow F$ be the nontrivial periodic monodromy of a fibered knot $J$ in $P(s)$, where $P$ is a homotopy sphere and $s \geq 0$. Then assertion 1 or 2 below holds for some relatively prime $p>1$ and $q$.

1. $J$ is the $(p, q)$ torus knot in $S^{3}$ with genus of $F=(p-1)(q-1) / 2$ and $|\partial F|=1$.

2. $J$ is the $(p, q)$ cable of $S^{1} \times D^{2}\left(\subset S^{3}\right)$ with genus of.

$$
F=(p-1)(q-1) / 2 \text { and }|\partial F|=p+1 \text {. }
$$

Proof. Let $M=\overline{P(s)-N(J)}$. Recall that (1) $M=F \times I / f$ is a Seifert fibered manifold each of whose fiber is $\left(\bigcup_{k} f^{k}(x) \times I\right) / f$ for some $x \in F$, and (2) a meridian $\mu$ of $J$ in $\partial N(J)$ meets $F$ in a single point $\left(\in \partial_{*} F\right)$. By (1) the Seifert fibration of $M$ extends over $\overline{P-N(J)}$. If $\mu$ is isotopic in $\partial N(J)$ 
to a fiber of the fibration of $M$, then $f$ has a fixed point in $\partial F$. Therefore $f=$ id, a contradiction. The Seifert fibration of $M$ thus extends over $P(s)$, and hence over $P$. It follows that $P=S^{3}, s=0$ or 1 , and $J$ is a fiber of some Seifert fibration of $S^{3}$. This completes the proof.

Investigating the intersections of $F$ and a singular fiber of $S^{3}$ and those of $\partial F$ and a regular fiber of $S^{3}$, we obtain the following results.

Lemma 2.4. Let $f: F \rightarrow F$ be the monodromy of the $(p, q)$ torus knot in $S^{3}$ in part 1 or the $(p, q)$ cable of $S^{1} \times D^{2}$ in part 2 , where $p>1$.

1. Take a collar neighborhood $N$ of $\partial F$. Then after an isotopy $\operatorname{rel} \partial F f$ satisfies the following.

(a) $f(N)=N$ and $f \mid F-N$ is a periodic map of order $|p q| ; f^{k} \mid F-N$ has a fixed point only if $k$ is a multiple of $p$ or $q$.

(b) $f \mid N$ is a $1 / p q$-Dehn twist from $\partial F$ to $\partial N-F$.

(c) $f^{p} \mid F-N$ has $p$ fixed points of data $p / q(\in Q / Z)$.

(d) $f^{q} \mid F-N$ has $|q|$ fixed points of data $q / p$.

2. Take pairwise disjoint collar neighborhoods $N_{0}, \ldots, N_{p}$ of $\partial F$ such that $N_{0} \supset \partial_{*} F$. Then after an isotopy rel $\partial F f$ satisfies the following.

(a) $f\left(\bigcup N_{i}\right)=\bigcup N_{i}$ and $f \mid F-\bigcup N_{i}$ is a periodic map of order $|p q| ; f^{k} \mid F-$ $\cup N_{i}$ has a fixed point only if $k$ is a multiple of $p$.

(b) $f \mid N_{0}$ is a $1 / p q$-Dehn twist from $\partial_{*} F$ to $\partial N_{0}-\partial_{*} F$.

(c) $f^{q} \mid F-\bigcup N_{i}$ has $|q|$ fixed points of data $q / p$.

(d) $f^{p} \mid N_{i}$ is $a-p / q$-Dehn twist from $\partial N_{i} \cap \partial F$ to $\partial N_{i}-\partial F$ for $1 \leq i \leq p$.

(e) $f$ permutes $\partial F-\partial_{*} F$ cyclically.

Lemma 2.5. Let $f: F \rightarrow F$ be the monodromy of a torus knot or a cable of $S^{1} \times D^{2}$. Let $c$ be the component of $\partial F$ invariant under $f$. Denote $F \cup_{c=\partial} D^{2}$ by $G$. Then the automorphism $g=f \cup \mathrm{id}$ of $G$ is isotopic to an irreducible periodic map.

Proof. It suffices to check that $g$ is irreducible. After an isotopy $F / f$ is a disk with at most two cone points or an annulus with at most 1 cone point as an orbifold. Thus, $g$ can be isotoped so that $G / g$ is a sphere with at most 3 cone points or a disk with at most 2 cone points. Hence, $g$ is irreducible.

\section{DECOMPOSING MONODROMY OF FIBERED KNOTS}

Let $f: F \rightarrow F$ be the monodromy of a fibered knot $K$ in a homology sphere $M$. Let $\mathscr{T}$ be a minimal (possibly empty) set of incompressible tori in $X=$ $\overline{M-N(K)}$ which decomposes $X$ into a Seifert fibered manifold and a simple manifold. By Jaco and Shalen [18] or Johannson [19], $\bigcup \mathscr{T}$ is determined up to isotopy. Isotop $\bigcup \mathscr{T}$ so that $|F \cap \bigcup \mathscr{T}|$ is minimal and let $C=F \cap \bigcup \mathscr{T}$, a set of essential simple loops in $F$. By a homological argument we see that $C$ consists of separating loops in $F$. Thus for the closure of any component of $F-C, S$ say, exactly one component of $\partial S$ is equal to $\partial F$, or separates $\partial F$ from the other components of $\partial S$. We call this component the root component of $\partial S$, and denote it by $\partial_{r} S$. Let us break up $f$ into simpler monodromies by using $C$ in $F$. 
Lemma 3.1. Let $\mathscr{S}$ denote the set of closures of components of $F-C$. After an isotopy rel $\partial F$ of $f$ the monodromy $f$ satisfies the following.

1. $f(C)=C$.

2. Let $S \in \mathscr{S}$ such that $\partial S \supset \partial F$. If $K$ is a connected sum of $n$ prime knots with $n \geq 2$, then $S$ is a disk with $n$ punctures and $f \mid S=\mathrm{id}$.

For $S \in \mathscr{S}$ let $f_{S}$ denote the automorphism $f^{m} \mid S$ of $S$ where $m$ is the least positive integer such that $f^{m}(S)=S$. Call $f_{S}$ the adjoint automorphism of $S$. The lemmas below describe the properties of $f_{S}$.

Lemma 3.2. With the data of Lemma $3.1 f$ satisfies the following.

3. For any $S \in \mathscr{S} f_{S}$ is irreducible or isotopic to a periodic map.

4. Take $R,(\neq) S \in \mathscr{S}$. If $f^{k}$ preserves $R \cup S$ for some $k>0$ and $f^{k} \mid R \cup S$ is isotopic to a periodic map on $R \cup S$, then $R \cap S=\varnothing$.

Proof of Lemma 3.1. Under the natural identification $\overline{M-N(K \cup F)} \cong F \times$ $I$ the set of annuli $\overline{U \mathscr{T}-N(F)}$ can be isotoped to $C \times I$ because of the minimality of $|F \cap \bigcup \mathscr{T}|$. This completes part 1 .

Since $K$ is a connected sum of $n$ prime knots, there are $n-1$ pairwise nonparallel, separating, essential annuli $A_{1}, \ldots, A_{n-1}$ in $\overline{M-N(K)}=X$ such that each component of $\partial A_{i}$ is a meridian of $K$. Let $C_{n}$ be the regular neighborhood of $\partial X \cup\left(\bigcup_{i=1}^{n-1} A_{i}\right)$ in $X$; then $C_{n} \cong$ (a disk with $n$ punctures) $\times S^{1}$ is an essential Seifert fibered space in $(X, \partial X)$ in the sense of [19]. By $[19,10.10]\left(C_{n}, \partial X\right)$ can be isotoped into the characteristic submanifold of $(X, \partial X)$. Now $S \times I / f=N$ say, is the closure of the component of $X-$ $\bigcup \mathscr{T}$ containing $\partial X$. We can regard $(N, \partial X)$ as a Seifert fibered manifold component of the characteristic submanifold of $(X, \partial X)$, so that $C_{n} \subset N$ after an isotopy rel $\partial X$. Isotop the incompressible tori $\partial C_{n}-\partial X$ in $N$ so as to be saturated by the fibration of $N$. Since $C_{n}$ has a unique Seifert fibration up to isotopy, the Seifert fibration of $N$ is an extension of that of $C_{n}$. With respect to the fibration of $C_{n}, \partial S \cap \partial X$ (a preferred longitude of $K$ ) meets each $S^{1}$-fiber on $\partial X$ in a single point. Therefore each $S^{1}$-fiber of $N$ meets $S$ in a single point, so that $f \mid S \simeq$ id $\operatorname{rel} \partial F$. Since $M$ does not contain nonseparating tori, $S$ is a punctured disk. If $S$ is a disk with more than $n$ punctures, then $K$ is a connected sum of more than $n$ nontrivial knots, a contradiction. Hence, $S$ is a disk with $n$ punctures.

Proof of Lemma 3.2. If $f_{S}$ is reducible, then $S \times I / f_{S}$ (the closure of a component of $X-\cup \mathscr{T}$ ) contains an essential torus, and hence is a Seifert fibered manifold. Thus $f_{S}$ is isotopic to a periodic map, completing part 3. Suppose that part 4 is false. Without loss of generality assume $R \cup S=\partial_{r} S$. Then $T=\partial_{r} S \times I / f_{R}$ is an element of $\mathscr{T}$, and by the assumption of part $4\left(R \times I / f_{R}\right) \cup_{T}\left(S \times I / f_{S}\right)$ is a boundary irreducible Seifert manifold in $X$. Thus, $\bigcup \mathscr{T}-T$ divides $X$ into a Seifert fibered manifold and a simple manifold, contradicting the minimality of $\mathscr{T}$.

If $K$ is a fibered knot in a homotopy sphere, then we can obtain more information about $f_{S}$.

Lemma 3.3. With the data of Lemma 3.2 assume that $M$ is a homotopy 3sphere. Then after an isotopy rel $\partial F$ satisfies the following (in addition to 1-4 above). 
5. For any $S \in \mathscr{S} f_{S}$ is either the identity on a punctured disk, or irreducible and not isotopic to the identity.

6. For any $S \in \mathscr{S} f_{S}:\left(S, \partial_{r} S\right) \rightarrow\left(S, \partial_{r} S\right)$ is the monodromy of a fibered knot in a homotopy sphere with unknotted holes $Y$, (so that $\partial_{r} S=\partial_{*} S$ ). Moreover, if $M=S^{3}$, then $Y$ is the exterior of a trivial link in $S^{3}$.

Proof. Let $N=S \times I / f_{S}$, the closure of a component of $M-\cup \mathscr{T}$. Let $T_{0}=\partial_{r} S \times I / f_{S}, T_{1}, \ldots, T_{s}$ be the boundary components of $\partial N$ and let $E_{i}$, $0 \leq i \leq s$, be the closures of components of $M-T_{i}$ such that $E_{i} \cap \operatorname{Int} N=\varnothing$. Then $E_{0}$ is a homotopy solid torus containing $K$, and for $i>0, E_{i}$ is the exterior of a nontrivial fibered knot in a homotopy sphere. Let $\mu$ be a simple loop on $T_{0}$ null-homotopic in $E_{0}$. By a homological argument $\mu$ meets $\partial_{r} S$ in a single point (after an isotopy in $T_{0}$ ).

Proof of part 6. Glue $S^{1} \times D^{2}$ to $N$ along $T_{0}$ so that $\mu$ is null-homotopic in $S^{1} \times D^{2}$. Then each $T_{i}$ is compressible in $N \cup S^{1} \times D^{2}$. Let $N^{\prime}=N \cup S^{1} \times D^{2}$ and $K^{\prime}=S^{1} \times 0 \subset N^{\prime}$. There are simple loops $c_{i} \subset T_{i}, 1 \leq i \leq s$, bounding disks in $N^{\prime}$. By a cut-and-paste argument these disks can be taken to be pairwise disjoint. Then gluing solid tori $\left(S^{1} \times D^{2}\right)_{i}, 1 \leq i \leq s$, to $N^{\prime}$ along $\partial N^{\prime}$ so that each component of $\partial S-\partial_{r} S$ bounds a disk in some $\left(S^{1} \times D^{2}\right)_{i}$, we obtain a homotopy sphere $M^{\prime}$, say. By a homological argument $c_{i}$ is a longitude of $\left(S^{1} \times D^{2}\right)_{i}$, so $\bigcup_{i=1}^{s}\left(S^{1} \times 0\right)_{i}$ is a trivial link in $M^{\prime}$. It follows that $K^{\prime}$ is a fibered knot in $M^{\prime}(s)=N^{\prime}$ with $\partial_{*} S=\partial_{r} S$. If $M=S^{3}$, then $E_{0}$ and $\overline{M-E_{i}}, 1 \leq i \leq s$, are solid tori. Replacing $E_{1}$ by a solid torus in the manner above, we still have $S^{3}$. Thus induction on $s$ proves $M^{\prime} \cong S^{3}$.

Now we show that $f$ can be isotoped so that $f_{S}$ is the monodromy of $K^{\prime}$. Let $R \in \mathscr{S}$ be adjacent to $S$ with $R \cap S=\partial_{r} S$. Assume that $f_{R}$ is the monodromy of the fibered knot $\left(Y, K^{\prime \prime}\right)$ (say) which is obtained from $R \times I / f_{R}$ in the same say as $\left(M^{\prime}(s), K^{\prime}\right)$ from $S \times I / f_{S}$. Then by condition 2 of the definition of monodromy the simple loop $\mu^{\prime}=\left(\bigcup_{j}\left(f_{R}\right)^{j}(p) \times I\right) / f_{R}$, where $p \in \partial_{r} S$, is null-homotopic in $Y$, and hence in $E_{0}$. Since $\mu^{\prime}=p \times I / f_{S}, \mu^{\prime}$ is a meridian of $K^{\prime}$ in $M^{\prime}(s)$. This implies that $f_{S}$ satisfies condition 1 of the definition of monodromy. An isotopy of $f_{S}$ in a neighborhood of $\partial S-\partial_{r} S$ can deform $f_{S}$ to be the monodromy of $K^{\prime}$ of $M^{\prime}(s)$. Let $m$ be the least positive integer such that $f^{m} \mid S=f_{S}$. Since $f^{k}(S) \cap S=\varnothing$ for $1 \leq k<m$, the isotopy of $f_{S}$ can be induced by an isotopy of $f$ in a neighborhood of $\partial S-\partial_{r} S$ in $F$. Thus part 6 of Lemma 3.3 is proved by deforming $f$ on the closure of each component of $F-C$ beginning with the component containing $\partial F$.

Proof of part 5. If $f_{S} \simeq$ id, then $S$ is a punctured disk. By part 6 any simple loop $p \times I / f_{S}, p \in \partial S$, is null-homotopic in $N^{\prime}$, the exterior of a trivial link in $S^{3}$. Hence each such a loop is an $S^{1}$-fiber of $N \cong S \times S^{1}$, so that $f_{S} \simeq \operatorname{id}$ rel $\partial S$. If $f_{S}$ is reducible, then $N$ is a boundary irreducible Seifert fibered submanifold in $\overline{M-N(K)}$ by part 3 of Lemma 3.2. Since $N$ contains an essential torus, by the proof of Lemma 2.1 in Swarup [24] $N$ is diffeomorphic to (a punctured disk) $\times S^{1}$ such that the simple loop $\mu$ is an $S^{1}$-fiber. Since $\left|\mu \cap \partial_{r} S\right|=1$, each $S^{1}$-fiber of $N$ meets $S$ in a single point, so that $f_{S} \simeq$ id. Therefore, we can isotop $f$ so that parts 5 and 6 hold. 
Suppose $J$ is a fibered knot in $P(n)$ where $P$ is a homotopy sphere and $n>$ 0 , and $k_{i}$ is a fibered knot in a homology sphere $M_{i}, 1 \leq i \leq n$. Numbering the components of $\partial P(n)$ from 1 to $n$, we can uniquely define the fibered knot $J\left(k_{1}, \ldots, k_{n}\right)$ as follows. Let $M$ be the homology sphere obtained from $P(n)$ by gluing each $\overline{M_{i}-N\left(k_{i}\right)}$ to the $i$ th component of $\partial P(n)$ so that longitudemeridian pairs of $\bigcup k_{i}$ correspond to meridian-longitude pairs of the solid tori $\overline{P-P(n)}$. Then let $J\left(k_{1}, \ldots, k_{n}\right)$ be the knot $J$ in $M$.

Conversely, we define a satellite presentation for a fibered knot $K$ in a homotopy sphere $M$ as follows. Let $T_{1}, \ldots, T_{n}$ be incompressible tori in $\overline{M-N(K)}$ such that no $T_{i}$ separates $T_{j}(i \neq j)$ from $\partial N(K)$ in $M$. By the proof of Lemma $3.3 \cup T_{i}$ divides $M$ into $M^{\prime}(n)$ containing $K$ where $M^{\prime}$ is a homotopy sphere, and the disjoint union of the exterior of some nontrivial fibered knots $k_{i}, 1 \leq i \leq n$, in homotopy spheres. Let $J$ denote the fibered knot $K$ in $M^{\prime}(n)$; then we write $K=J\left(k_{1}, \ldots, k_{n}\right)$, a satellite presentation of $K$. Note that this presentation depends on the choice of incompressible tori and the numbering of the chosen tori.

\section{4. $J$ AND $J^{*}$}

Let $J_{i}, i=0,1$, be fibered knots with fibers $F_{i}$ in homotopy spheres with unknotted holes. Let $f_{i}: F_{i} \rightarrow F_{i}$ be the monodromy of $J_{i}$. We write $J_{1}=J_{0}^{*}$ if there is a diffeomorphism $g: F_{0} \rightarrow-F_{1}$ such that $g\left(\partial_{*} F_{0}\right) \neq \partial_{*} F_{1}$ and $g f_{0} g^{-1} \simeq-f_{1}$ rel $\partial_{*} F_{1}$. In general, $J_{0}^{*}$ is not uniquely determined by $J_{0}$. But if $J_{0}^{*}$ exists, some component, say $b$, of $\partial F_{0}-\partial_{*} F_{0}$ is fixed by $f_{0}$ such that $f_{0}(b)=b$ and $J_{0}^{*}$ is equivalent to the fibered knot with monodromy $f_{0}:\left(F_{0}, b\right) \rightarrow\left(F_{0}, b\right)$. Hence, when $f_{0}$ leaves at most one component of $\partial F_{0}-\partial_{*} F_{0}$ invariant (e.g., $J_{0} \subset S^{1} \times D^{2}$ ), $J_{0}^{*}$ is uniquely determined if it exists. If $J_{1}=J_{0}^{*}$, then $f_{0}$ leaves $g\left(\partial_{*} F_{0}\right)$ invariant, so Lemma 2.2 shows $g f_{0} g^{-1} \simeq-f_{1} \operatorname{rel} \partial_{*} F_{1} \cup g\left(\partial_{*} F_{0}\right)$. Hence $J_{0} \cong J_{1}^{*}$.

If $J$ is a fibered knot in $S^{1} \times D^{2}$ intersecting some meridional disk once, then $J^{*} \cong-J$. For a nontrivial example of a pair of $J$ and $J^{*}$ see [10].

Proposition 4.1. Let $J$ be a fibered knot in a homotopy solid torus $V$. If $J^{*}$ exists, then the following hold.

1. $J$ represents a generator of $H_{1}(V)$.

2. Let $K=J_{1}\left(J\left(J^{*}(k)\right)\right)$, where $J_{1}, k$ are arbitrary fibered knots in a homotopy solid torus, a homology sphere, respectively. Then $K \geq J_{1}(k)$. In particular $J\left(J^{*}(0)\right) \geq 0$, where 0 is the unknot is $S^{3}$.

Proof. Let $F_{0}$ (resp. $F_{1}$ ) be the fiber of $J$ (resp. $J^{*}$ ) and let $f_{0}: F_{0} \rightarrow F_{0}$ (resp. $f_{1}: F_{1} \rightarrow F_{1}$ ) be the monodromy of $J$ (resp. $J^{*}$ ). Let $g: F_{0} \rightarrow-F_{1}$ be a diffeomorphism used to define $J^{*}$. Since the ambient space of $J$ has only one boundary component, $f_{0}$ acts on the components of $\partial F_{0}-\partial_{*} F_{0}$ cyclically. Since $f_{0}$ leaves $g^{-1}\left(\partial_{*} F_{1}\right)$ invariant, $\partial F_{0}-\partial_{*} F_{0}$ has just one component, proving part 1. By the definition of $J^{*}$ and Lemma 2.2 the automorphism $f_{0} \amalg f_{1}$ of $F_{0} \amalg F_{1}$ extends to an automorphism $\varphi$ of $F_{1} \times I$ such that $\varphi \mid F_{1} \times 1=$ $f_{1}, \varphi\left|-\left(F_{1} \times 0\right)=g f_{0} g^{-1}, \varphi\right|\left(\partial_{*} F_{1} \cup g\left(\partial_{*} F_{0}\right)\right) \times I=$ id. Let $F$ be the fiber of $J_{1}(k)$ with monodromy $f$. Take a separating annulus $N$ on $F$ such that after an isotopy $f \mid N=$ id and $f \mid \overline{F-N}$ breaks up into the monodromies of $k$ and $J_{1}$. Glue $F_{1} \times I$ to $F \times I$ by identifying $g\left(\partial_{*} F_{0}\right) \times I$ and $N$, and we get 


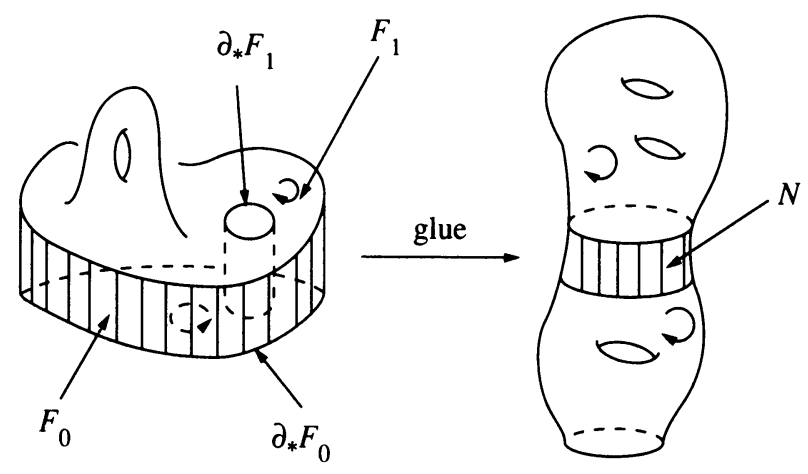

FIGURE 2

a compression body $B$ (Figure 2). Then $\psi=\varphi \cup f \times$ id is an automorphism of $B$ and $\psi \mid \partial_{+} B$ (resp. $\psi \mid \partial_{-} B$ ) is the monodromy of $K$ (resp. $J_{1}(k)$ ). Part 2 follows from Lemma 1.2.

Reference [10] defines $J^{*}$ for certain (possibly nonfibered) knots in $S^{1} \times D^{2}$, and proves that $J\left(J^{*}(0)\right)$ is slice. It also shows that $J\left(J^{*}\right) \subset S^{1} \times D^{2} \times 1$ and the core of $S^{1} \times D^{2} \times 0$ bound an annulus in $S^{1} \times D^{2} \times I$ with no local maxima. Hence, if $k$ is a knot in $S^{3}$, then $J_{1}\left(J\left(J^{*}(k)\right)\right)$ is ribbon concordant to $J(k)$, and in particular $J\left(J^{*}(0)\right)$ is a ribbon knot.

Given a homotopically ribbon, fibered knot, its closed monodromy extends over a handlebody $H$. In $\S 5.1$, by using a torus decomposition of the knot exterior a boundary pattern $h$ is defined for $H$. Then, the fundamental results Theorems 5.2-5.4 are stated in terms of $(H, h)$. Lemma 5.10 reduces the proofs of the theorems to the case when $h$ is a useful boundary pattern in the sense of Johannson [19]; then $(H, h)$ has a characteristic submanifold. In $\S \S 5.2,5.3$ we shall study the position of a characteristic submanifold for $(H, h)$ in the handlebody $H$, and prove Theorem 5.2. Sections 6 and 7 are devoted to the proofs of Theorems 5.3 and 5.4, respectively.

5.1. Fundamental theorems. Let $K_{i}, 1 \leq i \leq n$, be prime fibered knots in homology spheres with fibers $F_{i}$. Let $K$ be the fibered knot $K_{1} \# \ldots \# K_{n}$ in a homology sphere $M$ with monodromy $f: F \rightarrow F$. Suppose that $K$ is homotopically ribbon. As in $\S 3$ define a set of simple loops, $C$, in $F$ via a torus decomposition of $\overline{M-N(K)}$ and isotop $f$ rel $\partial F$ so as to satisfy Lemmas 3.1, 3.2 (and 3.3 if $M$ is a homotopy sphere). If $n \geq 2$, by Lemma 3.1, part 2 the closure of the component of $F-C$ containing $\partial F$ is a disk with $n$ punctures, say $D_{n}$, and $f \mid D_{n}=$ id. The components of $\overline{F-D_{n}}$ can be identified with the fibers $F_{1}, \ldots, F_{n}$. Define $\widehat{F}$ to be the closed surface $F \cup_{\partial} D^{2}$ and $\hat{f}=f \cup i d$, which is called the closed monodromy of $K$.

We define $h_{K}$, a set of 2-submanifolds in $\widehat{F}$, as follows. Define $B_{h_{K}}$ (or simply $B_{h}$ ) to be the closure of component of $\widehat{F}-C$ containing $\partial F$ and call this a base of $h_{K}$. In particular, when $n>1, B_{h}=D_{n} \cup_{\partial} D^{2}$, so denote $B_{h}$ 
by $\widehat{D}_{n}$. (This is an abuse of notation; $\widehat{D}_{n}$ is not a closed surface.) Then let

$$
h_{K}=\left\{\begin{array}{r}
\left\{\text { the components of } N(C), \text { the components of } \overline{F^{\prime}-N(C)}, B_{h}\right\} \\
\text { if } \left.B_{h} \text { is not an annulus (e.g., } n>2\right), \\
\text { the components of } N\left(C-\partial B_{h}\right), \text { the components of } \\
\text { if } \left.B_{h} \text { is an annulus (e.g., } n=2\right),
\end{array}\right.
$$

where $F^{\prime}=\overline{F-B_{h}}$ and $N(\cdots)$ denotes a regular neighborhood in $F^{\prime}$ (Figure 3).

First isotop $\hat{f}$ rel $C$ so that $\hat{f}\left(h_{K}\right)=h_{K}$. By Casson and Gordon [4, Theorem 5.1] $\hat{f}: \widehat{F} \rightarrow \widehat{F}$ extends to an automorphism $\varphi$ of a handlebody $H_{K}$, say. Then $h_{K}$ is a complete boundary pattern of $H_{K}$ and $\varphi$ is an admissible automorphism of $\left(H_{K}, h_{K}\right)$ in the sense of Johannson [19, p. 19].

For a component $S \in h_{K}-\left\{B_{h}\right\}$ define the root component $\partial_{r} S$ of $\partial S$ to be the component of $\partial S$ separating $B_{h}$ from the other components of $\partial S$ in $\partial H_{K}$. For $S \in h_{K}$ let $m$ be the least positive integer such that $\hat{f}^{m}(S)=S$. We call $\hat{f}^{m} \mid S: S \rightarrow S$ the adjoint automorphism of $S$, and denote it by $\hat{f}_{S}$. These are natural extensions of the definitions of a root boundary component and an adjoint automorphism for the closure of a component of $F-C$ in $\S 3$. Let us deform $\hat{f}$ by using the lemmas in $\S 3$.

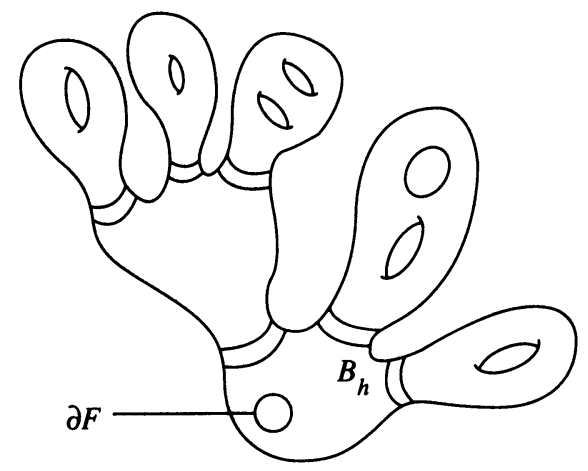

or

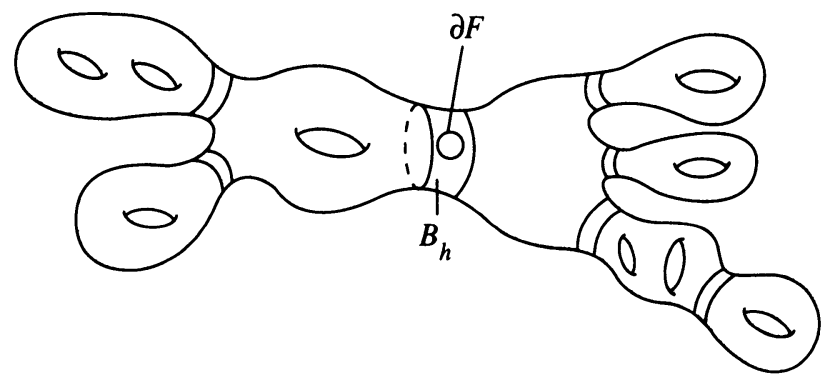

FIGURE 3. The boundary pattern of $h$ 
Proposition 5.1. After an isotopy $\hat{f}:\left(\widehat{F}, h_{K}\right) \rightarrow\left(\widehat{F}, h_{K}\right)$ satisfies the following. Let $M$ denote the ambient manifold of $K$.

1. $\hat{f}\left(h_{K}\right)=h_{K} ; \hat{f}_{A}=$ id for any annulus component $A \in h_{K}-\left\{B_{h}\right\}$.

2. $\hat{f}\left(B_{h}\right)=B_{h}, \hat{f} \mid \widehat{D}_{n}=\mathrm{id}$.

3. For any $S \in h_{K}-\left\{B_{h}\right\} \hat{f}_{S}: S \rightarrow S$ is irreducible or isotopic to a periodic map.

4. (a) Take $R,(\neq) S \in h_{K}-\left\{B_{h}\right\}$ adjacent to the same annulus component $A \in h_{K}-\left\{B_{h}\right\}$. If $f^{k}$ preserves $R \cup A \cup S$ for some $k>0$, then $f^{k} \mid R \cup A \cup S$ is not isotopic to a periodic map on $R \cup A \cup S$.

(b) If $n>2$, then (a) holds also when $R$ or $S=\widehat{D}_{n}$.

5. If $M$ is a homotopy sphere, then for any $S \in h_{K}-\left\{B_{h}\right\} \hat{f}_{S}$ is either the identity on a punctured disk, or irreducible and not isotopic to the identity.

6. If $M$ is a homotopy sphere, then for any $S \in h_{K}-\left\{B_{h}\right\} \hat{f}_{S}:\left(S, \partial_{r} S\right) \rightarrow$ $\left(S, \partial_{r} S\right)$ is the monodromy of a fibered knot in a homotopy sphere with unknotted holes $Y$ (say), so that $\partial_{r} S=\partial_{*} S$. Moreover, if $M=S^{3}$, then $Y$ is the exterior of a trivial link in $S^{3}$.

Proof. These are direct consequences of assertions 1-6 in Lemmas 3.1-3.3. We only remark on the exclusion of $B_{h}$. Let $B_{h}^{\prime}$ be the closure of the component of $F-C$ containing $\partial F$; then $B_{h}=B_{h}^{\prime} \cup D^{2}$. In general, even if $f \mid B_{h}^{\prime}$ is irreducible (resp. not isotopic to a periodic map), $\hat{f} \mid B_{h}$ is possibly reducible (resp. isotopic to a periodic map). But both $f \mid B_{h}^{\prime}$ and $\hat{f} \mid B_{h}$ are the identity when $n>1$ (cf. part 4(b)).

Here we can state our fundamental theorems.

Theorem 5.2. Let $K_{1}, \ldots, K_{n}(n \geq 2)$ be prime fibered knots in homology spheres such that $K=K_{1} \# \ldots \# K_{n} \geq 0$. Define $H_{K}, \widehat{D}_{n}$ as above.

1. Assume that $\partial H_{K}-\widehat{D}_{n}$ is incompressible in $H_{K}$. Then $\{1, \ldots, n\}$ can be paired into $\bigsqcup_{s=1}^{m}\left\{i_{s}, j_{s}\right\}$ such that

$$
K_{i_{s}} \# K_{j_{s}} \geq 0 \text { for all } s .
$$

2. There are (possibly composite or trivial) fibered knots $\widetilde{K}_{i}, 1 \leq i \leq n$, in homology spheres satisfying the following.

(a) $K_{i} \geq \widetilde{K}_{i}$ and $K^{\prime}=\#_{i=1}^{n} \widetilde{K}_{i} \geq 0$.

(b) $K^{\prime}$ satisfies the assumption in part 1 , i.e., $\partial H_{K^{\prime}}-\widehat{D}_{l}$ is incompressible in $H_{K^{\prime}}$ where $l$ is the number of factors in the prime decomposition of $K^{\prime}$.

For a composite fibered knot in a homotopy sphere, we can obtain finer results under a weaker assumption stated in terms of the boundary pattern $h_{K}$. Specifically, the assumption of Theorem 5.2 part 1 is stronger than needed in this case.

A component $S \in h_{K}$ is reducible if $\hat{f}_{S}: S \rightarrow S$ is reducible. If $S$ is not an annulus and $\hat{f}_{S}$ is isotopic to the identity, then $S$ is called a trivial component of $h$. For nonannulus components $S_{0}, S_{1}$ of $h_{K}-\left\{B_{h}\right\}$ take the fibered knots $J_{0}, J_{1}$ with monodromies $\hat{f}_{S_{i}}:\left(S_{i}, \partial_{r} S_{i}\right) \rightarrow\left(S_{i}, \partial_{r} S_{i}\right)$. We write $S_{1}=S_{0}^{*}$ if $J_{1} \cong J_{0}^{*}$. 
Theorem 5.3. Let $K_{1}, \ldots, K_{n}(n \geq 2)$ be prime fibered knots in homotopy spheres such that $K=K_{1} \# \cdots \# K_{n} \geq 0$. Define $F_{i}, 1 \leq i \leq n, H_{K}, h_{K}, \widehat{D}_{n}$ as above. Assume that each nontrivial component of $h_{K}$ is incompressible in $H_{K}$. Then the following hold.

1. For each $i, 1 \leq i \leq n$, there are prime fibered knots $K_{j}^{i}, 1 \leq j \leq$ $n(i)$, in homotopy spheres (or $S^{3}$ if $K_{i}$ is a knot in $S^{3}$ ) such that $K_{i} \geq \#_{j=1}^{n(i)} K_{j}^{i}$ and $\left\{K_{j}^{i}\right\}_{i, j}$ can be paired into $\coprod_{s=1}^{m}\left\{K_{j_{s}}^{i_{s}}, K_{j_{s}^{\prime}}^{i_{s}^{\prime}}\right\}$ such that $K_{j_{s}}^{i_{s}} \cong-K_{j_{s}^{\prime}}^{i_{s}^{\prime}}$.

2. Suppose that some $K_{i_{0}}$ does not have a satellite presentation $J(k \#-k)$ or $\left.J_{1}\left(J_{\left(J^{*}\right.}(k)\right)\right)$ where $k \neq 0$ and $J \not\left(S^{1} \times D^{2}, S^{1} \times 0\right)$. Then we may choose $n\left(i_{0}\right)=1$ and $K_{i_{0}}=K_{1}^{i_{0}}$ in part 1 . The second assumption can be replaced by the following: there are no $S_{0}, S_{1} \in h_{K} \cap F_{i_{0}}$ such that $S_{0}$ and $S_{1}$ are adjacent via an annulus, and $S_{1}=S_{0}^{*}$.

Remark. It is not difficult to see $J(k \#-k) \geq J(0)$. Proposition 4.1 shows $J_{1}\left(J\left(J^{*}(k)\right)\right) \geq J_{1}(k)$. (In each inequality, if the first knot is in $S^{3}$, so is the second one and " $\geq$ " is ribbon concordance [11].) These facts justify the assumptions in part 2. In fact, $\#_{j} K_{j}^{i}$ in part 1 is obtained from $K_{i}$ by performing these two types of reductions repeatedly.

Theorem 5.4. Let $K=J(k)$ be a fibered knot in a homotopy sphere such that $K \geq 0, k \neq 0$, and $J$ is a cable of $S^{1} \times D^{2}$. If each nontrivial component of $h_{K}$ is incompressible in $H_{K}$, then $J \cong\left(S^{1} \times D^{2}, S^{1} \times 0\right)$, a trivial cable.

Remark. Theorem 5.4 does not necessarily hold for a cable knot in a homology sphere. Let $N$ be the homology sphere obtained by a $-\frac{1}{2}$-Dehn surgery of $S^{3}$ on the figure eight knot. Let $k$ denote the core of the glued solid torus, and $K(\subset N)$ the $(2,1)$ cable of $k$. Then the closed monodromy of $K$ extends over a handlebody with the assumption of Theorem 5.4 satisfied. For the proof see Example 3 in $\S 8.1$.

Proof of Theorem 5.2, part 2. Take a maximal compression body $B$ of $\overline{\partial H_{K}-\widehat{D}_{n}}$ in $H_{K}$. Now $\overline{\partial H_{K}-\widehat{D}_{n}}$ is the disjoint union of the fibers of $K_{i}, \quad 1 \leq i \leq n$, so $B$ consists of $n$ compression bodies $B_{1}, \ldots, B_{n}$ such that $\partial_{+} B_{i}=F_{i}$. Since $B$ is maximal, $\partial_{-} B_{i}$ is incompressible in $H_{K}$, hence $\partial_{-} B_{i}$ has no closed surface components. This implies that $\partial_{-} B_{i}$ is connected for each $i$. Recall that we denote by $\varphi$ an extension of the closed monodromy of $K$ over $H_{K}$. The maximality of $B$ implies that $\varphi(B)=B$ after an isotopy, so that $\varphi\left(B_{i}\right)=B_{i}$. Let $\widetilde{K}_{i}$ be the fibered knot in a homology sphere with monodromy $\varphi \mid \partial_{-} B_{i}$. By Lemma $1.2 \varphi \mid B_{i}: B_{i} \rightarrow B_{i}$ induces a homotopically ribbon concordance $K_{i} \geq \widetilde{K}_{i}$. Let $H^{\prime}=\overline{H_{K}-B}$. Then $\varphi \mid H^{\prime}$ is an automorphism of the handlebody $H^{\prime}$ and $\varphi \mid \partial H^{\prime}$ is the closed monodromy of the fibered knot $\#_{i=1}^{n} \widetilde{K}_{i}$, therefore $\#^{n} \widetilde{K}_{i} \geq 0$ (assertion (a)). We have already shown that $\overline{\partial H^{\prime}-\widehat{D}_{n}}=\amalg_{i} \partial_{-} B_{i}$ is incompressible in $H^{\prime}$, implying assertion (b).

Our main task is to prove Theorem 5.2, part 1, and Theorems 5.3 and 5.4. We first see that several results in the Introduction are immediate consequences of these theorems. 
Theorem 5.5. Let $K_{1}, \ldots, K_{n}$ be prime fibered knots in homology (resp. homotopy) spheres $M_{i}$ such that $K=K_{1} \# \cdots \# K_{n} \geq 0$. If each $K_{i}$ satisfies either condition 1 or 2 below, then $\{1, \ldots, n\}$ can be paired into $\amalg_{s=1}^{m}\left\{i_{s}, j_{s}\right\}$ such that $K_{i_{s}} \# K_{j_{s}} \geq 0$ (resp. $K_{i_{s}} \cong-K_{j_{s}}$ ).

1. $K_{i}$ is minimal with respect to " $\leq$ " among all fibered knots in homology spheres.

2. There is no $f(t) \in Z[t]-\left\{t^{k}\right\}_{k}$ such that $f(t) f\left(t^{-1}\right) \mid \Delta_{K_{i}}(t)$.

Proof. By Lemma 1.2 each of conditions 1 and 2 implies that the monodromy of $K_{i}$ does not extend over a nontrivial compression body $B$ with $\partial_{-} B$ connected. From the proof of Theorem 5.2 part $2 \overline{\partial H_{K}-\widehat{D}_{n}}$ has a trivial maximal compression body, hence it is incompressible in $H_{K}$. Applying Theorem 5.2 part 1 to $K$ proves Theorem 5.5 for knots in homology spheres. Now suppose all $M_{i}$ are homotopy spheres. If each $K_{i}$ is minimal, by the remark just after Theorem 5.3 $K_{i}$ cannot have the satellite presentations precluded in the hypothesis of Theorem 5.3 part 2 for any $i$. Then, applying Theorem 5.3 part 2 to $K$ gives the desired pairings. To complete the proof let us show condition 2 implies condition 1 . Suppose $K_{i}$ does not satisfy condition 1 . Then $K_{i}$ is homotopically ribbon concordant to some fibered knot $L$ with $\operatorname{deg} \Delta_{K_{i}}>\operatorname{deg} \Delta_{L}$ (cf. the proof of Lemma 1.1). Hence by [1, p. 541] $\Delta_{K_{i}}(t)$ is divisible by a nontrivial Laurent polynomial $f(t) f\left(t^{-1}\right)$, violating condition 1 .

Theorem 5.6. Let $K$ be a (possibly composite) nontrivial fibered knot in a homology (resp. homotopy) sphere such that $n K \geq 0$ for some $n>0$. If $K$ satisfies either condition 1 or 2 in Theorem 5.5, then $2 K \geq 0$ (resp. $K$ is -amphicheiral).

Proof. Without loss of generality assume that $n$ is even. (If necessary, take $2 n$.) Let $K=k_{1} \# \cdots \# k_{l}$ be a prime decomposition of $K$. Then each $k_{i}$ satisfies condition 1 or 2 in Theorem 5.5. Before applying Theorem 5.5 to $n K$, identify a set of $l$ vertices $V=\{1, \ldots, l\}$ with $\left\{k_{1}, \ldots, k_{l}\right\}$. The pairings given by Theorem 5.5 yield a set of unoriented edges $E$ for $V$ by connecting $i$ and $j \in V$ such that $k_{i} \# k_{j} \geq 0$; the degree of each vertex is $n$. In other words, $(E, V)$ is an $n$-regular graph. By Petersen's theorem $[16$, p. 36] even regular graphs are 2 -factorable. This means that the $2 l$ knots $k_{1}, k_{1}, k_{2}, k_{2}, \ldots, k_{l}, k_{l}$ can be paired into $\bigsqcup_{s=1}^{l}\left\{k_{i_{s}}, k_{j_{s}}\right\}$ such that $k_{i_{s}} \# k_{j_{s}} \geq 0$. Therefore $2 K \geq 0$. Now assume that $K$ is a knot in a homotopy sphere; then $k_{i_{s}} \cong-k_{j_{s}}$. If $i_{s} \neq j_{s}$ for some $s$, then $K$ is not minimal, contradicting conditions 1 and 2 . It follows $k_{i} \cong-k_{i}$ for $1 \leq i \leq l$, hence $K \cong-K$.

The following question, an analog of the ribbon-slice problem, is a slight modification of Question 4.22(B) in [21].

Question 5.7. Let $K$ be a knot in a homology sphere $M$. Suppose that $M$ bounds a contractible 4-manifold $V$ and $K$ bounds a disk in $V$. Then, is $K$ homotopically ribbon?

An affirmative answer to this question improves Theorem 5.6 dramatically.

Theorem 5.8. Let $K$ be a fibered knot in a homology sphere such that $n K \geq 0$ for some $n>0$. If Question 5.7 has an affirmative answer, then $2 K \geq 0$. 
Corollary 5.9. If Question 5.7 has an affirmative answer and the 4-dimensional Poincaré conjecture holds, then the order of a classical knot concordance class represented by a fibered knot is 1,2 , or $\infty$.

Proof of Theorem 5.8. Let $M$ be the ambient manifold of $K$. By Lemma 1.1 there is a fibered knot $K^{\prime}$ in a homology sphere $M^{\prime}$ which is minimal among all fibered knots $\leq K$. Let $V$ be a homology 4-ball such that $\partial V=n M$ and $n K$ bounds a homotopically ribbon disk in $V$. Let $W$ be a 4-manifold such that $\partial W=n M \amalg n\left(-M^{\prime}\right), n K \geq n K^{\prime}$ in $W$. Set $X^{4}=-V \cup_{n M} W$. Since $(1=)$ $\pi_{1}(M) \rightarrow \pi_{1}(V)$ and $\pi_{1}(M) \rightarrow \pi_{1}(W)$ are surjective, $X^{4}$ is contractible. The fibered knot $n K^{\prime}$ bounds a smooth disk in $-X^{4}$. The theorem's assumption implies $n K^{\prime} \geq 0$. Therefore, Theorem 5.8 follows from Theorem 5.6.

Our first step toward Theorems 5.2-5.4 is to prove the following lemma.

Lemma 5.10. The proofs of Theorems 5.2, part 1, 5.3, and 5.4 reduce to the case when every component of $h_{K}$ is incompressible in $H_{K}$.

Proof. We first show the assertion about Theorem 5.3. Suppose that Theorem 5.3 holds for $K$ if every component of $h_{K}$ is incompressible in $H_{K}$. Let $\alpha(K)=\left|h_{K}\right|$. We then prove Theorem 5.3 by induction on $\alpha(K)$. Since $n \geq 2$, $\alpha(K) \geq 3$. When $\alpha(K)=3, h_{K}$ contains only nontrivial components, hence Theorem 5.3 holds in this case.

Take a fibered knot $K=K_{1} \# \cdots \# K_{n}$ satisfying the assumption of Theorem 5.3. Assume that Theorem 5.3 holds for all $K^{\prime}$ such that $\alpha\left(K^{\prime}\right)<\alpha(K)$. Without loss of generality there is a trivial component, $S$, of $h_{K}$ compressible in $H_{K}$. By Proposition 5.1 parts 4 and 5 trivial components cannot be adjacent to each other via annuli. Combining this with the theorem's assumption, we see that a boundary of a compression disk $D$ for $S$ is not parallel to $\partial S$ in $\partial H$.

Case 1. $S=\widehat{D}_{n}$. The above observation implies $n>2$. Without loss of generality, on $\partial H_{K}$ the circle $\partial D$ separates $\overline{\partial H_{K}-\widehat{D}_{n}}=\coprod_{i=1}^{n} F_{i}$ into $F_{1}, \ldots, F_{l}$ and $F_{l+1}, \ldots, F_{n}$, where $1<l<n-1$. Isotop $\varphi$ rel $\partial H_{K}$ so as to fix $D$. Then the closure of components of $H_{K}-D$ consists of two handlebodies $H_{1}$ and $H_{2}$. The induced automorphisms, $\varphi_{i}$, of $\partial H_{i}$ are the closed monodromies of $\#^{l} K_{i}=L_{1}$ and $\#_{l+1}^{n} K_{i}=L_{2}$; note that $h_{L_{i}}-\left\{B_{L_{i}}\right\} \subset h_{K}-\left\{\widehat{D}_{n}\right\}$. Then for $i=1,2$ we have $L_{i} \geq 0, \alpha\left(L_{i}\right)<\alpha(K)$ and every nontrivial component of $h_{L_{i}}$ is incompressible in $H_{L_{i}}$. By the induction hypothesis Theorem 5.3 holds for $L_{1}$ and $L_{2}$, and hence for $K$.

Case 2. $S \neq \widehat{D}_{n}$. Let $s=|\partial S|-1(>1)$. Without loss of generality $S \subset F_{1}$. Then $K_{1}$ has a satellite presentation $J_{0}\left(k_{1} \# \ldots \# k_{s}\right)$ where $k_{i}$ is a prime knot. Note that $J_{0}$ is prime, for $K_{1}$ is prime. Since $\left\{\partial \varphi^{i}(D)\right\}_{i}$ is a set of disjoint simple loops in $\partial H_{K}$, after an isotopy $\left\{\varphi^{i}(D)\right\}_{i}$ consists of disjoint disks in $H_{K}$. Cutting $H_{K}$ along $\bigcup_{i} \varphi^{i}(D)$, we obtain handlebodies $H_{0}, H_{1}, \ldots, H_{m}$ such that $\varphi\left(H_{0}\right)=H_{0}$; then $\varphi^{m}\left(H_{1}\right)=H_{1}$. Without loss of generality we see that $\varphi \mid \partial H_{0}$ (resp. $\left.\varphi^{m} \mid \partial H_{1}\right)$ is the monodromy of $J_{0}\left(k_{1} \# \cdots \# k_{l}\right) \# K_{2} \# \cdots \# K_{n}=$ $K^{\prime}$, say, (resp. $k_{l+1} \# \cdots \# k_{s}=L$, say), where $1<l<s$. Hence, by Casson and Gordon [4, Theorem 5.1] $K^{\prime} \geq 0$ and $L \geq 0$. Since the boundary patterns $h_{K^{\prime}}, h_{L}$ are induced from $h_{K}$, it follows that $\alpha\left(K^{\prime}\right)<\alpha(K), \alpha(L)<\alpha(K)$, and both $h_{K^{\prime}}$ and $h_{L}$ satisfy the assumption of Theorem 5.3.

A regular neighborhood, $B$, of $F_{1} \cup\left(\bigcup_{i=1}^{m} H_{i}\right)$ in $H_{K}$ is a compression body such that $\varphi(B)=B, \partial_{+} B=F_{1}$, and $\partial_{-} B$ is the fiber of a prime knot 
$J_{0}\left(k_{1} \# \cdots \# k_{l}\right)=K_{1}^{\prime}$, say. It follows from Lemma 1.2 that $K_{1} \geq K_{1}^{\prime}$. Applying Theorem 5.3 to $K^{\prime}$ shows that Theorem 5.3, part 1 holds for $K$. Next suppose that $K$ satisfies the assumption of Theorem 5.3, part 2; then so does $L=k_{l+1} \# \cdots \# k_{s}$. By applying Theorem 5.3 , part 2 to $L$, we obtain $-k_{s} \cong k_{j}$ for some $l+1 \leq j \leq s$. Therefore $K_{1}$ has a presentation $J\left(k_{s} \#-k_{s}\right)$ for some $J$, a contradiction. Theorem 5.3, part 2 holds for $K$.

The assertion about Theorem 5.2, part 1 and Theorem 5.4 can be proved by the similar arguments. To prove the reduction of Theorem 5.2, part 1 (resp. Theorem 5.4) it suffices to consider only Case 1 (resp. Case 2) above. This completes the proof of Lemma 5.10.

Therefore, to prove Theorems 5.2-5.4 we may assume that every component of $h_{K}$ is incompressible in $H_{K}$. Let us see that $h_{K}$ is a useful, complete boundary pattern in the sense of [19]. Suppose not; then there is an $i$-faced disk $D$, $1 \leq i \leq 3$, in $\left(H_{K}, h_{K}\right)$. Since each boundary component of a surface in $h_{K}$ is separating in $\partial H_{K}, D$ is 1 -faced or 2 -faced. Recalling that every nonannulus component of $h_{K}$ is adjacent to an annulus in $h_{K}$, isotop $D$ so that $\partial D$ is contained in a component of $h_{K}$. Then such a component is compressible in $H_{K}$, a contradiction.

Since $h_{K}$ is useful, by $[19,9.4]\left(H_{K}, h_{K}\right)$ has a characteristic submanifold $X_{K}$, which consists of essential $I$-bundles and essential Seifert fibered manifolds. Define $U_{K}$ to be the union of $I$-bundle components of $X_{K}$ not homeomorphic to $S^{1} \times D^{2}$, and let $V_{K}=X_{K}-U_{K}$. We shall see that $V_{K}$ consists of essential Seifert fibered manifolds. Since $\varphi$ is an admissible automorphism of $\left(H_{K}, h_{K}\right), \varphi\left(X_{K}\right)$ is also a characteristic submanifold for $\left(H_{K}, h_{K}\right)$. By [19, 10.9] we can isotop $\varphi$ admissibly with respect to $h_{K}$ so that $\varphi\left(X_{K}\right)=X_{K}$. After this isotopy, we have $\varphi\left(U_{K}\right)=U_{K}, \varphi\left(V_{K}\right)=V_{K}$. For simplicity let $H, h, X, U, V$ denote $H_{K}, h_{K}, X_{K}, U_{K}, V_{K}$ respectively.

In the next two subsections we shall study the position of $U$ and $V$ in the handlebody $H$. We shall use the terminology of Johannson [19]. Refer to [19] for the definitions of essential surfaces, admissible I-bundles, admissibly parallel, complete manifolds, etc.

5.2. Position of $U$ and $V(1)$. Let $K$ be a fibered knot in a homology sphere such that $K \geq 0$. Define $n, f, \varphi, B_{h}, \widehat{D}_{n}, H, h, X, U, V$ as in $\S 5.1$; in particular, we assume that $h$ is a useful boundary pattern. At the end of this subsection the proofs of Theorems 5.2-5.4 reduce to the case when $U$ contains no annulus components of $h-\left\{B_{h}\right\}$.

Lemma 5.11. 1. Each component of $\operatorname{Fr} X$ is an essential annulus in $(H, h)$ such that each boundary component separates $\partial H$. Furthermore, each component of Fr $X$ separates $H$.

2. $V$ consists of essential Seifert fibered manifolds in $(H, h)$.

Proof. Let $M$ be a component of $X$. If $M$ is an essential Seifert fibered manifold, then $\operatorname{Fr} M$ consists of essential annuli or essential tori by the definition. Since a handlebody contains no essential tori, each component of $\operatorname{Fr} M$ is an essential annulus. Suppose that $M$ is an $I$-bundle. If $\operatorname{Fr} M$ contains a component other than an annulus, then for some $S_{1}$ in $h \partial M \cap S_{1}$ contains a square component connecting the lids of $M$. Let $S_{2}$ be a component of $h$ containing one of the lids of $M$; then $S_{2}$ cannot be an annulus. Since $S_{2}$ is adjacent to 
$S_{1}, S_{1}$ is an annulus. By part 2 of 10.6 of [19] the characteristic submanifold $X$ contains the annulus component of $h$ entirely, hence $S_{1}$ is not an annulus, a contradiction. Thus $\operatorname{Fr} X$ consists of annuli.

Let $N$ denote the ambient manifold of $K$. Let $c$ be a component of $\partial(\operatorname{Fr} X)$, an essential simple loop in $\partial H$. Since $\varphi(X)=X$, for some positive integer $k$ we have $\varphi^{k}(c)=c$. Thus $c$ is a component of the intersection of $F$ and the torus $\left(\bigcup_{i=1}^{k} \varphi^{i}(c) \times I\right) / \varphi$ in $N-N(K)$. Since this torus separates $N$, the union of simple loops $\bigcup_{i=1}^{k} \varphi^{i}(c)$ separates $F$. If $k=1$, the simple loop $c$ separates $F$. If $k>1$ and $c$ is nonseparating, then homology calculation shows $H_{1}(N)$ has a nontrivial torsion element, contradicting the fact that $N$ is a homology sphere. Thus $c$ separates $F$.

Let $A$ be a component of $\operatorname{Fr} X$. Then $\partial H-\partial A$ has three components. Let $G$ be the union of two components of $\partial H-\partial A$ each of which has only one boundary component. If $A$ did not separate $H$, then there would be a loop in $H$ meeting the closed surface $G \cup A$ in a single point. Since $H \subset S^{3}$, this is a contradiction. This completes the proof of part 1.

Let $M$ be an $I$-bundle component of $X$ homeomorphic to $S^{1} \times D^{2}$. By part $1 M \cap \partial H$ consists of tori or annuli. Thus we may regard $M$ as an essential Seifert fibered manifold in $(H, h)$, completing the proof of part 2 .

Lemma 5.12. If $T$ is a component of the lids of $U$, then $T$ is contained in a nonannulus component, $S$, of $h$ such that $T$ is isotopic to $S$ in $\partial H$, or $T$ is contained in a reducible component of $h$.

Proof. By the definitions of $U$ and an admissible $I$-bundle each component of the lids of $U$ is contained in a nonannulus component of $h$. Suppose that $S$ is an irreducible component of $h$, and $T$ is one of the lids of $U$ contained in $S$. Then $T$ is a component of $X \cap S$. Note that $\bigcup_{i}\left(\hat{f}_{S}\right)^{i}(\partial T)$ consists of pairwise disjoint, essential simple loops in $S$ and is kept invariant under the adjoint automorphism $\hat{f}_{S}$. Hence $\bigcup_{i}\left(\hat{f}_{S}\right)^{i}(\partial T)$ is isotopic to $\partial S$, so that $T$ is isotopic to $S$ in $\partial H$.

Lemma 5.13. All components of $U$ are admissible product I-bundles.

Proof. Suppose that a component $U_{0}$ of $U$ is a twisted $I$-bundle. Let $T$ be the lid of $U_{0}$. Then $\overline{\left(U_{0} \cap \partial H\right)-T}$ is a (possibly empty) set of annuli each of which connects different components of $\partial T$. If there is an annulus, then some component of $\partial T$ is nonseparating in $\partial H$, contradicting Lemma 5.11. It follows that $U_{0} \cap \partial H=T$, which is connected. Since each component of $\partial T$ separates $\partial H$, each component of $\operatorname{Fr} U_{0}$ is separating in $\partial\left(\overline{H-U_{0}}\right)$. Hence $U_{0}(\subset H)$ satisfies the hypothesis of the lemma below.

Lemma 5.14. Let $M$ be a 3-manifold in a handlebody $H$ such that Fr $M$ consists of separating incompressible annuli each of which does not separate $\partial M$ but $\partial(\overline{H-M})$. Let $\alpha_{1}, \ldots, \alpha_{m}$ be the cores of components of $\operatorname{Fr} M$. Then $M$ is a handlebody such that $\left\{\alpha_{1}, \ldots, \alpha_{m}\right\}$ is primitive, i.e., there are disjoint disks $D_{1}, \ldots, D_{m}$ in $M$ such that $\left|\alpha_{i} \cap D_{j}\right|=\delta_{i j}$.

The proof of Lemma 5.14 is deferred until after the proof of Lemma 5.13 we are in. Identify $U_{0}$ with a twisted $I$-bundle $S \times{ }_{\tau} I$ where $S$ is a connected nonorientable surface. It follows from Lemma 5.14 that the set of cores of 
Fr $U_{0}$ is primitive for the handlebody $U_{0}$. Then each component of $\partial S \times_{\tau}\left\{\frac{1}{2}\right\}$ represents a generator of $H_{1}\left(S \times_{\tau} I\right)$, absurd. Lemma 5.13 is proved.

Lemma 5.14 is one of the key lemmas in this paper. It is unnecessarily strong for proving Lemma 5.13, but we shall need it essentially in $\S 5.3$.

Proof of Lemma 5.14. Let $A_{1}, \ldots, A_{m}$ be the components of Fr $M$ such that the core of $A_{i}$ is $\alpha_{i}$. Identifying $A_{i}$ with $\left(S^{1} \times I\right)_{i}$ (a copy of $\left.S^{1} \times I\right)$, let $B_{i}=(\gamma \times I)_{i}$ where $\gamma$ is a half circle of $S^{1}$. Let $M_{1}, \ldots, M_{m}$ be the components of $\overline{H-M}$. Denote the copy of $A_{i}$ in $\overline{H-M}$ by $A_{i}^{\prime}$; then $A_{i}^{\prime}$ separates $\partial M_{i}$. Also denote the copy of $B_{i}$ in $M_{i}$ by $B_{i}^{\prime}(1 \leq i \leq m)$. Let $H^{\prime}$ be the 3-manifold obtained from $M$ and $M_{i}, 1 \leq i \leq m$, by pasting $B_{i}$ to $B_{i}^{\prime}$ for all $i$ naturally. Then $H \cong H^{\prime} \cup \bigcup_{i=1}^{m} h_{i}$ where $h_{i}$ is a 2-handle with $h_{i} \cap H^{\prime}=\overline{A_{i}-B_{i}} \cup \overline{A_{i}^{\prime}-B_{i}^{\prime}}$. Note that for any subset $C$ of $\{1, \ldots, m\}$ the manifold $H^{\prime} \cup \bigcup_{i \in C} h_{i}$ is homotopy equivalent to the disk sum of $H^{\prime \prime}=$ $\overline{H-\bigcup_{i \notin C} M_{i}}$ and all $M_{i}, i \notin C$. Since $A_{i}$ is incompressible in $H$, the groups $\pi_{1}\left(H^{\prime \prime}\right), \pi_{1}\left(M_{i}\right)$ and $\pi_{1}(M)$ are subgroups of the free group $\pi_{1}(H)$. Hence $H^{\prime} \cup\left(\bigcup_{i \in C} h_{i}\right)$ is a handlebody for any $C$, and so is $M$.

Let $c_{1}, \ldots, c_{m}\left(\subset \partial H^{\prime}\right)$ denote the attaching circles of the 2-handles $h_{i}$ such that $\left|c_{i} \cap B_{j}\right|=2 \delta_{i j}$. Then, by [14, Theorem 1] we see that $\left\{c_{1}, \ldots, c_{m}\right\}$ is primitive for the handlebody $H^{\prime}$. Take disjoint disks $D_{1}, \ldots, D_{m}$ in $H^{\prime}$ such that $\left|c_{i} \cap \partial D_{j}\right|=\delta_{i j}, c_{i} \cap \partial D_{j} \subset M$, and $\left|\left(\bigcup B_{i}\right) \cap\left(\bigcup D_{j}\right)\right|$ is minimal among all sets of such disks. Let us show $\cup D_{j} \subset M$, which completes the proof. Suppose not; then there is an arc $\beta$, a component of $B_{i} \cap D_{j}$ for some $i, j$. Assume that $\beta$ bounds a disk $\Delta$ in $B_{i}$ with $\Delta \cap c_{i}=\varnothing$. Compressing $\bigcup D_{j}$ in $H^{\prime}$ via $\Delta$ yields a set of dual disks for $\left\{c_{i}\right\}$ which has fewer intersection with $\bigcup B_{i}$ than $\bigcup D_{j}$ has, a contradiction. Hence, after an isotopy of $\bigcup D_{j}, B_{i} \cap D_{j}$ consists of arcs $p \times I\left(\subset B_{i}\right)$ where $p \in \gamma$ (cf. Figure 4).

Without loss of generality let $\left(\bigcup B_{i}\right) \cap D_{1} \neq \varnothing$. Consider the components of

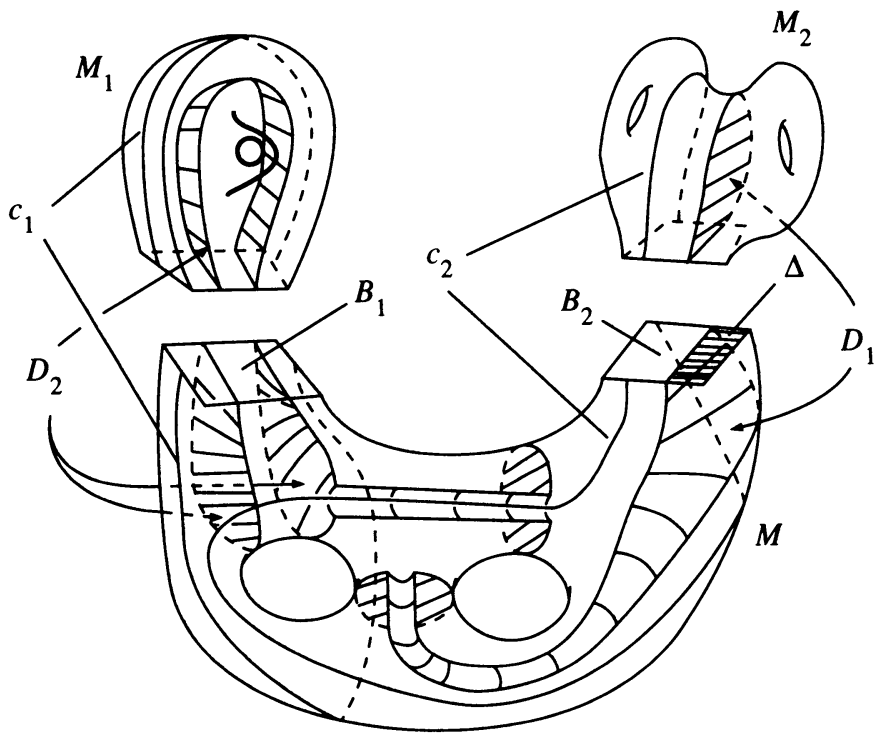

FIGURE 4 
$D_{1}-\bigcup B_{i}$ in $D_{1}$ and let $E$ be the closure of an outermost one in $D_{1}$ such that

$$
c_{i} \cap \partial E=\varnothing \text { for all } i \text {. }
$$

The existence of $E$ is guaranteed by $\left|\left(\bigcup c_{i}\right) \cap \partial D_{1}\right|=1$. The arc $\partial E-\partial D_{1}$ is contained in some $B_{i_{0}}$; it follows that $\left|\alpha_{j} \cap \partial E\right|=\delta_{i_{0} j}$ for all $j$. Note that $E \subset M$ or $E \subset M_{i_{0}}$. However the latter implies that the components of $\partial A_{i_{0}}^{\prime}$ are connected by the arc $\partial E \cap \partial D_{1}$ in $\partial M_{i_{0}}$, a contradiction. Hence, $E \subset M$. Let $E^{\prime}$ be the closure of the component of $D_{1}-\bigcup B_{i}$ adjacent to $E$. Then $E^{\prime} \subset M_{i_{0}}$ and $\partial E^{\prime}-\partial D_{1} \subset B_{i_{0}}$. Since $c_{1} \cap D_{1} \subset M, \partial E^{\prime}-\partial D_{1}$ consists of more than one arcs in $B_{i_{0}}$. Therefore some component of $\partial E^{\prime}-\partial D_{1}$ does not bound an outermost disk of $D_{1}-\bigcup B_{i}$ with the property $(*)$ above. In other words we have shown the following.

Assertion. Let $\mathscr{C}$ be the set of arcs $B_{i_{0}} \cap\left(\bigcup D_{j}\right)$. Let $\mathscr{C}^{\prime}$ be the subset of $\mathscr{C}$ such that each $\beta \in \mathscr{C}^{\prime}$ bounds an outermost disk of $D_{j}-\bigcup B_{i}$ in some $D_{j}$ which does not meet any $c_{i}$. Then $\mathscr{C}^{\prime} \neq \varnothing$ and $\mathscr{C}-\mathscr{C}^{\prime} \neq \varnothing$.

We shall deduce a contradiction from Assertion. Under an identification $B_{i_{0}} \cong I \times I$ each arc in $\mathscr{C}$ is $x \times I$ for some $x$ and $\alpha_{i_{0}} \cap B_{i_{0}}=I \times \frac{1}{2}$. It follows from Assertion that there are $\beta_{i}=x_{i} \times I \in \mathscr{C}, i=1,2$, such that $\beta_{1} \in \mathscr{C}^{\prime}, \beta_{2} \in \mathscr{C}-\mathscr{C}^{\prime}$, and no elements of $\mathscr{C}$ are in $\left(x_{1}, x_{2}\right) \times I$ (without loss of generality $\left.x_{1}<x_{2}\right)$. Set $D=\left(x_{1}, x_{2}\right) \times I$. Suppose that $D_{j_{k}}$ contains $\beta_{k}$ for $k=1,2$. Let $E_{1}$ be the closure of the outermost disk of $D_{j_{1}}-\bigcup B_{i}$ bounded by $\beta_{1}$ such that $\left(\bigcup c_{i}\right) \cap E_{1}=\varnothing$. Let $E_{2}$ be the closure of a component of $D_{j_{k}}-\beta_{2}$ meeting $c_{j_{2}}$. Let $D_{j_{2}}^{\prime}=E_{1} \cup D \cup E_{2}$ and push it off $D_{j_{1}}$ and $D$. Then $\left|\left(\bigcup B_{i}\right) \cap D_{j_{2}}^{\prime}\right|<\left|\left(\bigcup B_{i}\right) \cap D_{j_{2}}\right|$. Define $D_{i}^{\prime}=D_{i}$ if $i \neq j_{2}$. The disjoint disks $D_{i}^{\prime}, 1 \leq i \leq m$, satisfy $c_{i} \cap \partial D_{j}^{\prime}=c_{i} \cap \partial D_{j}$ for all $i, j$ and have fewer intersections with $\bigcup B_{i}$ than $\bigcup D_{j}$ has. This is a contradiction, completing the proof of Lemma 5.14.

Lemma 5.15. The Seifert fibered manifold $V$ in $(H, h)$ satisfies the following.

1. $V$ consists of solid tori with $V \cap \partial H$ longitudinal annuli in $V$.

2. No pair of components of $V \cap \partial H$ are isotopic to each other in $\partial H$.

3. Let $T$ be a component of $V \cap \partial H$. Then one of the following holds.

(a) $T$ is a regular neighborhood of an annulus component of $h$.

(b) $T$ is contained in a reducible component, $S$, of $h$ such that the core of $T$ is not parallel to $\partial S$ in $\partial H$.

Proof. Let $V_{0}$ be a component of $V$. Each component of $\operatorname{Fr} V_{0}$ is nonseparating in $\partial V_{0}$. Since each component of $\partial\left(\operatorname{Fr} V_{0}\right)$ separates $\partial H$, there is a component $N$ of $\overline{H-V_{0}}$ such that the annulus $A_{0}=N \cap V_{0}$ separates $\partial N$. Note that $V_{0} \cup N$ is a handlebody, and $V_{0}$ satisfies the hypothesis on $M$ in Lemma 5.11. It follows that $V_{0}$ is a solid torus with $A_{0}$ longitudinal in $V_{0}$, completing the proof of part 1.

Assume that there are two components $T_{1}, T_{2}$ of $V \cap \partial H$ whose cores are parallel in $\partial H$. Let $A$ be an annulus component of $\overline{\partial H-T_{1} \cup T_{2}}$. If $A \cap U \neq \varnothing, A$ contains at least one of the lids of $U$. Then some component of $U$ is an $I$-bundle over an annulus, a contradiction to the definition of $U$. Hence $A \cap U=\varnothing$. With an adequate boundary pattern, $V^{\prime}=V \cup N(A)$ (where $N(\cdots)$ is a regular neighborhood in $H$ ) can be an essential Seifert fibered manifold in 
$(H, h)$ which is disjoint from $U$. Since $X=U \cup V$ is full [19, p. 85], so is $U \cup V^{\prime}$. However the complexity [19, p. 84] of $U \cup V^{\prime}$ is greater than that of $X$, contradicting $X$ being a characteristic submanifold. This completes the proof of part 2.

Since $\varphi(V)=V, \partial(V \cap \partial H)$ is an invariant 1-submanifold of $\partial H$ under $\varphi$. If $S(\in h)$ is not a reducible component, then the adjoint automorphism of $S$ is irreducible. Hence $\partial(V \cap \partial H) \cap S$ consists of simple loops in $S$ parallel to $\partial S$. Part 3 of Lemma 5.15 follows from the observation above and claim below.

Claim. Let $A$ be an annulus component of $h$, and $T$ be a component of $V \cap \partial H$ isotopic to $A$ in $\partial H$. Then $T$ is a regular neighborhood of $A$.

Proof. Assume that $T \not \supset A$. By part 2 of Proposition 10.6 of [19] $X \supset A$, so that $A \cap T=\varnothing$. As in the proof of part 2 we have just proved, if $A \cap U \neq \varnothing$, then $U$ has a component homeomorphic to $S^{1} \times D^{2}$, a contradiction. It follows that $A \subset V$. Then $T$ is isotopic to the component of $V \cap \partial H$ which contains $A$, contradicting part 2 .

The following lemmas show that the position of $U$ is closely related to $V$.

Lemma 5.16. Let $\alpha, \beta$ be components of $\operatorname{Fr} U, \operatorname{Fr} V$ respectively. If there is an annulus $A$ in $\partial H$ such that $\partial A$ consists of a component of $\partial \alpha$ and $a$ component of $\partial \beta$, and $A \cap V \subset \partial A$, then $\alpha$ is admissibly parallel to $\beta$.

Proof. As in the proof of Lemma 5.15 we have $A \cap U=\varnothing$, hence $A \cap X=\partial A$. Pushing $A$ into Int $H$ with $A \cap X=\partial A$, we obtain an essential annulus in $\left(H^{\prime}, h^{\prime}\right)$, where $H^{\prime}=\overline{H-X}$ and $h^{\prime}$ is the boundary pattern of $H^{\prime}$ induced from $h$ (cf. $[19$, p. 90]). By the completeness of a characteristic submanifold $[19,10.10] \alpha$ and $\beta$ cobound (an annulus) $\times I$.

Lemma 5.17. Assume that $U$ does not contain any annulus components of $h-$ $\left\{B_{h}\right\}$.

1. $V \cap \partial H$ contains all annulus components of $h-\left\{B_{h}\right\}$.

2. Let $\alpha$ be a component of $\operatorname{Fr} U$ such that a component of $\partial \alpha$ is contained in an irreducible component of $h$. Then $\alpha$ is admissibly parallel to Fr $V$.

Proof. Part 1 follows from the fact that every annulus component of $h$ is contained in $U$ or $V$. We obtain part 2 by using Lemma 5.16 with an aid of part 1 and Lemma 5.12 .

The following general lemma seems to be folklore. The proof given below exploits a uniqueness of Seifert fibration.

Lemma 5.18. Let $S$ be connected, orientable surface. Let $S_{1}\left(\neq D^{2}, S^{1} \times I\right)$ be a connected 2-submanifold of $S$ such that no component of $\partial S_{1}$ bounds a disk in $S$. Let $f$ be an automorphism of $S$ which preserves $S_{1}$ and is periodic up to isotopy. If there is an isotopy $g_{t}: S_{1} \rightarrow S_{1}$ of $f \mid S_{1}$ such that $g_{1}$ is periodic, then $g_{t}$ extends to an isotopy $f_{t}$ of $f$ such that $f_{1}$ is periodic.

Proof. Without loss of generality $S \neq S_{1}$; then the Euler number of $S$ is negative. Let $h_{t}$ be an isotopy of $f$ such that $h_{1}$ is periodic. Set $M=S \times I / f$ 
and $M_{1}=S_{1} \times I /\left(f \mid S_{1}\right)$. Give $M$ (resp. $\left.M_{1}\right)$ the Seifert fibration induced by the flow $\left(h_{t}(x), t\right)_{t}$ (resp. $\left.\left(g_{t}(x), t\right)_{t}\right)$. Deform the fibration of $M$ so that $\partial M_{1}$ consists of saturated tori. Then the Seifert fibration of $M$ is (after isotopy) an extension of that of $M_{1}$, for a Seifert fibration of $M_{1}$ is unique up to isotopy. We may assume each $S^{1}$-fiber of $M$ is transverse to each $S \times t$. Then the fibration of $M$ gives the claimed isotopy.

The lemmas below reduce the proofs of Theorems 5.3 and 5.4 to the case when $U$ does not contain any annulus components of $h_{K}-\left\{B_{h}\right\}$; then Lemma 5.17 is valid.

Lemma 5.19. Let $K_{1}, \ldots, K_{n}$ be prime fibered knots in homotopy spheres such that (1) $K=K_{1} \# \ldots \# K_{n} \geq 0$; (2) if $n=1, K_{1}$ is a $(p, q)$ cable of a nontrivial knot, where $p>1$. Assume that $h_{K}$ is useful, and $U_{K}$ contains an annulus component of $h_{K} \cap F_{i_{0}}\left(\subset h_{K}\right)$, where $F_{i_{0}}\left(\subset \partial H_{K}\right)$ is the fiber of some $K_{i_{0}}$. Without loss of generality assume $i_{0}=1$. Then the following hold.

1. There are $S_{0}, S_{1} \in h_{K} \cap F_{1}$ such that $S_{0}$ and $S_{1}$ are adjacent via an annulus and $S_{1}=S_{0}^{*}$. If Theorem 5.3 holds for fibered knots $L$ with $\alpha(L)<\alpha(K)$, then $K_{1}$ has a satellite presentation $J(k \#-k)$ or $J_{1}\left(J\left(J^{*}(k)\right)\right)$ where $J \not\left(S^{1} \times D^{2}, S^{1} \times 0\right)$ and $k \neq 0$.

2. There is a nontrivial (possibly composite) fibered knot $K_{1}^{\prime}$ such that $K^{\prime}=$ $K_{1}^{\prime} \# K_{2} \# \ldots \# K_{n} \geq 0$ and $K_{1} \geq K_{1}^{\prime}$. If $n=1, K_{1}^{\prime}$ is $a(p, q)$ cable of $a$ nontrivial knot.

3. With $K^{\prime}$ as above each nontrivial component of $h_{K^{\prime}}$ is incompressible in $H_{K^{\prime}}\left(\subset H_{K}\right)$.

4. With $K^{\prime}$ as above $\alpha(K)>\alpha\left(K^{\prime}\right)$, where $\alpha(K)=\left|h_{K}\right|$.

Lemma 5.20. The proofs of Theorems 5.2, part 1, 5.3, and 5.4 reduce to the case when $U_{K}$ contains no annulus components of $h_{K}-\left\{B_{h}\right\}$.

Proof of Lemma 5.19. Let $U_{0}$ be a component of $U$ containing an annulus component, $A$, of $h \cap F_{1}$. Let $S_{0}$ and $S_{1}$ be the two components of $h$ adjacent to $A$ such that $S_{1} \cap A=\partial_{r} S_{1}$. Then $S_{i}^{\prime}=U_{0} \cap S_{i}, i=0,1$, are the lids of $U_{0}$. Let $m$ be the least positive integer such that $\varphi^{m}\left(S_{1}\right)=S_{1}$; then $m$ is also the least positive integer such that $\varphi^{m}\left(S_{0}\right)=S_{0}$. For simplicity let $\psi=\varphi^{m}$.

Claim. For each $i$ (a) $S_{i} \neq B_{h}$, (b) $S_{i}^{\prime}$ is isotopic to $S_{i}$ in $\partial H$.

Proof. Suppose $S_{0}=B_{h}$. When $n=1$, it follows from the irreducibility of $\varphi \mid B_{h}$ (Lemma 2.5) that $S_{0}=S_{0}^{\prime}$, and hence $\varphi\left(U_{0}\right)=U_{0}$. This implies that $\varphi\left(S_{1}\right)=S_{1}$ and $p=\left|\partial B_{h}\right|=1$, a contradiction. When $n>1, \varphi \mid S_{0}=\mathrm{id}$, so that $\varphi \mid U_{0} \simeq$ id. By Lemma $5.18 \varphi \mid S_{0} \cup A \cup S_{1} \simeq$ id, contradicting Proposition 5.1, part 4. This proves (a). If some $S_{i}^{\prime}$ is not isotopic to $S_{i}$, then $S_{i}$ is a trivial component (Lemma 5.12, Proposition 5.1, part 5). Hence, $\psi \mid U_{0} \simeq$ id. A contradiction follows as above, so the claim is proved.

Proof of parts 1,2 . Let $s+1=\left|\left(\partial S_{0}-\partial_{r} S_{0}\right) / \psi\right|$ and $\widetilde{J}_{i}$ be the fibered knot in a homotopy sphere with $s$ unknotted holes with monodromy $\psi \mid\left(S_{i}, \partial_{r} S_{i}\right), i=$ 0,1 . Identify $U_{0}$ with $S_{1} \times I$ so that $S_{i} \cap U_{0}=S_{1} \times i$ for $i=0,1$ and $A=\partial_{r} S_{1} \times I$. Then by part 1 of Proposition $5.1 \psi \mid A=$ id. Isotop $\varphi$ in a neighborhood of $\partial\left(S_{0} \cup A \cup S_{1}\right)$ so that $\psi \mid S_{i} \cap U_{0}$ is the monodromy of $\tilde{J}_{i}$ for each $i$. The natural projection from $S_{1} \times 0$ to $-\left(S_{1} \times 1\right)$ gives a diffeomorphism $g: S_{0} \rightarrow-S_{1}$ such that (1) $g\left(\partial_{r} S_{0}\right) \neq \partial_{r} S_{1}$ and (2) 
$g \circ\left(\psi \mid S_{0}\right) \circ g^{-1} \simeq-\psi \mid S_{1}$ rel $\partial_{r} S_{1}$. Since $\partial_{*}=\partial_{r}$ by part 6 of Proposition 5.1, we have $\widetilde{J}_{1} \cong \widetilde{J}_{0}^{*}$, i.e., $S_{1}=S_{0}^{*}$.

Note that $\varphi$ permutes the set of handlebodies $\overline{H-\bigcup_{j} \varphi^{j}\left(U_{0}\right)}$. Let $H_{i}, 0 \leq$ $i \leq s$, be representatives of the orbits such that $B_{h} \subset H_{0}$. Then $F_{1} \cap H_{i}$ consists of two components for each $i>0$. For each $0 \leq i \leq s$ let $T_{i}$ be the component of $F_{1} \cap H_{i}$ such that $T_{i} \cap S_{1} \neq \varnothing$. For $1 \leq i \leq s$ let $T_{s+i}$ be $\left(F_{1} \cap H_{i}\right)-T_{i}$ (Figure 5). Let $m_{i}(1 \leq i \leq s)$ be the least positive integers such that $\varphi^{m_{i}}\left(H_{i}\right)=H_{i}$, and let $m_{0}=m$. For $0 \leq i \leq 2 s$ define $k_{i}$ to be the (possibly composite) fibered knot in a homotopy sphere with monodromy $\varphi^{m_{i}} \mid T_{i}$. Define $J_{1}$ to be the fibered knot in a homotopy solid torus with monodromy $\varphi \mid\left(\overline{F_{1} \cap H_{0}-\bigcup \varphi^{j}\left(T_{0}\right)}, \partial F_{1}\right)$. It follows from $S_{i} \neq B_{h}$ that when $n=1$, the fiber of $J_{1}$ is not an annulus. Hence, $J_{1}\left(k_{0}\right)$ is a $(p, q)$ cable of a nontrivial knot when $n=1$. Now $K_{1}$ has a satellite presentation

$$
K_{1}=J_{1}\left(\widetilde{J}_{0}\left(\tilde{J}_{0}^{*}\left(k_{0}, \ldots, k_{s}\right), k_{s+1}, \ldots, k_{2 s}\right)\right) \text {. }
$$

By Lemma 2.2 we may assume $\varphi^{m_{i}} \mid \operatorname{Fr} H_{i}=$ id. Hence $\varphi^{m_{i}} \mid \partial H_{i}, i \geq 1$, is the closed monodromy of $k_{i} \# k_{s+i}$, which is $\geq 0$. Also $\varphi \mid \partial H_{0}$ is the closed monodromy of $K^{\prime}=J_{1}\left(k_{0}\right) \# K_{2} \# \cdots \# K_{n}$, which is $\geq 0$. Let $F_{1}^{\prime} \subset \partial H_{0}$ be the fiber of $J_{1}\left(k_{0}\right)$, i.e., the component of $\overline{\partial H_{0}-\widehat{D}_{n}}$ containing $T_{i}$ for all $i$. By Lemma 5.14 the core of each component of the $m$ annuli Fr $H_{0}$ is primitive for $\overline{H-H_{0}}$, which consists of $m$ handlebodies. Hence, a regular neighborhood of $F_{1}^{\prime} \cup \overline{H-H_{0}}$ in $H$ is a compression body with $\partial_{-} \cong F_{1}^{\prime}, \partial_{+} \cong F_{1}$. This compression body induces $K_{1} \geq J_{1}\left(k_{0}\right)$, so $K_{1}^{\prime}=J_{1}\left(k_{0}\right)$ is the desired knot in part 2.

Let us show the second statement of part 1. Since $\alpha\left(k_{i} \# k_{s+i}\right)<\alpha(K)$, we can apply Theorem 5.3 to $k_{i} \# k_{s+i}$ for each $i$. Although $k_{i}, k_{s+i}$ may not be prime

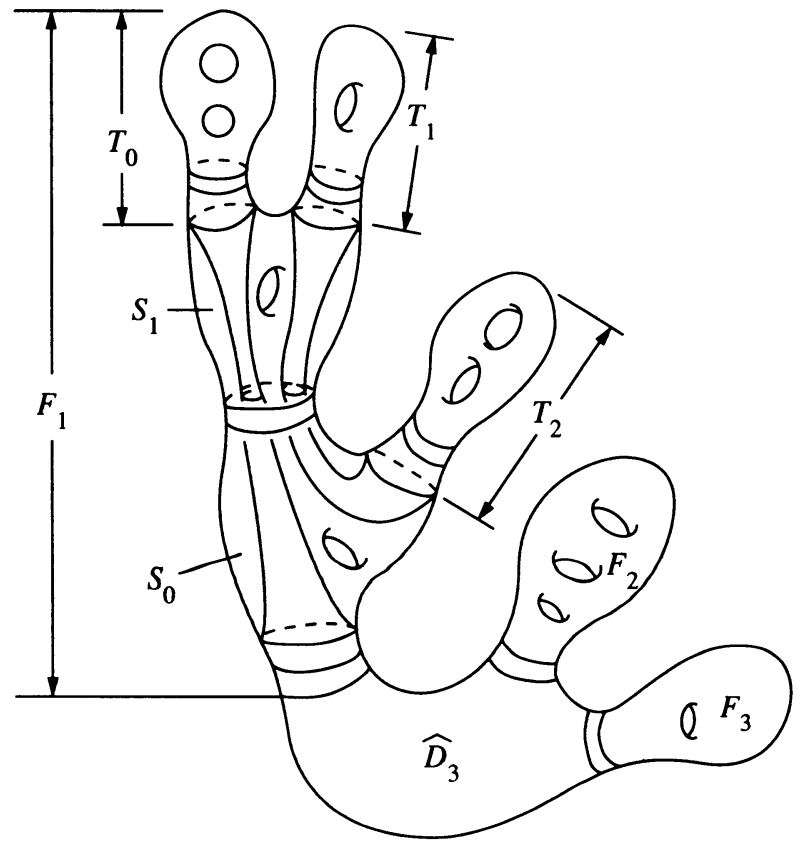

FiguRE 5. $s=m=m_{1}=1$ and the fiber of $J_{1}$ is an annulus 
knots, it follows that (1) $k_{i} \cong-k_{s+i}$ or (2) at least one of these knots has a satellite presentation in Lemma 5.19, part 1. (2) implies that $K_{1}$ also has such a presentation. If (1) is the case, let $J$ be the fibered knot whose monodromy is the restriction of $\psi$ to $\left(S_{0} \cup\left(\bigcup_{j} \bigcup_{i=s+1}^{2 s} \varphi^{j}\left(T_{i}\right)\right), \partial_{r} S_{0}\right)$, a surface with two boundary components. Then $K_{1}=J_{1}\left(J_{(}\left(J^{*}\left(k_{0}\right)\right)\right)$ as stated in part 1 .

Proof of parts 3, 4. Write $H^{\prime}=H_{K^{\prime}}\left(=H_{0}\right.$ in the proof of part 2). Let us consider the boundary pattern $h_{K^{\prime}}$. It is clear that every component of $h_{K}$ in $F_{i}, i>1$, is contained in $h_{K^{\prime}}$, and hence incompressible in $H^{\prime}$. To go further choose $G_{0}, G_{1} \in h_{K} \cap H^{\prime}$ as follows. Let $G_{1}$ be the nonannulus component of $h_{K} \cap H^{\prime}$ such that $\partial_{r} G_{1}$ is parallel to (a component of) $\partial S_{1}$ in $\partial H$. If $B_{h}$ is an annulus and $\partial_{r} S_{0} \subset B_{h}$, let $G_{0}=B_{h}$. Otherwise, let $G_{0}$ be the nonannulus component of $h_{K} \cap H^{\prime}$ such that $\partial_{r} S_{0}$ is parallel to $\partial G_{0}$. Let $A^{\prime}$ be the annulus in $\partial H^{\prime}$ bounded by $G_{1}$ and $G_{0}$. Note that $\psi \mid A^{\prime}=$ id. Let $k$ be the least positive integer such that $\varphi^{k}\left(G_{0}\right)=G_{0}$. Let $T=G_{0} \cup \bigcup_{i} \varphi^{k i}\left(A^{\prime} \cup G_{1}\right)$. Note that $T$ is connected, and either $\varphi^{i}(T)=T$ or $\varphi^{i}(T) \cap T=\varnothing$ for each $i$. Each component of $h_{K}$ in $\overline{\partial H^{\prime}-\bigcup_{i} \varphi^{i}(T)}$ is a component of $h_{K^{\prime}}$ and incompressible in $H^{\prime}$. Let us determine $h_{K^{\prime}} \cap T$.

Case 1. $T \notin h_{K^{\prime}}$. If $G_{0}$ is an annulus, then $h_{K^{\prime}} \cap T$ consists of $G_{0}$ and the components of $\varphi^{i}\left(A^{\prime} \cup G_{1}\right), i \geq 0$. Otherwise, $h_{K^{\prime}} \cap T$ consists of the components of $\varphi^{i}\left(G_{0}\right), \varphi^{i}\left(A^{\prime}\right), \varphi^{i}\left(G_{1}\right), i \geq 0$. It follows that $\alpha(K)>\alpha\left(K^{\prime}\right)$ and each nontrivial component of $h_{K^{\prime}}$ is incompressible in $H^{\prime}$.

Case 2. $T \in h_{K^{\prime}}$. We claim that $T$ is a trivial component of $h_{K^{\prime}}$. Since $G_{1}$ is not an annulus, the adjoint automorphism of $T, \varphi^{k} \mid T$, is reducible. Let $T^{\prime}$ be $T$ minus the disk bounded by $\partial F^{\prime}$ where $F^{\prime}\left(\subset \partial H^{\prime}\right)$ is the fiber of $K^{\prime}$. ( $T^{\prime}$ is possibly equal to $T$.) Since $\varphi^{k} \mid T$ is reducible, so is $\varphi^{k} \mid T^{\prime}$. Then, Lemma 3.3 part 5 implies that $\varphi^{k} \mid T^{\prime} \simeq \mathrm{id}$, so that $T$ is a trivial component of $h_{K^{\prime}}$. Hence, each nontrivial component of $h_{K^{\prime}}$ is incompressible in $H^{\prime}$ and $\alpha\left(K^{\prime}\right)<\alpha(K)$. This completes the proof of parts 3 and 4 .

Proof of Lemma 5.20. Let $K$ be a composite fibered knot in a homology sphere satisfying the assumption of Theorem 5.2. Suppose that $h_{K}$ is useful and some component $U_{0}$ of $U_{K}$ contains an annulus $A$ in $h_{K}-\left\{B_{h}\right\}$. If $A$ is not adjacent to $B_{h}$, then $\partial U_{0} \cap\left(\partial H_{K}-B_{h}\right)$ consists of the two lids of $U_{0}$ and (a nonempty set of) annuli connecting them. It follows that $\partial H_{K}-B_{h}$ is compressible in $H_{K}$, contradicting the assumption of Theorem 5.2, part 1 . Hence $A$ is adjacent to $B_{h}=\widehat{D}_{n}$, so that $\varphi \mid U_{0} \simeq$ id. By Lemma 5.18 this contradicts Proposition 5.1 part 4. Hence, $U_{K}$ contains no annulus components of $h_{K}-\left\{B_{h}\right\}$.

Let $K=K_{1} \# \ldots \# K_{n}$ be a fibered knot in a homotopy sphere satisfying the assumption of Theorem 5.3. Suppose that if $h_{K}$ is useful and $U_{K}$ contains no annulus component of $h_{K}-\left\{B_{h}\right\}$, then Theorem 5.3 holds for $K$. We prove Theorem 5.3 by induction on $\alpha(K)$ just as in the proof of Lemma 5.10. Without loss of generality assume that $h_{K}$ is useful and $U_{K}$ contains an annulus component of $h_{K} \cap F_{1}$ where $F_{1}$ is the fiber of $K_{1}$ in $\partial H_{K}$. Assume that Theorem 5.3 holds for every $L$ with $\alpha(L)<\alpha(K)$. Let $K_{1}^{\prime}$ be the knot obtained by applying Lemma 5.19, part 2 to $K$. Then $K^{\prime}=K_{1}^{\prime} \# K_{2} \# \cdots \# K_{n}$ satisfies the assumption of Theorem 5.3 and $\alpha\left(K^{\prime}\right)<\alpha(K)$. By the induction hypothesis Theorem 5.3 holds for $K^{\prime}$. Since $K_{1} \geq K_{1}^{\prime}$, this implies that Theorem 5.3, 
part 1 holds for $K$ even if $K_{1}^{\prime}$ is composite. Let us see that Theorem 5.3, part 2 holds for $K$. Assume that for some $i_{0}$ either $h_{K} \cap F_{i_{0}}$ or $K_{i_{0}}$ satisfies the assumption of part 2 of Theorem 5.3. It follows from Lemma 5.19, part 1 that $i_{0} \neq 1$. Then, applying Theorem 5.3, part 2 to $K^{\prime}$ proves that the theorem holds for $K$.

The reduction of Theorem 5.4 can also be proved by induction on $\alpha(K)$. We leave this to the reader as an exercise. This completes the proof of Lemma 5.20 .

5.3. Position of $U$ and $V(2)$. For a homotopically-ribbon fibered knot $K$ in a homology sphere, define $n, f, \varphi, H, h, U, V, B_{h}, \widehat{D}_{n}$ as in $\S 5.1$; in particular, $h$ is a useful boundary pattern. We do not necessarily assume that (1) $U$ contains no annulus components of $h-\left\{B_{h}\right\}$. We first show that if $n>2$, then (2) component of Fr $V$ is parallel to an annulus component of $h$. If $n>2$, assertions (1) and (2) contradict each other (Lemma 5.22). Hence the proof of Theorem 5.3 reduces to the case $n=2$, and the proof of Theorem 5.2 part 1 is completed. We then study the position of $\operatorname{Fr} V$ in $H$, which almost determines that of $\operatorname{Fr} U$ by Lemmas 5.16 and 5.17.

Let us recall one result of [19]. By the completeness of the characteristic submanifold $X, \overline{H-X}$ consists of simple 3-manifolds and (annuli) $\times I$ (cf. Example 3 of [19, p. 158]). An admissible automorphism of a simple 3-manifold is admissibly isotopic to a periodic map [19, Proposition 27.1].

Lemma 5.21. Let $\alpha$ be a component of $\operatorname{Fr} V$. If $\alpha$ is parallel to an annulus $A \in h$, then the following assertions 1 and 2 hold.

1. $\varphi^{k}$ preserves $S_{1} \cup A \cup S_{2}$ for some $k$ where $S_{1}(\neq) S_{2} \in h$ are adjacent to $A$, and $\varphi^{k} \mid S_{1} \cup A \cup S_{2}$ is isotopic to a periodic map.

2. Either $A=B_{h}$, or $A$ is adjacent to $B_{h}$ and $n=1$.

Proof. Let $V_{0}$ be the component of $V$ containing $\alpha$; then $V_{0}$ is a regular neighborhood of $A$ in $H$.

Claim. No component of $\partial(\operatorname{Fr} U)$ is parallel to a component of $\partial \alpha$ in $\partial H$.

Proof of Claim. Suppose that there is a component of $U, U_{0}$ say, such that some component of $\operatorname{Fr} U_{0}, \beta$ say, has a boundary component parallel to $\partial \alpha$ in $\partial H$. Then such a component of $\partial \beta$ is in $S_{1}$ or $S_{2}$. By Lemma 5.16 $\alpha$ and $\beta$ cobounds 3-manifold $W \cong \beta \times I$ in $H$. Now $U_{0} \cup W \cup V_{0}$ is an essential $I$-bundle in $(H, h)$ which cannot be admissibly isotoped into $X$, a contradiction. Claim is proved.

Let $\partial_{i} \alpha=\partial \alpha \cap S_{i}$ for $i=1,2$. Let $S_{i}^{\prime}$ be the component of $\overline{S_{i}-X}$ containing $\partial_{i} \alpha$. Then $S_{i}^{\prime}$ is not an annulus from Claim above and the fact that no component of $\partial(\operatorname{Fr} V)$ but $\partial_{2} \alpha$ is parallel to $\partial_{1} \alpha$. Set $S^{\prime}=S_{1}^{\prime} \cup \alpha \cup S_{2}^{\prime}$. Since $S^{\prime} \subset \overline{H-X}$ and $S_{i}^{\prime}$ is not an annulus, $S^{\prime}$ is contained in the boundary of a simple manifold component of $\overline{H-X}$. Hence, for some $k, \varphi^{k}\left(S^{\prime}\right)=S^{\prime}$ and $\varphi^{k} \mid S^{\prime}$ is isotopic to a periodic map leaving $S_{1}^{\prime}, \alpha$, and $S_{2}^{\prime}$ fixed as sets respectively. Let $A^{\prime}=V_{0} \cap \partial H$. Since $\alpha$ is parallel to $A^{\prime}$, the above statement about $\varphi^{k}$ remains true with $S^{\prime}$ (resp. $\alpha$ ) replaced by $S_{1}^{\prime} \cup A^{\prime} \cup S_{2}^{\prime}$ (resp. $A^{\prime}$ ). Lemma 5.18 then implies that $\varphi^{k} \mid S_{1} \cup A \cup S_{2}$ is isotopic to a periodic map as claimed in part 1.

Proposition 5.1, part 4 implies that $A=B_{h}$, or $n=1$ and $A$ is adjacent to $B_{h}$. This completes the proof of part 2 . 
Lemma 5.22. At least one of the following holds.

1. $U$ contains an annulus component of $h$.

2. Some component of $\operatorname{Fr} V$ is parallel to an annulus component of $h$.

Proof. Assume that neither 1 nor 2 above holds. Take a compressing disk $D$ for $\partial H$ in $H$ such that $|D \cap \operatorname{Fr} V|$ is minimal among all such disks. Let $\Delta$ be an outermost component of $D-\operatorname{Fr} V$ in $D$. If the disk $\Delta$ is contained in a component $V_{0}$, say, of $V$, then $\operatorname{Fr} V_{0}$ is an annulus parallel to $V_{0} \cap \partial H$. The annulus $V_{0} \cap \partial H$ is either a regular neighborhood of some annulus component of $h$ or contained in a component of $h$ (Lemma 5.15 part 3). The former implies assertion 2; the latter contradicts $\operatorname{Fr} V$ being essential in $(B, h)$. Hence $\Delta \cap$ Int $V=\varnothing$. Since assertion 1 is false, $V \cap \partial H$ contains all annulus components of $h$ (Lemma 5.17, part 1). It follows that the arc $\partial \Delta \cap \partial D$ is contained in some component of $h$, contradicting the fact that $\operatorname{Fr} V$ is essential.

Proposition 5.23. If $U_{K}$ contains no annulus components of $h_{K}-\left\{B_{h}\right\}$, then $n=1$ or 2 . In particular, the proof of Theorem 5.3 reduces to the case when $n=2, h_{K}$ is useful and $U_{K}$ contains no annulus components of $h_{K}-\left\{\widehat{D}_{2}\right\}$.

Proof. Suppose $n>2$; then $B_{h}$ is not an annulus. Thus, assertion 1 in Lemma 5.22 is not satisfied. Lemma 5.21 implies that assertion 2 is not satisfied. This contradicts Lemma 5.22.

The lemma below can be proved by a similar argument, so the proof is omitted.

Lemma 5.24. Suppose that $U$ contains no annulus components of $h-\left\{B_{h}\right\}$. If $n=2$ and $\widehat{D}_{2} \subset V$, then the component of $V$ containing $\widehat{D}_{2}$ is a regular neighborhood of $\widehat{D}_{2}$.

Proof of Theorem 5.2, part 1. The results follow from Lemma 5.20 and Proposition 5.23.

For a separating, simple loop $l$ in $\partial H$ let $[l]$ denote the (possibly empty) component of $\partial H-l$ which is disjoint from $B_{h}$. Let $l_{1}, l_{2}$ be essential separating simple loops in $\partial H$. We define a relation " $<$ " as follows: $l_{1}<l_{2}$ if $l_{i}$ is isotopic to some $l_{i}^{\prime}, i=1,2$, in $\partial H$ such that $l_{2}^{\prime} \subset\left[l_{1}^{\prime}\right]$.

Lemma 5.25. Let $A_{1}$ and $A_{2}$ be disjoint, essential, separating annuli in $H$. Let $\partial A=c_{1} \cup c_{2}$ and $\partial A_{2}=c_{3} \cup c_{4}$. If $c_{3}$ separates $c_{1}$ from $c_{2}$ in $\partial H$, then so does $c_{4}$. In particular, if $c_{1}<c_{3}<c_{2}$, then $c_{1}<c_{4}<c_{2}$.

Proof. Suppose for a contradiction that $c_{4}$ does not separate $c_{1}$ and $c_{2}$. Then there is an arc $a_{1}$ in $\partial H$ which connects $c_{1}$ and $c_{2}$, meets $c_{3}$ transversely in a single point, and $a_{1} \cap c_{4}=\varnothing$. Take an arc $a_{2}$ in $A_{1}$ with $\partial a_{1}=\partial a_{2}$ and set $c=a_{1} \cup a_{2}$. Push the simple loop $c$ slightly into Int $H$. Then $c$ meets the closed surface $S \cup A_{2}$ transversely in a single point, where $S$ is the submanifold of $\partial H$ bounded by $c_{3}$ and $c_{4}$. This is absurd, for $S \cup A_{2}$ and $c$ can be considered to be in $S^{3}$.

Assuming that $U$ contains no annulus components of $h-\left\{B_{h}\right\}$, introduce the following notation. Let $S$ be a nonannulus component of $h$. Let 


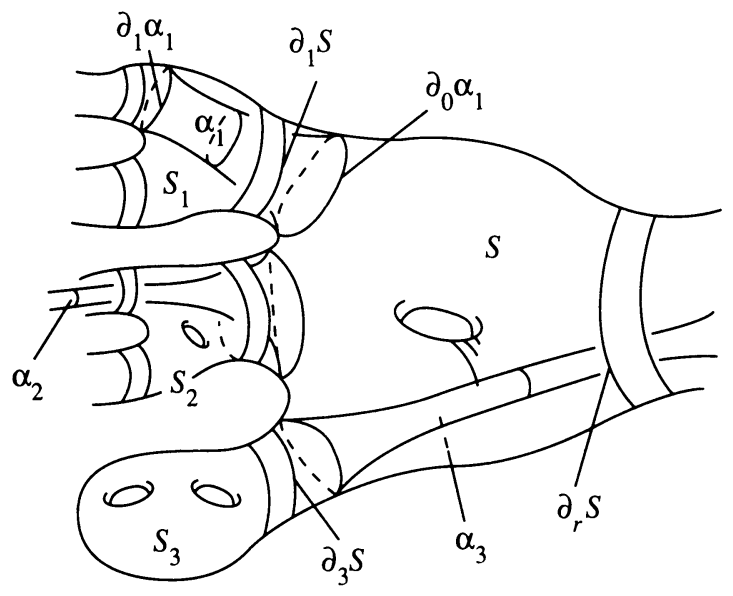

FIGURE 6

$\left\{\partial_{1} S, \ldots, \partial_{p} S\right\}$ denote the set of components of $\partial S$ if $S=B_{h}$, or the set of components of $\partial S-\partial_{r} S$ if $S \neq B_{h}$. For every $i, 1 \leq i \leq p$, there is exactly one component $\alpha_{i}$, say, of $\operatorname{Fr} V$ such that one component of $\partial \alpha_{i}$ is in $S$ and parallel to $\partial_{i} S$ (Lemmas 5.15 and 5.17). Let $\partial_{0} \alpha_{i}$ denote such a component, and $\partial_{1} \alpha_{i}$ the other component of $\partial \alpha_{i}$. We say that $\alpha_{i}$ is associated to $\partial_{i} S$. Let $S_{i}, 1 \leq i \leq p$, denote the nonannulus component of $h$ such that $\partial_{r} S_{i}$ is parallel to $\partial_{i} S$. See Figure 6 .

Lemma 5.26. Assume that (1) $U$ contains no annulus components of $h-\left\{B_{h}\right\}$, (2) either $S \neq B_{h}$ or no component of $\operatorname{Fr} V$ is parallel to an annulus in $h$. With the notation above the following hold.

1. There is no $i, 1 \leq i \leq p$, such that $\partial_{1} \alpha_{i}>\partial_{i} S$.

2. If $S=B_{h}$ (nonannulus), then $\partial_{1} \alpha_{i} \subset S$ for all $1 \leq i \leq p$.

Proof of part 1. For $S \in h$ let $N_{S}=\#\left\{G \in h-\{S\} \mid \partial_{r} G>\right.$ a component of $\left.\partial S\right\}$. We proceed by induction on $N_{S}$. Take an arbitrary nonannulus component $S \in h$. Suppose that Lemma 5:26 part 1 is true for any nonannulus component $S^{\prime} \in h$ such that $N_{S^{\prime}}<N_{S}$. Assume for a contradiction that $\partial_{1} \alpha_{i_{0}}>\partial_{i_{0}} S$ for some $i_{0}$. For simplicity set $\alpha=\alpha_{i_{0}}$. Suppose that $\partial_{1} \alpha$ is not parallel to $\partial_{0} \alpha$ in $\partial H$. Let $G_{1}$ be the component of $h$ containing $\partial_{1} \alpha$. There is a collection of nonannulus components of $h,\left\{G_{1}, \ldots, G_{s}, S\right\}$, such that $G_{j}$ is, via an annulus component of $h$, adjacent to $G_{j+1}$ if $j+1 \leq s$, and to $S$ if $j=s$ (thus $G_{s}=S_{i_{0}}$ ). Denote

$$
B=\left\{b \mid b \text { is a component of } \partial(\operatorname{Fr} V) \text { with } \partial_{0} \alpha<b<\partial_{1} \alpha\right\} .
$$

Using Lemma 5.25 repeatedly, we can find $b_{1}$ and $b_{2}$ in $B$ such that

1. $b_{1}<b_{2}$,

2. $b_{1} \cup b_{2}$ is a boundary of a component of $\operatorname{Fr} V$,

3. no element $b$ in $B$ satisfies $b_{1}<b<b_{2}$.

Let $\beta$ be the component of $\operatorname{Fr} V$ such that $\partial \beta=b_{1} \cup b_{2}$. Let $b_{1} \subset G_{\lambda}, b_{2} \subset$ $G_{\mu}$, where $1 \leq \lambda, \mu \leq s$. There are two possibilities about $\lambda$ and $\mu$.

Case 1. $\lambda \neq \mu$. It follows from Lemma 5.17 part 1 that $\mu=\lambda+1$ and $b_{1}$ is parallel to $b_{2}$ in $\partial H$. This contradicts either the lemma's assumption (2) or Lemma 5.21 part 2. Thus Case 1 does not occur. 


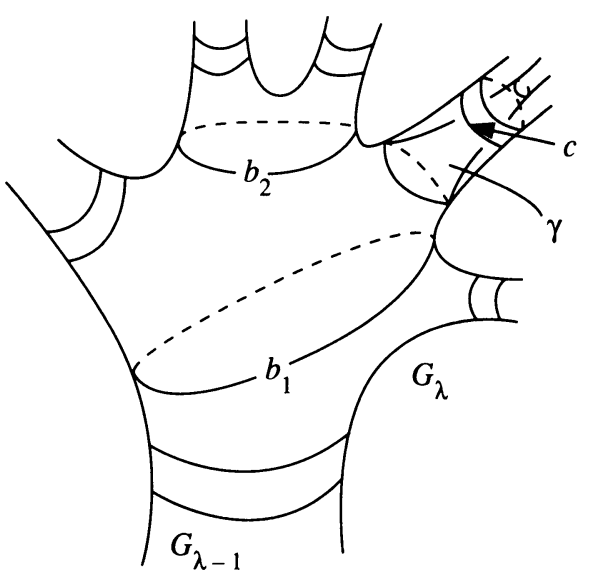

FIGURE 7

Case 2. $\lambda=\mu$.

Claim. There is a 3-submanifold $M$ of $H$ such that $M \cap \partial H \subset \operatorname{Int} G_{\lambda}$, Fr $M \subset \operatorname{Fr} V$, and $M \cap \partial H$ is connected.

Proof of Claim. Let $M_{1}$ be the closure of the outermost component of $H-\beta$, i.e., $M_{1} \cap \partial H$ is connected. If $\partial G_{\lambda} \cap M_{1}=\varnothing$, then $M_{1}$ is the desired manifold. So suppose not. Let $c$ be a component of $\partial G_{\lambda}$ contained in $M_{1}$. Since $c>b_{1}$, $c \neq \partial_{r} G_{\lambda}$. Let $\gamma$ be the component of $\operatorname{Fr} V$ associated to $c$, so $\partial_{0} \gamma\left(\subset \operatorname{Int} G_{\lambda}\right)$ is parallel to $c$ (Figure 7).

Since $N_{G_{\lambda}}<N_{S}$, by the induction hypothesis we can apply part 1 of Lemma 5.26 to $\partial_{1} \gamma$. It follows that $\partial_{1} \gamma \ngtr c$. Thus the 2-submanifold of $\partial H$ bounded by $\partial_{0} \gamma$ and $\partial_{1} \gamma$ contains $b_{1} \cup b_{2}$ but not $c$. Let $M_{2}$ be the closure of the outermost component of $M_{1}-\gamma$. Then $M_{2} \cap G_{\lambda}$ is nonempty, connected and does not contain $c$, so that $\left|M_{2} \cap \partial G_{\lambda}\right|<\left|M_{1} \cap \partial G_{\lambda}\right|$. If $M_{2} \cap \partial G_{\lambda}=\varnothing$, then $M_{2}$ is the claimed manifold $M$. Otherwise, construct $M_{3}$ from $M_{2}$ just as we obtain $M_{2}$ from $M_{1}$. In a finite number of steps we obtain the claimed 3-manifold $M$ in $H$.

However, the following lemma precludes the existence of this manifold. Thus Case 2 does not occur. Lemma 5.26, part 1 is proved.

Lemma 5.27. There is no 3-submanifold $M$ of $H$ such that $M \cap \partial H$ is connected and contained in a component of $h$, and $\operatorname{Fr} M \subset \operatorname{Fr} X$.

Proof. Apply Lemma 5.14 to $M$; then $M$ is a handlebody and the set of cores of $\operatorname{Fr} M$ is primitive for the handlebody $M$. Hence there is a 2 -faced disk $D$ in $M$ such that

$$
\begin{aligned}
D \cap \operatorname{Fr} M & =\text { a cocore of a component of } \operatorname{Fr} M, \\
D \cap \partial H & =\text { an arc in a component of } h .
\end{aligned}
$$

This implies that some component of $\operatorname{Fr} X$ is not essential in $(H, h)$, a contradiction.

Proof of Lemma 5.26, part 2. We proceed in a manner similar to Case 2 in the proof of Lemma 5.26, part 1. Define 3-submanifolds $M_{1}, M_{2}, \ldots$ of $H$ as follows, and finally obtain a 3-manifold violating Lemma 5.27. Let $M_{1}$ be the 
closure of the outermost component of $H-\alpha_{1}$. Let us see that $M_{1} \cap B_{h}$ is connected and $M_{1} \cap \partial_{1} B_{h}=\varnothing$. If $\partial_{1} \alpha_{1} \subset B_{h}$, this is obvious. So assume $\partial_{1} \alpha_{1} \subset \partial H-B_{h}$. Since $\partial B_{h}=\bigcup_{i=1}^{n} \partial_{i} B_{h}, \partial_{1} \alpha_{1}>\partial_{0} \alpha_{j}$ for some $1 \leq j \leq n$. By part 1 of Lemma $5.26 j \neq 1$. Thus, $M_{1} \cap B_{h}$ is connected and $M_{1} \cap$ $\partial_{1} B_{h}=\varnothing$, as desired. If $M_{1} \cap \partial B_{h}=\varnothing$, then $M_{1}$ is the desired manifold, i.e., $M_{1} \cap B_{h} \subset$ Int $B_{h}, M_{1} \cap B_{h}$ is connected and Fr $M_{1} \subset$ Fr $V$. Otherwise, there is a component of $\partial B_{h}$, say $\partial_{k} B_{h}(k \neq 1)$, contained in $M_{1} \cap B_{h}$. Let $M_{2}$ be the closure of the outermost component of $M_{1}-\alpha_{k}$. The same argument as above shows that $M_{2} \cap B_{h}$ is nonempty, connected and $M_{2} \cap \partial_{i} B_{h}=\varnothing, i=1, k$. Repeating this finally gives a 3-manifold $M$ contradicting Lemma 5.27.

Lemma 5.26 puts a strong restriction on the position of Fr $V$. Furthermore, we shall prove that there are no $i, j$ such that $\partial_{1} \alpha_{i}>\partial_{j} S$ for a knot in a homotopy sphere. In the next section this is done by using the Cyclic Surgery Theorem.

\section{Proof of Theorem 5.3}

Let $K_{i}, i=1,2$, be a prime fibered knot with fiber $F_{i}$ in a homotopy sphere $M_{i}$ such that $K_{1} \# K_{2} \geq 0$. For $K=K_{1} \# K_{2}$, define $f, \varphi, \widehat{D}_{2}, h, H, U, V$ as in $\S 5.1$. Throughout this section assume that $U$ contains no annulus components of $h-\left\{\widehat{D}_{2}\right\}$. By Proposition 5.23 the proof of Theorem 5.3 is reduced to proving Theorem 5.3 for our $K$. Therefore it suffices to show Proposition 6.1 below.

Proposition 6.1. With the above assumption the following hold.

1. There are (possibly composite or trivial) fibered knots $\widetilde{K}_{i}, i=1,2$, such that $K_{i} \geq \widetilde{K}_{i}$ and $\widetilde{K}_{1} \cong-\widetilde{K}_{2}$.

2. $\widetilde{K}_{i}$ is obtained from $K_{i}$ by successive reductions of type $J(k \#-k) \geq$ $J(0)$. In particular, if $K_{i}$ does not have a satellite presentation $J(k \#-k)$ where $k \not 0$, then $K_{i}=\widetilde{K}_{i}$.

Let $\mathscr{T}_{i}$ be a minimal collection of decomposing tori in $\overline{M_{i}-N\left(K_{i}\right)}$ in the sense of Jaco and Shalen, and Johannson. If $\mathscr{T}_{i} \neq \varnothing$, then denote by

$$
K_{i}=J_{i}\left(k_{1}^{i}, \ldots, k_{s_{i}}^{i}\right), \quad i=1,2,
$$

the satellite presentation of $K_{i}$ obtained via the subset of $\mathscr{T}_{i}$ consisting of all the tori closest to $K_{i}(\S 3)$. Note that each $k_{j}^{i}$ is nontrivial, but possibly composite. Let $Y_{i}$ denote the ambient manifold of $J_{i}$, so that $\left|\partial Y_{i}\right|=s_{i}$. For notational convenience if $\mathscr{T}_{i}=\varnothing$, denote $J_{i}=K_{i}, s_{i}=0$, and $Y_{i}=M_{i}$.

Then Proposition 6.1 follows from the proposition below.

Proposition 6.2. 1. $s_{1}=s_{2}$ and $\left(Y_{1}, J_{1}\right) \cong\left(-Y_{2},-J_{2}\right)$ as fibered knots.

2. There is a permutation $\sigma$ of $\left\{1, \ldots, s_{1}\right\}$ such that

(a) $k_{j}^{1} \# k_{\sigma(j)}^{2} \geq 0$ for $1 \leq j \leq s_{1}$.

(b) Set $k=k_{j}^{1} \# k_{\sigma(j)}^{2}$. Then $H_{k}$ is a submanifold of $H_{K}$ such that Fr $H_{k}$ consists of essential annuli, and $h_{k}-\left\{B_{h_{k}}\right\} \subset h_{K}-\left\{\widehat{D}_{2}\right\}$.

Proof of Proposition 6.1. We proceed, assuming Proposition 6.2. For simplicity let $s=s_{1}, \sigma(j)=j$ for all $1 \leq j \leq s$. Let $k_{j}^{i}=\#_{r=1}^{m(i)} L_{r}^{i}$ be the prime 
decomposition where $i=1,2$; then $\left(\#_{r=1}^{m(1)} L_{r}^{1}\right) \#\left(\#_{r=1}^{m(2)} L_{r}^{2}\right)=k \geq 0$. As in the proof of Lemma 5.10, compressing $B_{h_{k}}$ in $H_{k}$ yields a handlebody $H_{L}\left(\subset H_{k}\right)$ where $L=\#_{(i, r) \in S} L_{r}^{i}$ and $S \subset\{(i, r) \mid i=1,2,1 \leq r \leq m(i)\}$; then $|S|>1$ and $\left\{B_{h_{L}}\right\}=h_{L}-h_{k}$. By compressing $B_{h_{k}}$ maximally each component of $h_{L}$ is incompressible in $H_{L}$, and hence in $H_{K}$. If there is an essential $I$-bundle in $\left(H_{L}, h_{L}\right)$ containing an annulus in $h_{L}-\left\{B_{h_{L}}\right\}$, then $U_{K}$ contains an annulus in $h_{K}-\left\{\widehat{D}_{2}\right\}$, a contradiction. It follows that $U_{L}$ contains no annulus in $h_{L}-\left\{B_{h_{L}}\right\}$, so that $|S|=2$ by Proposition 5.23. Hence, $\left\{L_{r}^{i}\right\}_{i, r}$ can be paired into $\amalg\left\{L_{p}^{u}, L_{q}^{v}\right\}$ so that $L=L_{p}^{u} \# L_{q}^{v} \geq 0$ and $h_{L}$ is useful. We also see that $U_{L}$ does not contain any annulus in $h_{L}-\left\{\widehat{D}_{2}\right\}$.

Let us show Proposition 6.1 by induction on $\left|h_{K}\right|$. Note that for each pair $\left\{L_{p}^{u}, L_{q}^{v}\right\}$ we have $\left|h_{L}\right|<\left|h_{K}\right|$ where $L=L_{p}^{u} \# L_{q}^{v}$. Applying Proposition 6.1 to $L$, we obtain fibered knots $\widetilde{L_{p}^{u}}, \widetilde{L_{q}^{v}}$ such that $L_{p}^{u} \geq \widetilde{L_{p}^{u}}, L_{q}^{v} \geq \widetilde{L_{q}^{v}}$, and $\widetilde{L_{p}^{u}} \cong-\widetilde{L_{q}^{v}}$. Furthermore, $\widetilde{L_{p}^{u}}$ (resp. $\widetilde{L_{q}^{v}}$ ) is obtained from $L_{p}^{u}$ (resp. $L_{q}^{v}$ ) by successive reductions of type $J(k \#-k) \geq J(0)$. It follows that $k_{j}^{i} \geq \#_{r=1}^{m(i)} \widetilde{L_{r}^{i}}$ for $i=1,2$. For $i=1,2$ let $N_{i}$ be a (possibly empty) subset of $\{1, \ldots, m(i)\}$ which is minimal among all subsets $N_{i}^{\prime}$ such that $\{1, \ldots, m(i)\}-N_{i}^{\prime}$ can be paired into $\coprod_{t=1}^{m}\left\{p_{t}, q_{t}\right\}$ with $\widetilde{L_{p_{t}}^{i}} \cong-\widetilde{L_{q_{t}}^{i}}$. Recalling that $L_{r}^{i}, \widetilde{L_{r}^{i}}$ and $N_{i}$ depend on $j$, define $\tilde{k_{j}^{i}}$ for $i=1,2,1 \leq j \leq s$, as follows.

$$
\tilde{k}_{j}^{i}= \begin{cases}\underset{r \in N_{i}}{\#} \widetilde{L_{r}^{i}} & \text { if } N_{i} \neq \varnothing, \\ 0, \text { the unknot in } S^{3} & \text { if } N_{i}=\varnothing .\end{cases}
$$

Then $\tilde{k_{j}^{1}} \cong-\tilde{k_{j}^{2}}$, and $\tilde{k_{j}^{i}}$ is obtained from $k_{j}^{i}$ by successive reductions of type $J(k \#-k) \geq J(0)$. Therefore, $J_{i}\left(\tilde{k}_{1}^{i}, \ldots, \tilde{k}_{s}^{i}\right)=\widetilde{K}_{i}$ (say) is also obtained from $K_{i}$ by such reductions, and $\widetilde{K}_{1} \cong-\widetilde{K}_{2}$. This establishes Proposition 6.1.

Proof of Proposition 6.2. Let $h=h_{K}$. Let $R$ (resp. $S$ ) be the component of $h$ which is adjacent to $\widehat{D}_{2}$ and contained in $F_{1}$ (resp. $F_{2}$ ), a fiber of $K_{1}$ (resp. $\left.F_{2}\right)$. Let $\partial_{i} R, 1 \leq i \leq p_{1}$ (resp. $\partial_{j} S, 1 \leq j \leq p_{2}$ ) be the components of $\partial R-\partial_{r} R$ (resp. $\partial S-\partial_{r} S$ ). Let $R_{i}, 1 \leq i \leq p_{1}$ (resp. $\left.S_{j}, 1 \leq j \leq p_{2}\right)$ be the components of $h$ such that $\partial_{r} R_{i}$ (resp. $\partial_{r} S_{j}$ ) is parallel to $\partial_{i} R$ (resp. $\partial_{j} S$ ) in $\partial H$. We denote by $\alpha_{i}, 1 \leq i \leq p_{1}$ (resp. $\beta_{j}, 1 \leq j \leq p_{2}$ ) the components of Fr $V$ associated to $\partial_{i} R$ (resp. $\partial_{i} S$ ). Note that $s_{1}$ (resp. $s_{2}$ ) is equal to the number of orbits $\left|\left\{\partial_{i} R\right\}_{i} / \varphi\right|$ (resp. $\left|\left\{\partial_{j} S\right\}_{j} / \varphi\right|$ ).

Denote by $A_{i}$ (resp. $B_{j}$ ) the annulus in $\partial H$ bounded by $\partial_{i} R$ and $\partial_{0} \alpha_{i}$ (resp. $\partial_{j} S$ and $\left.\partial_{0} \beta_{j}\right)$. Isotop $\varphi \operatorname{rel} \partial H-R \bigcup S$ so that $\varphi \mid \overline{R-\bigcup A_{i}}\left(\operatorname{resp} . \varphi \mid \overline{S-\bigcup B_{j}}\right)$ is the monodromy of $J_{1}$ (resp. $\left.J_{2}\right)$. Then, if $\varphi^{m}\left(A_{i}\right)=A_{i}$ for some $m$, then $\varphi^{m} \mid A_{i}=$ id (the same is true of $B_{j}$ ).

We divide the proof into the two case $\widehat{D}_{2} \subset U$ and $\widehat{D}_{2} \subset V$. Note that $\varphi \mid R$ and $\varphi \mid S$ are irreducible.

Case 1. $\widehat{D}_{2} \subset U$. Let $U_{0}$ be the component of $U$ containing $\widehat{D}_{2}$. Then $R \cap U_{0}$ and $S \cap U_{0}$ are the lids of $U_{0}$. By Lemma 5.16 Fr $U_{0}$ is parallel to $\bigcup \alpha_{i}$. Isotop Fr $U_{0}$ to $\bigcup \alpha_{i}$; then $R \cap U_{0}=\overline{R-\bigcup A_{i}}, S \cap U_{0}=\overline{S-\bigcup B_{j}}$. Identify $U_{0}$ with $R \times I$ such that $R \cap U_{0}=R \times 0, S \cap U_{0}=R \times 1$, and $\widehat{D}_{2}=\partial_{r} R \times I$. 
Then $\varphi \mid U_{0}$ gives a diffeomorphism of $R \times I$ such that $\varphi \mid\left(R, \partial_{r} R\right) \times\{i\}$ is the monodromy of $J_{i+1}, i=0,1$, and $\varphi \mid \partial_{r} R \times I=$ id. Since $\partial_{r} S=\partial_{r} R \times 1$, Lemma 2.1 shows that $\left(Y_{1}, J_{1}\right) \cong\left(-Y_{2},-J_{2}\right)$ as fibered knots and $s_{1}=s_{2}$. This completes the proof of Proposition 6.2, part 1.

Note that there is a permutation $\rho$ of $\left\{1, \ldots, p_{1}\right\}$ such that $\alpha_{i}=\beta_{\rho(i)}$. By the action of $\varphi$ on $\left\{\alpha_{i}\right\}_{i}, \rho$ induces the permutation $\sigma$, say, of $\left\{1, \ldots, s_{1}\right\}$. For simplicity assume that $\sigma$ and $\rho$ are the identities. Take any $i$ such that $1 \leq i \leq s_{1}$, and let $m$ be the least positive integer such that $\varphi^{m}\left(R_{i}\right)=R_{i}$. Let $H^{\prime}$ be the closure of the component of $H-\alpha_{i}$ containing $R_{i}$. By Lemma $2.2 \varphi^{m} \mid \alpha_{i} \simeq \operatorname{idrel} \partial \alpha_{i}$. Thus $\varphi^{m} \mid \partial H^{\prime}$ is the closed monodromy of $k_{i}^{1} \# k_{i}^{2}=$ $k$. Lastly consider part 2(b). Let $A$ be the annulus on $\partial H^{\prime}$ bounded by $R_{i}$ and $S_{i}$. If $k_{i}^{1}$ (resp. $k_{i}^{2}$ ) is a composite knot, then $R_{i}$ (resp. $S_{i}$ ) is a trivial component of $h_{K}$. Thus the base, $B$, of $h_{k}$ is equal to either $A, R_{i} \cup A, A \cup S_{i}$, or $R_{i} \cup A \cup S_{i}$, so that $\{B\}=h_{k}-h_{K}$, completing the proof in Case 1.

Case 2. $\widehat{D}_{2} \subset V$. Let $V_{0}$ be the component of $V$ containing $\widehat{D}_{2}$. By Lemma $5.24 V_{0}$ is a regular neighborhood of $\widehat{D}_{2}$. Then $g=\varphi \mid R \cup \widehat{D}_{2} \cup S$ is admissibly isotopic to a periodic map (Lemma 5.21). It follows from Lemma 2.3 that $J_{i}$ is a torus knot in $S^{3}$ or a cable of $S^{1} \times D^{2}$ of type $\left(p_{i}, q_{i}\right)$, say, where $p_{1}, p_{2}>1$. In what follows we suppose that $J_{1}$ is the $\left(p_{1}, q_{1}\right)$ cable of $S^{1} \times D^{2}$. The case when both of $J_{i}$ are torus knots is considered at the end of Subcase 1 below. Lemma 2.4 implies that $g^{p_{1} q_{1} p_{2} q_{2}}$ is isotopic to a $\left(p_{1} q_{1}+p_{2} q_{2}\right)$-Dehn twist along a core of $\widehat{D}_{2}$. Hence $(*) p_{1} q_{1}+p_{2} q_{2}=0$, for $g$ is periodic up to isotopy. Consider the position of $\partial_{1} \alpha_{1}$. Note that $\varphi$ permutes $\left\{\partial_{i} R\right\}_{i=1}^{p_{1}}$ cyclically and hence $s_{1}=1$. If $\partial_{1} \alpha_{1}>\partial_{i} R$ for some $i \neq 1$, then $\partial_{1} \alpha_{i}>\partial_{j} R$ for some $j \neq i$, and $\partial_{j} R$ separates $\partial_{1} \alpha_{i}$ from $\partial_{0} \alpha_{i}$. This is impossible. Hence, by Lemma 5.26, part $1 \partial_{1} \alpha_{1} \subset R$ or $\subset F_{2}$.

Subcase 1. $\partial_{1} \alpha_{1} \subset F_{2}$. If $\partial_{1} \alpha_{1} \subset F_{2}-S$, then $\partial_{j} S<\partial_{1} \alpha_{1}$ for some $j, 1 \leq$ $j \leq p_{2}$. Since $\partial_{0} \beta_{j}$ separates $\partial_{0} \alpha_{1}$ from $\partial_{1} \alpha_{1}$, so does $\partial_{1} \beta_{j}$. Thus $\partial_{1} \beta_{j}$ is parallel to $\partial_{r} S$. This contradicts Lemma 5.24, hence $\partial_{1} \alpha_{1} \subset S$. Therefore, $\partial_{1} \alpha_{1}=\partial_{0} \beta_{j}$ for some $1 \leq j \leq p_{2}$. This is true of each $\partial_{1} \alpha_{i}, 1 \leq i \leq p_{1}$, therefore there is a permutation $\rho$ of $\left\{1, \ldots, p_{1}\right\}$ such that $\alpha_{i}=\beta_{\rho(i)}$. It follows that $p_{1}=p_{2}$. Recalling $(*)$, we obtain $q_{1}=-q_{2}$ and hence $J_{1} \cong-J_{2}$. This proves Proposition 6.2, part 1.

For simplicity set $p_{1}=p, q_{1}=q$. Let $M$ be a submanifold of $H$ such that $\partial M=\bigcup \alpha_{i} \cup \overline{R-\bigcup A_{i}} \cup \widehat{D}_{2} \cup \overline{S-\bigcup B_{i}}$. Then $M$ is a simple manifold, so that $\varphi \mid M: M \rightarrow M$ is admissibly isotopic to a periodic map. In particular, $\varphi^{p q} \mid \partial M$ is periodic. By Lemma $2.4 \varphi^{p q} \mid M \cap \partial H$ is isotopic rel $\bigcup \partial \alpha_{i}$ to the product of $\tau_{i}^{p}, \eta_{i}^{-p}, 1 \leq i \leq p$, where $\tau_{i}$ (resp. $\eta_{i}$ ) is a Dehn twist along a simple loop parallel to $\partial_{0} \alpha_{i}$ (resp. $\left.\partial_{1} \alpha_{i}\right)$. Hence $\varphi^{p} \mid \alpha_{i} \simeq \operatorname{id} \operatorname{rel} \partial \alpha_{i}$ for each $i$. The proof of Proposition 6.2 part 2 is now completed by the argument similar to the last paragraph in Case 1. [If both $J_{i}$ are torus knots, then $(H, h)$ is a simple manifold, so that $\varphi$ is periodic up to isotopy. By considering the fixed point data of $\varphi \mid \partial H$ we obtain $J_{1} \cong-J_{2}$.]

Subcase 2. $\partial_{1} \alpha_{1} \subset R$. We shall show that Subcase 2 is not the case. To do this we shall need the Cyclic Surgery Theorem. Note that $\partial_{1} \alpha_{1}=\partial_{0} \alpha_{i}$ for some $1<i \leq p_{1}$, i.e., $\alpha_{1}=\alpha_{i}$. It follows that $\left\{\partial_{0} \alpha_{1}, \ldots, \partial_{0} \alpha_{p_{1}}\right\}$ are paired via the annuli $\alpha_{j}$. Therefore, $p_{1}$ is even. Without loss of generality assume that $\alpha_{1}=\alpha_{2}$. Set $p=p_{1}, q=q_{1}$, and $\psi=\varphi^{p}$. First isotop $\varphi$ rel $\partial H-R$ 


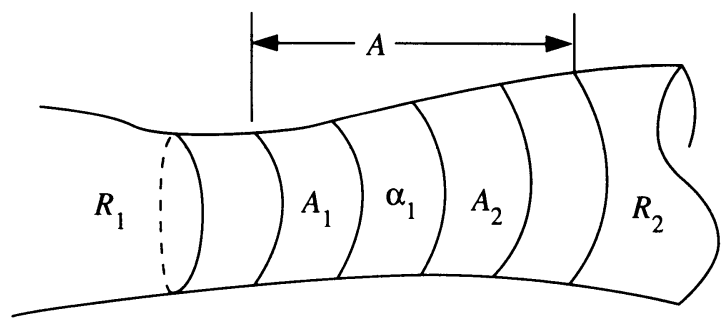

FIGURE 8

so that $\varphi$ satisfies the assertions (a)-(e) of Lemma 2.4, part 2 with $N_{i}, i>0$, replaced by $A_{i}, \quad 1 \leq i \leq p_{1}$. Then $\varphi \mid \overline{R-N_{0} \cup\left(\bigcup A_{i}\right)}$ is a periodic map of order $|p q|$, and $\psi \mid A_{i}$ is a $-p / q$-Dehn twist from $\partial_{i} R$ to $\partial_{0} \alpha_{i}$.

Claim 1. $\psi \mid \alpha_{1} \simeq$ id rel $\partial \alpha_{1}$.

Proof. Let $T=\overline{R-N_{0} \cup\left(\bigcup A_{i}\right)} \cup\left(\bigcup \alpha_{i}\right)$, a surface with one puncture. Since $T \subset \overline{H-X}, \psi \mid T: T \rightarrow T$ is isotopic to a periodic map. Since $\psi \mid \overline{T-\bigcup \alpha_{i}}$ is periodic, $\psi \mid \alpha_{1}$ is isotopic rel $\partial \alpha_{1}$ to a periodic map. Hence the rotational angle of $\psi \mid \partial_{0} \alpha_{1}$ is equal to that of $\psi \mid \partial_{1} \alpha_{1}$. It follows that $p / q \equiv-p / q$ $(\bmod 1)$, thus $|q|=1,2$. Since $p$ is even, we have $q= \pm 1$. This implies that $\psi \mid \partial_{0} \alpha_{1}=$ id, so that $\psi \mid \alpha_{1}=$ id. Claim 1 is proved.

Let $H^{\prime}$ be the closure of the component of $H-\alpha_{1}$ containing $R_{1}$ and $R_{2}$. Let $A$ be the annulus in $\partial H^{\prime}$ bounded by $\partial_{1} R$ and $\partial_{r} R_{2}$. Since $A \supset$ $A_{1} \cup A_{2}, \psi \mid A$ is a $-2 p / q$-Dehn (full-)twist (Figure 8). Let $F_{i}^{\prime}, i=1,2$, be the components of $\overline{\partial H^{\prime}-A}$ containing $R_{i}$. Recall that $K_{1}=J_{1}\left(k_{1}^{1}\right)$ and $\psi \mid F_{i}^{\prime}$ is the monodromy of $k_{1}^{1}$ for each $i$. For simplicity let $k_{1}=k_{1}^{1}$. Then $\psi \mid A \cup F_{2}^{\prime}$ is the monodromy of a fibered knot $k_{1}^{\prime}$ in a homology sphere defined as follows. Let $N$ be the ambient manifold of $k_{1}$, and let $N^{\prime}$ be the homology sphere obtained from $N$ after a $2 p / q$-Dehn surgery along $k_{1}$. The knot $k_{1}^{\prime}$ in $N^{\prime}$ is the core of the glued solid torus. Thus $\psi \mid \partial H^{\prime}$ is the closed monodromy of $K^{\prime}=k_{1} \# k_{1}^{\prime}$, a knot in the homology sphere $N \# N^{\prime}$.

Claim 2. $k_{1}=k_{1}^{1}$ is not prime.

Proof. Suppose for a contradiction that $k_{1}$ is prime. Then the boundary pattern $h_{K^{\prime}}$ for $H^{\prime}$ is

$$
h_{K^{\prime}}=\left\{A \cup R_{2}, \text { the components of } h_{K} \cap \overline{\partial H^{\prime}-A \cup R_{2}}\right\} .
$$

Here the annulus component of $h_{K^{\prime}}$ bounded by $R_{1}$ and $A$ plays the role of $\widehat{D}_{2}$. We call this annulus $\widetilde{D}_{2}$. The usefulness of $h_{K}$ for $H$ guarantees that $h_{K^{\prime}}$ is a useful boundary pattern for $H^{\prime}$. We first show that $U_{K^{\prime}}$ contains no annulus components of $h_{K^{\prime}}-\left\{\widetilde{D}_{2}\right\}$. If there is such a component, $U^{\prime}$, of $U_{K^{\prime}}$, then $U^{\prime}$ is not a component of $U_{K}$. Therefore $U^{\prime}$ intersects a component of $h_{K^{\prime}}$ which does not belong to $h_{K}$, so that $\left(A \cup R_{2}\right) \cap U^{\prime}$ is a lid of $U^{\prime}$. However, $U^{\prime}$ is disjoint from $\widetilde{D}_{2}$ which is adjacent to $A$. Since $A$ is an annulus, this implies that $U^{\prime}$ can be admissibly isotoped off $A$. Thus $U^{\prime}$ is an essential $I$-bundle in $\left(H_{K}, h_{K}\right)$. Then $U^{\prime}$ is admissibly isotoped to some component of $U_{K}$, a contradiction. Therefore, $U_{K^{\prime}}$ contains no annulus in $h_{K^{\prime}}-\left\{\widetilde{D}_{2}\right\}$.

Let us show $\widetilde{D}_{2} \subset U_{K^{\prime}}$. Suppose not. As in the beginning of Case 2, Lemmas 5.24 and 5.21 imply that $\psi \mid T$ is isotopic to a periodic map where $T=R_{1} \cup$ $\widetilde{D}_{2} \cup A \cup R_{2}, \psi \mid R_{1}$ and $\psi \mid R_{2}$ are isotopic to the same periodic map of order 
$m$, say. Then $\psi^{m} \mid T \simeq \tau^{2-2 m p / q}$, where $\tau$ is a Dehn twist along the core of $A$. Since $q \neq m p$ and the core of $A$ is not parallel to $\partial T, \psi^{m} \mid T$ is not isotopic to a periodic map, a contradiction. Hence $\widetilde{D}_{2} \subset U_{K^{\prime}}$. Let $U_{0}$ be the component of $U_{K^{\prime}}$ containing $\widetilde{D}_{2}$. Following the argument in Case 1 , identity $U_{0}$ with $R_{1} \times I$ such that $U_{0} \cap R_{1}=R_{1} \times 0, U_{0} \cap\left(A \cup R_{2}\right)=R_{1} \times 1$, and $\widetilde{D}_{2}=\partial_{r} R_{1} \times I$. The natural projection $R_{1} \times 0 \rightarrow-\left(R_{1} \times 1\right)$, then, induces a diffeomorphism $g: R_{1} \rightarrow-R_{2}$ such that

$$
g^{-1} \circ\left(\psi \mid-R_{2}\right) \circ g=\tau^{2 p / q} \circ\left(\psi \mid R_{1}\right)
$$

where $\tau: R_{1} \rightarrow R_{1}$ is a Dehn twist along a simple loop parallel to $\partial_{r} R_{1}$.

Let $J$ be the fibered knot in a homotopy sphere with unknotted holes, $P$, with monodromy $\psi \mid R_{1}$. Set $M=\overline{P-N(J)}$, which is not $S^{1} \times D^{2}$. Then $g$ induces a diffeomorphism $g^{\prime}: M \rightarrow-M$ such that $g^{\prime}(\mu)=\mu \lambda^{r}, g^{\prime}(\lambda)=\lambda^{-1}$, where $r=-2 p / q$ and $(\mu, \lambda)$ is a pair of a meridian and a longitude of $J$. It follows that the Dehn filling $-M\left(\mu \lambda^{r}\right) \cong M(\mu)=P$. Since $p>1$ and $q= \pm 1$, $|r| \geq 4$. If $P$ is a homotopy sphere, then by the Cyclic Surgery Theorem [6] $M$ is a Seifert fibered manifold. It follows that $P=S^{3}$ and $J$ is a nontrivial torus knot. Since torus knots have Property $\mathrm{P}, M\left(\mu \lambda^{r}\right) \not S^{3}$, a contradiction. Hence $\partial P \neq \varnothing$. Then $\partial P$ compresses in $M\left(\mu \lambda^{r}\right)$ and $M(\mu)$. By [6, Theorem 2.4.4] $M\left(\neq T^{2} \times I\right)$ is a cable space. Thus, $P=S^{1} \times D^{2}$ and $J$ is a nontrivial cable of $P$. However, this contradicts the fact that $M\left(\mu \lambda^{r}\right)=S^{1} \times D^{2}[12$, Lemma 7.2]. Claim 2 is proved.

Claim 3. $k_{1}$ is not a composite knot.

Proof. Recall that $K_{1}=J_{1}\left(k_{1}\right)$ and $K^{\prime}=k_{1} \# k_{1}^{\prime}$. Assume for a contradiction that $k_{1}$ is composite; $\psi \mid R_{1}=$ id and $R_{1}$ is a punctured disk with $\left|\partial R_{1}\right|>2$. Then the boundary pattern $h_{K^{\prime}}$ for $H^{\prime}$ is the same as the one when $k_{1}$ is prime. That is,

$$
h_{K^{\prime}}=\left\{A \cup R_{2} \text {, the components of } h_{K} \cap \overline{\partial H^{\prime}-A \cup R_{2}}\right\} .
$$

This time $R_{1}$ plays the role of $\widehat{D}_{l}$ where $l$ is the number of factors in the prime decomposition of $K^{\prime}$. It follows that $l>2$. The usefulness of $h_{K}$ shows that $h_{K^{\prime}}$ is useful for $H^{\prime}$. By Proposition 5.23 $U_{K^{\prime}}$ contains an annulus component of $h_{K^{\prime}}$. The argument about $U^{\prime}$ in Claim 2 shows that $U_{K^{\prime}}$ contains the annulus in $h_{K^{\prime}}$ bounded by $A$ and $R_{1}$. However, $U_{K^{\prime}}$ does not contain an annulus component adjacent to a trivial component, a contradiction.

Since Claims 2 and 3 contradict each other, Subcase $2\left(\partial_{1} \alpha_{1} \subset R\right)$ is impossible. This completes the proof of Proposition 6.2.

\section{Proof of Theorem 5.4}

Let $K=J(k)$ be a fibered knot in a homotopy sphere such that $K \geq 0$, $k \not 00$, and $J$ is the $(p, q)$ cable of $S^{1} \times D^{2}$ with $p>1$. As in Theorem 5.4 assume that each nontrivial component of $h_{K}$ is incompressible in $H_{K}$. By Lemma 5.20 it suffices to deduce a contradiction by assuming that $U_{K}$ contains no annuli in $h_{K}-\left\{B_{h}\right\}$. Keep in mind that $B_{h}$ is the union of the fiber of $J$ and a disk. Because a boundary irreducible Seifert fibered manifold in the exterior of $K$ is a cable space or (a punctured disk) $\times S^{1}$ [24, Lemma 2.1].

Case 1. No component of $V$ is a regular neighborhood of an annulus in $h_{K}-\left\{B_{h}\right\}$. 
As in the proof of Proposition 5.23, we can deduce a contradiction by assuming that $\left|B_{h}\right|>2$. Hence, $K$ is the $(2, \pm 1)$ cable of $k$. Note that $\hat{f}^{2} \mid B_{h}$ is isotopic rel $\partial B_{h}$ to a \pm 4 -Dehn twist (Lemma 2.4 part $2(\mathrm{~d})$ ). If $k$ is a prime knot, then a contradiction follows from the proof of Claim 2 in Theorem 5.3. If $k$ is composite, then a contradiction follows from the proof of Claim 3 in Theorem 5.3. Hence, Case 1 is impossible.

Case 2. Some component of $V$ is a regular neighborhood of an annulus in $h_{K}-\left\{B_{h}\right\}$.

Then, by Lemma 5.21, part 2 such an annulus is adjacent to $B_{h}$. Let $A_{1}, \ldots, A_{p}$ be the annuli in $h_{K}$ adjacent to $B_{h}\left(p=\left|\partial B_{h}\right|\right)$ and let $S_{i}, 1 \leq$ $i \leq p$, be the components of $h_{K}-\left\{B_{h}\right\}$ adjacent to $A_{i}$. Lemma 5.21, part 1, then, says that $\varphi \mid S$ is admissibly isotopic to a periodic map where

$$
S=B_{h} \cup \bigcup_{i=1}^{p}\left(A_{i} \cup S_{i}\right) .
$$

Hence the adjoint automorphism of $S_{i}$ is the monodromy of a cable of $S^{1} \times D^{2}$ or a torus knot in $S^{3}$ of type $(r, s)$, say. From Lemma $2.4 \varphi^{p q r s} \mid S$ is isotopic to a product of $(q-p r s)$-Dehn twists along the cores of $A_{i}$ 's. Since $q-p r s \neq$ 0 for any $r, s$, the automorphism $\varphi \mid S$ is not isotopic to a periodic map, a contradiction. Theorem 5.4 is proved.

\section{8}

8.1. Iterated torus knots, cable knots. Throughout this subsection $(p, q)$ denotes the $(p, q)$ torus knot, $J_{(p, q)}$ the $(p, q)$ cable of $S^{1} \times D^{2}$, and $\sigma(\cdots)$ the signature of a knot in a homology sphere. When $S$ is a closed surface, the following lemma is the same as Lemma 4.2 of [2]. A similar proof applies to a bounded surface $S$, so we omit the proof.

Lemma 8.1. Let $g: S \rightarrow S$ be a periodic map. Suppose that $g$ extends over a nontrivial compression body $B$. Then there is an essential simple loop $C$ in $\partial_{+} B$ such that $C$ bounds a disk in $B$, and for each $k, f^{k}(C)=C$ or $f^{k}(C) \cap C=\varnothing$. In particular, if $g$ is irreducible, then $C$ is parallel to $\partial S$.

A knot $K \subset S^{3}$ is a graph knot if the knot exterior of $K$ is a graph manifold. Every graph knot is obtained by repeatedly taking a cable or connected sum starting from the unknot, and hence it is a fibered knot.

Theorem 8.2. Let $K_{1}, \ldots, K_{n}$ be prime graph knots such that $K_{1} \# \ldots \# K_{n} \geq 0$. Then there are a partition $\{1, \ldots, n\}=\coprod_{s=1}^{m}\left\{i_{s}, j_{s}\right\} \coprod_{t=1}^{l} \alpha_{t}$ (hence $n=2 m+l$ ) and graph knots $K_{i}^{\prime}, 1 \leq i \leq n$, satisfying the following properties.

1. $K_{i_{s}}^{\prime} \cong K_{j_{s}}^{\prime}$ for $1 \leq s \leq m$, and $K_{\alpha_{t}}^{\prime}=0$ for $1 \leq t \leq l$.

2. Each $K_{i}$ is ribbon concordant to $K_{i}^{\prime}$ by successive reductions of type $J(k \#-k) \geq J(0)$. In particular, $K_{i}^{\prime}$ is the unknot or a prime graph knot.

As immediate consequences we obtain

Corollary 8.3. A graph knot $K$ is homotopically ribbon if and only if we can obtain the unknot from $K$ by successive reductions of type $J(k \#-k) \geq J(0)$. 
Corollary 8.4. Let $K_{1}, \ldots, K_{n}$ be iterated torus knots such that $K_{1} \# \ldots \# K_{n} \geq$ 0 . Then $\{1, \ldots, n\}$ can be paired into $\coprod_{s=1}^{m}\left\{i_{s}, j_{s}\right\}$ (hence $n=2 m$ ) such that $K_{i_{s}} \cong-K_{j_{s}}$.

Remark. Corollary 8.4 shows that an iterated torus knot is not ribbon. In fact, it is not difficult to see that an iterated torus knot is not slice by showing that the Alexander polynomial is not of the form $f(t) f\left(t^{-1}\right)$.

Proof of Theorem 8.2. Let $K=K_{1} \# \ldots \# K_{n}$. Let the automorphism $\varphi$ of $H_{K}$ be an extension of the closed monodromy of $K$. Note that the adjoint automorphism of a nontrivial component of $h_{K}-\left\{B_{h}\right\}$ is the monodromy of a cable of a solid torus or a torus knot.

We prove the theorem by induction on $\left|h_{K}\right|$. If $\left|h_{K}\right|=3$, then $n=2$ and $K=K_{1} \# K_{2}$, a connected sum of torus knots. By Lemma 8.1 and the fact that a torus knot is not slice, each component of $h_{K}$ is incompressible in $H_{K}$, so that $K_{1} \cong-K_{2}$ by Theorem 5.3 or Proposition 6.1. Suppose Theorem 8.2 is true for all $K^{\prime}$ such that $\left|h_{K^{\prime}}\right|<\left|h_{K}\right|$.

Case 1. There is a nontrivial component of $h_{K}$ compressible in $H_{K}$.

Let $S \in h_{K}$ be a compressible nontrivial component such that $\partial_{r} S>\partial_{r} R$ for every such $R \in h_{K}$. Without loss of generality $S$ is contained in the fiber of $K_{1}$. Let $m$ be the least positive integer such that $\varphi^{m}(S)=S$. Let $J_{1}$ be the fibered knot with monodromy $\varphi^{m} \mid S$; then $J_{1}$ is a nontrivial cable of $S^{1} \times D^{2}$ or a nontrivial torus knot. Note that either $K_{1}=J_{0}\left(J_{1}(L)\right)$ or $K_{1}=J_{0}\left(J_{1}\right)$ for some $J_{0}\left(\subset S^{1} \times D^{2}\right)$ and nontrivial graph knot $L$. Take a maximal compression body $B$ for $S$ in $H_{K}$; after an isotopy $\varphi^{m}(B)=B$. Let $D^{\prime}$ be a component of $\overline{\partial B-\partial_{+} B}$ such that $\partial D^{\prime}$ is a component of $\partial S-\partial_{r} S$. Note that $D^{\prime}=\varnothing$ if $J_{1}$ is a torus knot.

Subcase 1. $D^{\prime}$ is not a disk.

We show this is absurd. Let $D$ be the component of $\overline{\partial B-\partial_{+} B}$ such that $\partial D=\partial_{r} S$. Since $D^{\prime}$ is not a disk, by Lemma $8.1 D$ is a disk. Let $S^{\prime}=$ $S \cup_{\partial_{r} S} D$. By Lemma $2.5 \varphi^{m} \mid S^{\prime}$ is irreducible. Regard $B$ as a compression body of $S^{\prime}$. Then, by Lemma $8.1 B$ is a trivial compression body of $S^{\prime}$, so that $S^{\prime}$ is incompressible in $H$. Hence $S^{\prime}$ is not a closed surface, implying $D^{\prime} \cong S^{\prime}$. Since $D^{\prime} \neq \varnothing, J_{1}$ is not a torus knot. Let $H^{\prime}$ be the component of $\overline{H-B}$ containing $D^{\prime}$. The automorphism $\varphi^{m} \mid \partial H^{\prime}$ is the closed monodromy of $J_{1}(L)$, hence $k=J_{1}(L) \geq 0$. Since each nontrivial component of $h_{k}$ is incompressible in $H^{\prime}$, applying Theorem 5.4 to $k$ shows that $J_{1}$ is a trivial cable, a contradiction.

Subcase 2. $D^{\prime}$ is a disk.

Then $J_{1}$ is a nontrivial cable of $S^{1} \times D^{2}$, and $K_{1}$ has a presentation $J_{0}\left(J_{1}(L)\right)$. Let $T$ be the nonannulus component of $h_{K}$ such that $\partial_{r} T$ is parallel to $\partial D^{\prime}$ in $\partial H$; then $T$ is a trivial component by the maximality of $\partial_{r} S$. Let $l$ be the least positive integer such that $\varphi^{l}(T)=T$, and $H^{\prime \prime}$ be the closure of the component of $H-D^{\prime}$ containing $T$. Then $L$ is the composite graph knot with closed monodromy $\varphi^{l} \mid \partial H^{\prime \prime}$, so that $L \geq 0$. Since $\left|h_{L}\right|<\left|h_{K}\right|$, the induction hypothesis implies $L$ is ribbon concordant to the unknot by successive reductions of type $J(k \#-k) \geq J(0)$. Hence $K_{1}$ is ribbon concordant to $K_{1}^{\prime}=J_{0}\left(J_{1}(0)\right)$ by reductions of this type, and we have $K^{\prime}=K_{1}^{\prime} \# K_{2} \# \cdots \# K_{n} \geq 0$. Since $\left|h_{K^{\prime}}\right|<\left|h_{K}\right|$, Theorem 8.2 holds for $K^{\prime}$. Note that if $J_{0}, J_{1}$ are a $(p, 1)$ cable and a $(q, 1)$ cable for some $p, q$, then 
$K_{1}^{\prime}=0$; otherwise, $K_{1}^{\prime}$ is a prime graph knot. Hence Theorem 8.2 also holds for $K$.

Case 2. Every nontrivial component of $h_{K}$ is incompressible in $H_{K}$.

First we show that $(*)$ there are no $S_{0}, S_{1} \in h_{K}-\left\{\widehat{D}_{n}\right\}$ such that $S_{0}, S_{1}$ are adjacent via an annulus and $S_{1}=S_{0}^{*}$. If there is a nonannulus component $S \in h_{K}-\left\{B_{h}\right\}$ such that the fibered knot with monodromy $f_{S}$ has a dual knot, then $f_{S}$ leaves some component of $\partial S-\partial_{r} S$ invariant. Then, $S$ is a trivial component. Since two trivial components cannot be adjacent via an annulus, we obtain $(*)$.

If $n=1, K_{1}$ cannot be a nontrivial cable knot (Theorem 5.4), a contradiction. So suppose $n \geq 2$. From the proof of Lemma 5.10 there are a partition $\mathscr{P}$ of $\{1, \ldots, n\}$ and prime graph knots $K_{i}^{\prime}, 1 \leq i \leq n$, such that $K_{i} \geq K_{i}^{\prime}$, and for each $P \in \mathscr{P}, K^{\prime}=K_{P}^{\prime}=\#_{i \in P} K_{i}^{\prime} \geq 0$, and $h_{K^{\prime}}$ is useful for $H_{K^{\prime}}$. As in Subcase 2 of Case 1 above the induction hypothesis implies $K_{i}$ is ribbon concordant to $K_{i}^{\prime}$ by successive reductions of type $J(k \#-k) \geq J(0)$. Obviously $h_{K^{\prime}}$ also satisfies the condition $(*)$ above, hence $U_{K^{\prime}}$ does not contain any annulus components of $h_{K^{\prime}}$ by Lemma 5.19, part 1. Since $\left|h_{K}\right| \geq\left|h_{K^{\prime}}\right|$, we may assume $|\mathscr{P}|=1$ and $K_{i}=K_{i}^{\prime}$. It follows from Proposition 5.23 that $n=2$. By Proposition 5.1 there are $\widetilde{K}_{1}, \widetilde{K}_{2}$ such that $\widetilde{K}_{1} \cong-\widetilde{K}_{2}$ and $\widetilde{K}_{i}$ is obtained from $K_{i}$ by successive reductions of type $J(k \#-k) \geq J(0)$. Thus $K_{i}$ is ribbon concordant to $\widetilde{K}_{i}$, and $\widetilde{K}_{i}$ is the unknot or a prime graph knot. This completes the proof of Theorem 8.2.

Example 1. The connected sum of four iterated torus knots below is algebraically slice but not homotopically ribbon.

$$
(2,13 ; 2,3) \#(2,15) \#(2,-15 ; 2,-3) \#(2,-13)
$$

where $\left(p_{1}, q_{1} ; p_{2}, q_{2}\right)$ denotes the $\left(p_{1}, q_{1}\right)$ cable knot about the $\left(p_{2}, q_{2}\right)$ torus knot. (This iterated torus knot is denoted by $\left(q_{1}, p_{1} ; q_{2}, p_{2}\right)$ in [22].) Livingston and Melvin [22] prove that the knot is algebraically slice. The fact that it is nonribbon is an immediate consequence of Corollary 8.4.

Any $(p, 1)$ cable of a (homotopically) ribbon fibered knot is (homotopically) ribbon. The following theorem hence shows that all homotopically ribbon, cable fibered knots in homotopy spheres are such knots if Thurston's geometrization conjecture is true.

Theorem 8.5. Let $J_{(p, q)}(k)$ be a $(p, q)$ cable of a nontrivial fibered knot $k$ in a homology sphere $M$ where $p>1$. If $n J_{(p, q)}(k) \geq 0$ for some $n>0$, then the following hold.

1. $J_{(p, q)}(k) \geq 0$.

2. $q= \pm 1$; if $p>2$, then $k \geq 0$.

3. If $M$ is a homotopy sphere and 3-manifold groups are residually finite, then $k \geq 0$ and $q= \pm 1$.

Proof. If $k \geq 0$, then the assumption $n J_{(p, q)}(k) \geq 0$ implies that $n(p, q)$ is algebraically slice, hence $\sigma((p, q))=0$. It follows that $q= \pm 1$ and $J_{(p, q)}(k) \geq$ $(p, \pm 1)=0$. Therefore, we may assume $k \nsupseteq 0$. 
Proof of part 1. Let $n$ be the least positive integer such that $n J_{(p, q}(k) \geq 0$. Assume for a contradiction that $n>1$. Let $J_{i}=J_{(p, q)}, 1 \leq i \leq n$, with fiber $F_{i}$. Let $k_{i}=k, K_{i}=J_{i}\left(k_{i}\right)$ for $1 \leq i \leq n$; then $K_{1} \# \ldots \# K_{n}=K \geq 0$. The closed monodromy of $K$ extends to an automorphism $\varphi$ of a handlebody $H_{K}$. If $n>2$, let $A_{i}, 1 \leq i \leq n$, be the annuli in $h_{K}$ adjacent to $\widehat{D}_{n}$. Otherwise, let $A_{i}=\varnothing$. We may regard $F_{i}$ as the component of $h_{K}-\left\{\widehat{D}_{n}\right\}$ adjacent to $A_{i}$. Let $B_{j}, 1 \leq j \leq n p$, be the annuli in $h_{K}-\left\{\bigcup A_{i}\right\}$ adjacent to $F_{i}, 1 \leq i \leq n$, such that $B_{i} \cap F_{i} \neq \varnothing$ for $1 \leq i \leq n$. Let $S$ be the union of the $n p$ components of $\overline{\partial H_{K}-\bigcup_{j=1}^{n p} B_{j}}$ which are disjoint from $\widehat{D}_{n}$. Take a maximal compression body $B$ for $S$ in $H_{K}$, and set $H^{\prime}=\overline{H_{K}-B}$, a handlebody. Since a maximal compression body is unique up to isotopy, we may assume $\varphi\left(H^{\prime}\right)=H^{\prime}$.

For $1 \leq i \leq n$ the compression body $B$ induces a homotopically ribbon concordance $k_{i} \geq k_{i}^{\prime}$ where $k_{i}^{\prime}$ is a nontrivial fibered knot in a homology sphere. Note that the fibered knots $k_{i}^{\prime}, 1 \leq i \leq n$, are not necessarily of the same knot type. The $\varphi \mid \partial H^{\prime}$ is the closed monodromy of $K^{\prime}=J_{1}\left(k_{1}^{\prime}\right) \# \cdots \# J_{n}\left(k_{n}^{\prime}\right)(\geq 0)$.

Let us define a boundary pattern $h^{\prime}$ of $H^{\prime}$. Take a 1-submanifold $C$ of $\operatorname{Fr} B$ with the minimal number of components such that $\varphi(C)=C$, and $\varphi \mid(\operatorname{Fr} B-C)$ is a union of irreducible automorphisms and periodic ones up to isotopy. Define

$$
h^{\prime}=\left\{\text { the components of } h_{K} \cap H^{\prime}, N(C) \text {, and } \overline{\operatorname{Fr} B-N(C)}\right\} .
$$

Since the ambient manifold of $J_{i}\left(k_{i}^{\prime}\right)$ is not necessarily a homotopy sphere, $\varphi^{p} \mid F_{i} \cup B_{i} \cup S$ is possibly isotopic to a periodic map, where $S\left(\neq F_{i}\right)$ is a component of $h^{\prime}$ adjacent to $B_{i}$. That is, $h^{\prime}$ is not necessarily equal to $h_{K^{\prime}}$.

Claim. $h^{\prime}$ is a useful boundary pattern for $H^{\prime}$.

Proof. By the maximality of $B, \operatorname{Fr} B$ is incompressible in $H^{\prime}$. Hence each component of $h^{\prime} \cap \operatorname{Fr} B$ is incompressible. By the minimality of $n, \widehat{D}_{n}$ is incompressible in $H^{\prime}$. Since $\varphi \mid F_{i}$ is isotopic to an irreducible periodic map, if $F_{i}$ is compressible, so is $\partial F_{i}$ in $H^{\prime}$ (Lemma 8.1). This contradicts the incompressibility of $\widehat{D}_{n}$ or $\operatorname{Fr} B$. Therefore each component of $h^{\prime}$ is incompressible in $H^{\prime}$, so that $h^{\prime}$ is a useful boundary pattern.

Thus $\left(H^{\prime}, h^{\prime}\right)$ has a characteristic submanifold $X$. Define the subsets $U$ and $V$ of $X$ as in $\S 5.1$. It is easily verified that Lemmas 5.11-5.17 hold for these $U$ and $V$. For any component of $h^{\prime}$ the adjoint automorphism is irreducible or isotopic to a periodic map (cf. part 3 of Proposition 5.1). Thus Lemma 5.21, part 1 holds for $V$.

Let us deduce a contradiction by assuming $n>2$. Since $\varphi \mid F_{i} \not$ id, each $A_{i}$ is not contained in $U$ but in $V$. By Lemma 5.21, part 1 we also see that no component of $\operatorname{Fr} V$ is parallel to $A_{i}$. Let $\alpha_{i}$ be the component of $\operatorname{Fr} V$ associated to the simple loop $\partial A_{i} \cap \partial \widehat{D}_{n}$. Since $\partial_{0} \alpha_{i}$ is invariant under $\varphi$, so is $\partial_{1} \alpha_{i}$. No components of $\partial F_{i}-\partial_{r} F_{i}$ are invariant under $\varphi$, so that $\partial_{1} \alpha_{i} \subset \partial H^{\prime}-\operatorname{Fr} B$. It follows that $\partial_{1} \alpha_{i} \subset \widehat{D}_{n}$ or $\partial_{1} \alpha_{i} \subset F_{j}(i \neq j)$ (and $\partial_{1} \alpha_{i}$ is parallel to $\partial_{r} F_{j}$ ). In either case we get a contradiction by constructing a 3-manifold violating Lemma 5.27. (If $\partial_{1} \alpha_{i_{0}} \subset F_{j}$ for some $i_{0} \neq j$, follow the proof of Lemma 5.26, part 2. If $\partial_{1} \alpha_{i} \subset \widehat{D}_{n}$ for all $i$, the closure of the outermost component of $H^{\prime}-\bigcup \alpha_{i}$ contradicts Lemma 5.27.) Thus it follows that $n=2$.

Corollary 3.2 of [25] shows that $\left(S^{1} \times D^{2}, J_{1}\right) \not\left(-S^{1} \times D^{2},-J_{1}\right)$, hence 
$\widehat{D}_{2} \not \subset U$, i.e., $\widehat{D}_{2} \subset V$. Again, using the fact that no component of $\partial F_{i}-$ $\partial_{r} F_{i}$ are invariant under $\varphi$, we see that some component of $V$ is a regular neighborhood of $\widehat{D}_{2}$. By Lemma 5.21, part $1 \varphi \mid F_{1} \cup \widehat{D}_{2} \cup F_{2}$ is then isotopic to a periodic map. However, on this set $\varphi^{p q}$ is isotopic to a \pm 2 -Dehn twist along the core of $\widehat{D}_{2}$, which is not isotopic to a periodic automorphism of $F_{1} \cup \widehat{D}_{2} \cup F_{2}$. This is absurd, completing the proof of part 1 .

Proof of parts 2 and 3 . Recall that if $k \geq 0$, then the stated results follow. We shall deduce a contradiction by assuming $k \nsupseteq 0$. We proceed similarly to the proof of part 1. As usual let $\varphi$ denote an automorphism of a handlebody $H_{K}$, an extension of the closed monodromy of $K$. Set $F^{\prime}=B_{h_{K}}$, a union of the fiber of the $(p, q)$ cable of $S^{1} \times D^{2}$ and a disk. Let $A_{1}, \ldots, A_{p}$ be the annuli components of $h_{K}$ adjacent to $F^{\prime}$, and set $S=\overline{\partial H_{K}-F^{\prime} \cup \bigcup_{i=1}^{p} A_{i}}$. Then $S$ has $p$ components and $\varphi^{p}$ restricts to the monodromy of $k$ on each component of $S$. Take a maximal compression body $B$ for $S$ in $H_{K}$ and set $H^{\prime}=\overline{H-B}$. Without loss of generality $\varphi\left(H^{\prime}\right)=H^{\prime}$. Let $k^{\prime} \not 00$ be the fibered knot in a homology sphere whose monodromy is the restriction of $\varphi$ to a component of $\operatorname{Fr} B$. Then $k \geq k^{\prime}$ and $\varphi \mid \partial H^{\prime}$ is the closed monodromy of $K^{\prime}=J_{(p, q)}\left(k^{\prime}\right)(\geq 0)$. Let $C$ be a 1-submanifold of $\operatorname{Fr} B$ defined as in the proof of part 1. Define

$$
h^{\prime}=\left\{F^{\prime}, A_{1}, \ldots, A_{p}, \text { the components of } \overline{\operatorname{Fr} B-N(C)}\right\} .
$$

As in part $1 h^{\prime}$ is a useful boundary pattern of $H^{\prime}$. Here we first prove part 3. If $k$ is a knot in a homotopy sphere and 3-manifold groups are residually finite, then $k^{\prime}$ and hence $K^{\prime}$ are knots in homotopy spheres (Lemma 1.2). By Swarup [24, Lemma 2.1] $h^{\prime}$ equals $h_{K^{\prime}}$. Hence we can apply Theorem 5.4 to $K^{\prime}$, so that $p=1$, a contradiction. This establishes the proof of part 3 .

Let us prove part 2. When $p=2$, by [23, Theorem 9] or [14, Theorem 12] $\sigma((2, q))=\sigma\left(J_{(2, q)}(k)\right)$, which is equal to 0 . Hence, we obtain the desired result $q= \pm 1$. We may assume $p>2$. As in $\$ 5.1$ define $U, V$ for a characteristic submanifold $X$ of $\left(H^{\prime}, h^{\prime}\right)$.

Claim 1. $U$ contains no annulus components of $h^{\prime}$.

Proof. Suppose not. Let $U_{0}$ be a component of $U$ containing an annulus component of $h^{\prime}$. First suppose that $U_{0}$ contains some $A_{i}$. Since $\varphi \mid F^{\prime}$ is irreducible, $U_{0} \cap F^{\prime}$, a lid of $U_{0}$, is isotopic to $F^{\prime}$. In particular, $U_{0} \cap F^{\prime}$ is invariant under $\varphi$. However, the other lid of $U_{0}, L$ say, is contained in some components of $\operatorname{Fr} B$. The automorphism $\varphi$ permutes the components of $\operatorname{Fr} B$ cyclically, hence $L$ is not invariant under $\varphi$, a contradiction. Thus $U_{0}$ contains an annulus in $h^{\prime} \cap \operatorname{Fr} B$. Then $\partial U_{0} \cap \operatorname{Fr} B$ consists of the two lids of $U_{0}$ and (a nonempty set of) annuli connecting them, so that $\operatorname{Fr} B$ is compressible in $H^{\prime}$. This is a contradiction.

Claim 2. If $q \neq \pm 1$, no component of $\operatorname{Fr} V$ is parallel to an annulus in $h^{\prime}$.

Proof. Assume for a contradiction that a component of $\operatorname{Fr} V$ is parallel to some $A_{i}$. Then, there are the components $V_{1}, \ldots, V_{p}$ of $V$ such that $V_{i}$ is a regular neighborhood of $A_{i}$. Let $M$ be the components of $\overline{H^{\prime}-X}$ containing $\bigcup_{i=1}^{p} \operatorname{Fr} V_{i}$. Then $M$ is a simple manifold with a certain boundary pattern, in particular the automorphism $\varphi \mid M$ is admissibly isotopic to a periodic map. The boundary pattern of $M$ induced from $h^{\prime}$ consists of $\overline{F^{\prime}-\bigcup_{i=1}^{p} V_{i}}, \operatorname{Fr} V_{1}, \ldots$, Fr $V_{p}$, submanifolds $T_{1}$ of $\operatorname{Fr} B$, and properly embedded annuli $T_{2}$ such that 
$\partial T_{2} \subset \operatorname{Fr} B$. Since $\varphi$ permutes the components of Fr $B$ cyclically, the automorphism $\varphi \mid T_{1}$ cannot be isotoped to have fixed points. The automorphism $\varphi \mid T_{2}$ is not isotopic to a periodic map with fixed points; otherwise, $\varphi$ acts on some (annulus) component of $T_{2}$ as involution, contradicting $|\operatorname{Fr} B|=p>2$. On the other hand, since $q \neq \pm 1, \varphi \mid F^{\prime}$ is isotopic to a periodic map with just one fixed point. Therefore, the fixed point datum of $\varphi \mid \partial M$ cannot cancel. It follows that $\varphi \mid \partial M$ is not null-cobordant, a contradiction.

Now Claims 1 and 2 contradict Lemma 5.22, hence we have $q= \pm 1$. Claim 3 below shows that Claim 2 is true even if $q= \pm 1$. We again get a contradiction by Lemma 5.22, completing the proof of Theorem 8.5.

Claim 3. No component of $\operatorname{Fr} V$ is parallel to an annulus in $h^{\prime}$.

Proof. We may assume $q= \pm 1$. Thus $F^{\prime}$ is a sphere with $p$ punctures and $\varphi$ permutes the components of $\partial F^{\prime}$ cyclically. Let $S_{i}, 1 \leq i \leq p$, be the component of $h^{\prime}-\left\{F^{\prime}\right\}$ which is adjacent to $A_{i}$, and set $S=F^{\prime} \cup \bigcup_{i=1}^{p}\left(A_{i} \cup S_{i}\right)$. For a contradiction assume Claim 3 is false. Isotop $\varphi$ admissibly so that $\varphi \mid S$ is a periodic map (Lemma 5.21). Then $\varphi \mid S$ is of period $p$ and has two fixed points of type $1 / p$. Since $\varphi^{p} \mid S_{i}=\mathrm{id}, S_{i}$ and hence $S$ are punctured disks. (Otherwise, the knot exterior of $k^{\prime}$ contains a nonseparating torus.) This shows that the closed monodromy of $k^{\prime}$ is that of a connected sum of certain $m$ prime fibered knots where $m=\left|\partial S_{i}\right|-1$. Remove $F^{\prime}, A_{i}$ and $S_{i}, 1 \leq i \leq p$, from $h^{\prime}$ and add $S$. We then have the new boundary pattern $\tilde{h}$ for $H^{\prime}$. If $S$ is incompressible in $H^{\prime}, \tilde{h}$ is useful. Define $U^{\prime}$ and $V^{\prime}$ for a characteristic submanifold of $\left(H^{\prime}, \tilde{h}\right)$. We can easily verify Claims 1 and 3 hold for $U^{\prime}, V^{\prime}$. Lemma 5.22 then gives a contradiction.

Suppose $S$ is compressible in $H^{\prime}$. By Lemma 8.1 a maximal compression body of $S$ in $H^{\prime}$ can be obtained by compressing a set of pairwise disjoint, essential simple loops which is invariant under the action of $\varphi$. Let $\left\{l_{j}\right\}_{j}$ be such a set such that $l_{j}$ bound pairwise disjoint disks $D_{j}$ in $H^{\prime}$ invariant under $\varphi$. Let us consider $\bar{l}_{j}=q\left(l_{j}\right)$ in $\bar{S}=S / \varphi$ where $q: S \rightarrow \bar{S}$ is the natural projection. Note that $\bar{S}$ is a disk with $m$ punctures having two cone points. Suppose that some $\bar{l}_{j}$ bounds a disk in $\bar{S}$ containing the two cone points of $\bar{S}$. Then $S-\bigcup_{k=1}^{p} \varphi^{k}\left(l_{j}\right)$ consists of a disk with $p$ punctures and $p$ copies of a disk with $m$ punctures, say, $S_{1}^{\prime}, \ldots, S_{p}^{\prime}$. Let $H_{1}$ be the closure of a component of $H^{\prime}-\bigcup_{k=1}^{p} \varphi^{k}\left(D_{j}\right)$ containing $S_{1}^{\prime}$; then $\varphi^{p}\left(H_{1}\right)=H_{1}$. Since $\varphi^{p} \mid S_{1}^{\prime}=\mathrm{id}$, we see that $\varphi^{p} \mid \partial H_{1}$ is the closed monodromy of $k^{\prime}$. It follows $k \geq$ $k^{\prime} \geq 0$, a contradiction. Thus there is a component $C$ of $\bar{S}-\bigcup \bar{l}_{j}$ containing a component of $\partial \bar{S}$ and a cone point. Define the handlebody $H^{\prime \prime}$ to be the closure of the component of $H^{\prime}-\bigcup_{j} D_{j}$ containing the punctured disk $q^{-1}(C)$; then $\varphi\left(H^{\prime \prime}\right)=H^{\prime \prime}$. Let $s^{\prime}$ be the union of $q^{-1}(C)$ and the disks $D_{j}$ contained in $\partial H^{\prime \prime}$, so that $\left|\partial S^{\prime}\right|(\neq 0)$ is a multiple of $p$. Since $S^{\prime}$ is incompressible in $H^{\prime \prime}$, the boundary pattern $h^{\prime \prime}=\left(\overline{\partial H^{\prime \prime}-S^{\prime}} \cap h^{\prime}\right) \cup\left\{S^{\prime}\right\}$ for $H^{\prime \prime}$ is useful. Just like $\varphi|S, \varphi| S^{\prime}$ is of period $p$. Hence, defining $U^{\prime}, V^{\prime}$ for a characteristic submanifold of $\left(H^{\prime \prime}, h^{\prime \prime}\right)$, we see that Claims 1 and 3 hold for these $U^{\prime}$ and $V^{\prime}$. This gives a contradiction as above, completing the proof of Claim 3.

Example 2. Let $k$ be a -amphicheiral fibered knot in a homotopy sphere with an irreducible Alexander polynomial $\Delta(t)$. Let $K_{m}=J_{(m, 1)}(k)$. Then for any $n>0, n K_{2 m}, 2 n K_{2 m+1}$ are algebraically slice but not homotopically ribbon. 
Remark. The proof of algebraically-sliceness is a slight generalization of the proof of Example 4.2 in Kawauchi [20]. The arguments show that $K_{2 m}$ is algebraically slice if a knot $k$ has an irreducible Alexander polynomial satisfying the condition $(*)$ below.

Proof. (1) Algebraically-sliceness. Since $k$ is -amphicheiral, by [17] $\Delta(t)$ satisfies

$$
\Delta\left(t^{2}\right)=f(t) f(-t), f\left(t^{-1}\right) \doteq f(-t) \text { for some } f(t) \in Z[t] .
$$

It is well known that the Alexander polynomial of $K_{m}$ is $\Delta\left(t^{m}\right)$. We claim that $f\left(t^{m}\right)$ and $f\left(-t^{m}\right)$ are relatively prime in $Z[t]$. It suffices to show that $f(t)$ and $f(-t)$ are relatively prime in $Z[t]$. Let $g(t) \in Z[t]$ be a g.c.d. of $f(t)$ and $f(-t)$. Then $h(t)=g(t) g(-t)$ divides $f(t) f(-t)$ and hence $\Delta\left(t^{2}\right)$. Since $h(t)$ is an even function, $\Delta(t)$ has a factor $h\left(t^{1 / 2}\right) \in Z[t]$. Without loss of generality we may assume that $\operatorname{deg} g=\operatorname{deg} f$; then $f(t)= \pm f(-t)$. It follows that $t^{1 / 2} f\left(t^{1 / 2}\right)$ or $f\left(t^{1 / 2}\right)$ is a $Z$-polynomial, contradicting the irreducibility of $\Delta(t)$. Thus $f\left(t^{m}\right)$ and $f\left(-t^{m}\right)$ are relatively prime, so that [20, Lemma 4.1(3)] shows that $K_{2 m}$ is algebraically slice.

Let us show that $2 K_{2 m+1}$ is algebraically slice. By [20, Proposition 4.1(3)] $2 K_{2 m+1}$ is algebraically cobordant to $J_{(2 m+1,1)}(2 k)$. Since $2 k \cong k \#-k$ is slice, $2 K_{2 m+1}$ is algebraically slice.

(2) If $n K_{m} \geq 0$ for some $n>0$, then $K=K_{m} \geq 0$ by Theorem 8.5, part 1. As in the proof of Theorem $5.5(\S 5.1)$ the irreducibility of $\Delta(t)$ implies that $\partial H_{K}-B_{h}$ is incompressible in $H_{K}$. An application of Theorem 5.4 to $K$ gives a contradiction.

Theorem 8.5, parts 2 and 3 lead us to the question: if the $(2,1)$ cable of a knot $k$ is homotopically ribbon, is $k \geq 0$ ? The example below shows that the answer is "no" for a $(2,1)$ cable knot in a homology sphere. Hence, Theorem 5.4 and Example 2 cannot be generalized to a fibered knot in a homology sphere.

Example 3. Let $N$ be the homology sphere obtained by a $-\frac{1}{2}$-Dehn surgery of $S^{3}$ along the figure eight knot $k$. Let $k^{\prime}$ denote the core of the glued solid torus. Then $J_{(2,1)}\left(k^{\prime}\right)$ is a homotopically ribbon fibered knot in $N$.

Proof. Let $\varphi: F \rightarrow F$ be the closed monodromy of $K=J_{(2,1)}(k)$. Let $F_{1}, F_{2}$ be the two components of $\overline{F-B_{h_{K}}}$. Then $\varphi^{2} \mid F_{1}: F_{1} \rightarrow F_{1}$ is the monodromy of $k$ and $\varphi^{2} \mid B_{h}$ is a -4 -Dehn twist.

Claim. There is a diffeomorphism $G: F_{1} \rightarrow-F_{1}$ such that $G^{2}=\varphi^{2} \mid F_{1}$.

Proof of claim. Let $x, y$ be simple loops on $F_{1}$ intersecting in a single point, and $\tau_{1}, \tau_{2}$ denote the Dehn twists along $x, y$ respectively. Without loss of generality the monodromy $\varphi^{2} \mid F_{1}=\tau_{1} \circ \tau_{2}^{-1}$. Hence, letting $r$ be an orientation reversing diffeomorphism of $F_{1}$ exchanging $x$ and $y$, we obtain $\left(r \circ \tau_{2}^{-1}\right)^{2}=\tau_{1} \circ \tau_{2}^{-1}$. Hence, $r \circ \tau_{2}^{-1}$ is the claimed diffeomorphism.

Denote by $\tau$ a Dehn twist along a simple loop on $F_{1}$ parallel to $\partial F_{1}$. Then $\tau^{2} \circ\left(\varphi^{2} \mid F_{1}\right)$ is the monodromy of $k^{\prime}$ in $N$, and $\tau^{2} \circ \varphi$ is the closed monodromy of $J_{(2,1)}\left(k^{\prime}\right)$. Let $\psi=\tau^{2} \circ \varphi$. Identify $F_{1} \cup F_{2}$ and $F_{1} \times\{0,1\}$ by id: $F_{1} \rightarrow$ $F_{1} \times 0$ and $G \circ\left(\varphi^{-1} \mid F_{2}\right): F_{2} \rightarrow F_{1} \times 1$. Under this identification we have

$$
\begin{aligned}
& \psi(x, 1)=\left(\tau^{2} \circ \varphi^{2} \circ G^{-1}(x), 0\right), \\
& \psi(x, 0)=(G(x), 1), \quad \text { where } x \in F_{1} .
\end{aligned}
$$


We can identify $B_{h_{K}}$ and $\partial F_{1} \times I$ so that

$$
\psi \mid \partial F_{1} \times I=\eta^{-2} \circ r,
$$

where $\eta$ is a Dehn twist along the core of $\partial F_{1} \times I$, and $r$ is an involution of $\partial F_{1} \times I$ such that $r(x, t)=(G(x), 1-t)$. Since $\varphi^{2} \mid F_{1}=G^{2}$ and $\tau^{2} \circ \eta^{-2} \simeq$ id in $\partial\left(F_{1} \times I\right)$, after an isotopy we obtain

$$
\psi(x, t)=(G(x), 1-t) \quad \text { in } \partial\left(F_{1} \times I\right) .
$$

It is easy to see $\psi$ extends over the handlebody $F_{1} \times I$, hence $J_{(2,1)}\left(k^{\prime}\right) \geq 0$.

Remark. As pointed out in the proof the monodromies of $J_{(2,1)}(k)\left(\subset S^{3}\right)$ and $J_{(2,1)}\left(k^{\prime}\right)(\subset N)$ differ only by a Dehn twist along a null-homologous loop. Therefore the two monodromies induce equivalent isomorphisms on the homology groups.

8.2. A class $\mathscr{K}$. Define $\mathscr{K}$ to be a class of prime fibered knots in homotopy spheres consisting of $J_{1}\left(\cdots\left(J_{m}(k)\right) \cdots\right)$ and $k$ where $J_{i}$ and $k$ satisfy the following conditions.

1. $k$ is a torus knot or a prime fibered knot in homotopy spheres such that no nontrivial Laurent polynomial $f(t) f\left(t^{-1}\right)$ divides $\Delta_{k}(t)$.

2. Each $J_{i}$ is a nontrivial cable of $S^{1} \times D^{2}$.

Theorem 8.6. If $K_{1}, \ldots K_{n} \in \mathscr{K}$ and $K_{1} \# \ldots \# K_{n} \geq 0$, then $\{1, \ldots, n\}$ can be paired into $\amalg_{s=1}^{m}\left\{i_{s}, j_{s}\right\}$ such that $K_{i_{s}} \cong-K_{j_{s}}$.

Proof of Theorem 8.6. First we consider the case $n>1$. Set $K=K_{1} \# \cdots \# K_{n}$. Let an automorphism $\varphi$ of $H_{K}$ be an extension of the closed monodromy of $K$. For each $1 \leq i \leq n$ let $K_{i}=J_{1}^{i}\left(\cdots\left(J_{m(i)}^{i}\left(k_{i}\right)\right) \cdots\right)$ be a satellite presentation satisfying the definition of $\mathscr{K}$. Let $S_{j}^{i}, S^{i}$ denote the fiber of $J_{j}^{i}, k_{i}$ respectively. Let $\mathscr{T}$ be a minimal set of tori in the complement of $K$ which decompose it into a Seifert fibered manifold and a simple manifold. Isotoping $\mathscr{T}$ so that $|F \cap(\bigcup \mathscr{T})|$ is minimal where $F$ is the fiber of $K$, and set $C=F \cap(\bigcup \mathscr{T})$. Note that each nonannulus component of $h_{K}-\left\{\widehat{D}_{n}\right\}$ is a copy of $S_{j}^{i}$ or contained in a copy of $S^{i}$ for some $i, j$.

Case 1. Every nontrivial component of $h_{K}$ is incompressible in $H_{K}$.

First note that condition 1 in the definition of $\mathscr{K}$ implies that $k_{i}$ is minimal with respect to " $\leq$ " among all fibered knots. If $K_{i}$ has presentation $J(k \#-k)$ or $\left.J_{1}\left(J^{*}(k)\right)\right)$ where $k \neq 0$ and $J \not\left(S^{1} \times D^{2}, S^{1} \times 0\right)$, then so does $k_{i}$. This implies $k_{i}$ is not minimal, contradicting the observation above. Then, Theorem 8.6 follows from Theorem 5.4, part 2 .

Case 2. There is a nontrivial component of $h_{K}$ compressible in $H_{K}$.

We shall deduce a contradiction in this case. As in the proof of Theorem 8.2 take a nontrivial compressible component $R \in h_{K}$ such that $\partial_{r} R>\partial_{r} R^{\prime}$ for every such a component $R^{\prime} \in h_{K}$. The assumption on $k_{i}$ implies that the fiber $S^{i}$ of $k_{i}$ is incompressible in $H_{K}$. Hence, $R$ is (a copy) of $S_{j_{0}}^{i_{0}}$ for some $i_{0}, j_{0}$, so that there is a compression disk $D$ in $H_{K}$ such that $\partial_{r} R=\partial D$ (this is because the monodromy of a cable of $S^{1} \times D^{2}$ is irreducible). Let $H^{\prime}$ be the closure of the component of $H_{K}-D$ containing $R$; then $\varphi^{m}\left(H^{\prime}\right)=H^{\prime}$. Set $\psi=\varphi^{m} \mid H^{\prime}$. Then, the closed monodromy of $L=J_{j_{0}}^{i_{0}}\left(J_{j_{0}+1}^{i_{0}}\left(\cdots\left(k_{i_{0}}\right) \cdots\right)\right)$ 
is $\psi \mid \partial H^{\prime}$, which extends over the handlebody $H^{\prime}\left(=H_{L}\right)$. Since $L$ is a nontrivial cable knot and $h_{L}$ is a useful boundary pattern for $H^{\prime}$, this contradicts Theorem 5.4.

To complete the proof of Theorem 8.6 we need to consider the case $n=1$. However, just as in the arguments in Case 2 above we can deduce a contradiction.

\section{REFERENCES}

1. I. Aitchison and D. Silver, On certain fibered ribbon disc pairs, Trans. Amer. Math. Soc. 306 (1988), 529-551.

2. F. Bonahon, Cobordism of automorphisms of surfaces, Ann. Sci. École Norm. Sup. 10 (1983), 237-270.

3. __ Ribbon fibered knots, cobordism of surface diffeomorphisms, and pseudo-Anosov diffeomorphisms, Math. Proc. Cambridge Philos. Soc. 94 (1983), 235-251.

4. A. Casson and C. Gordon, A loop theorem for duality spaces and fibered ribbon knots, Invent. Math. 74 (1983), 119-137.

5. A. Casson and D. Long, Algorithmic compression of surface automorphisms, Invent. Math. 81 (1985), 295-303.

6. M. Culler, C. Gordon, J. Luecke, and P. Shalen, Dehn surgery on knots, Ann. of Math. (2) 125 (1988), 237-300.

7. A. Edmonds and J. Ewing, Remarks on the cobordism group of surface diffeomorphisms, Math. Ann. 259 (1982), 497-504.

8. D. Eisenbud and W. Neumann, Three-dimensional link theory and invariants of plane curve singularities, Ann. of Math. Stud., no. 110, Princeton Univ. Press, Princeton, N.J., 1985.

9. M. Gerstenhaber and O. Rothaus, The solution of sets of equation in groups, Proc. Nat. Acad. Sci. U.S.A. 48 (1962), 1531-1533.

10. P. Gilmer, Ribbon concordance and a partial order on S-equivalence classes, Topology Appl. 18 (1984), 313-324.

11. R. Gompf and K. Miyazaki, Some well disguised ribbon knots, preprint.

12. C. Gordon, Dehn surgery and satellite knots, Trans. Amer. Math. Soc. 275 (1983), 687-708.

13. __ Ribbon concordance of knots in the 3-sphere, Math. Ann. 257 (1981), 157-170.

14. __ On primitive sets of loops in the boundary of a handlebody, Topology Appl. 27 (1987), 285-299.

15. C. Gordon and R. Litherland, On the signature of a link, Invent. Math. 47 (1978), 53-69.

16. J. Gross and T. Tucker, Topological graph theory, Wiley-Interscience, New York, 1987.

17. R. Hartley, Invertible amphicheiral knots, Math. Ann. 252 (1980), 103-109.

18. W. Jaco and P. Shalen, Seifert fibered spaces in 3-manifolds, Mem. Amer. Math. Soc. 220 (1979).

19. K. Johannson, Homotopy equivalence of 3-manifolds with boundary, Lecture Notes in Math., vol. 761, Springer-Verlag, Berlin and New York, 1979.

20. A. Kawauchi, On links not cobordant to split links, Topology 19 (1980), 321-334.

21. R. Kirby, Problems in low dimensional manifold theory, Proc. Sympos. Pure. Math., vol. 32, Amer. Math. Soc., Providence, R. I., 1978, pp. 273-312.

22. C. Livingston and P. Melvin, Algebraic knots are algebraically dependent, Proc. Amer. Math. Soc. 87 (1983), 179-180.

23. Y. Shinohara, On the signature of knots and links, Trans. Amer. Math. Soc. 156 (1971), 273-285.

24. G. Swarup, Cable knots in homotopy 3-spheres, Quart. J. Math. 31 (1980), 97-104. 
25. C. Tsau, Isomorphisms and peripheral structure of knot groups, Math. Ann. 282 (1988), 342-348.

26. F. Waldhausen, On irreducible manifolds which are sufficiently large, Ann. of Math. (2) 87 (1967), 56-88.

1-10-29 Tsujido TAIHEIDAI, Fujisawa-SHI, 251 JapaN

Current address: Faculty of Engineering, Tokyo Denki University, 2-2 Kanda-Nishikicho, Tokyo, 101 Japan

E-mail address: miyazaki@cck.dendai.ac.jp 